\title{
Counting substructures II: hypergraphs
}

\author{
Dhruv Mubayi *
}

December 23, 2012

\begin{abstract}
For various $k$-uniform hypergraphs $F$, we give tight lower bounds on the number of copies of $F$ in a $k$-uniform hypergraph with a prescribed number of vertices and edges. These are the first such results for hypergraphs, and extend earlier theorems of various authors who proved that there is one copy of $F$.

A sample result is the following: Füredi-Simonovits [11] and independently KeevashSudakov [16] settled an old conjecture of Sós [29] by proving that the maximum number of triples in an $n$ vertex triple system (for $n$ sufficiently large) that contains no copy of the Fano plane is $p(n)=\left(\begin{array}{c}\lceil n / 2\rceil \\ 2\end{array}\right)\lfloor n / 2\rfloor+\left(\begin{array}{c}\lfloor n / 2\rfloor \\ 2\end{array}\right)\lceil n / 2\rceil$. We prove that there is an absolute constant $c$ such that if $n$ is sufficiently large and $1 \leq q \leq c n^{2}$, then every $n$ vertex triple system with $p(n)+q$ edges contains at least

$$
6 q\left(\left(\begin{array}{c}
\lfloor n / 2\rfloor \\
4
\end{array}\right)+(\lceil n / 2\rceil-3)\left(\begin{array}{c}
\lfloor n / 2\rfloor \\
3
\end{array}\right)\right)
$$

copies of the Fano plane. This is sharp for $q \leq n / 2-2$.

Our proofs use the recently proved hypergraph removal lemma and stability results for the corresponding Turán problem.
\end{abstract}

*Department of Mathematics, Statistics, and Computer Science, University of Illinois, Chicago, IL 60607. email: mubayi@math.uic.edu; research supported in part by NSF grants DMS-0653946 and DMS-0969092 2000 Mathematics Subject Classification: 05A16, 05B07, 05D05 


\section{Introduction}

Mantel proved that a graph with $n$ vertices and $\left\lfloor n^{2} / 4\right\rfloor+1$ edges contains a triangle. Rademacher extended this by showing that there are at least $\lfloor n / 2\rfloor$ copies of a triangle. Subsequently, Erdös [4, 5] proved that if $q<c n$ for some small constant $c$, then $\left\lfloor n^{2} / 4\right\rfloor+q$ edges guarantees at least $q\lfloor n / 2\rfloor$ triangles. Later Lovász and Simonovits [17] proved that the same statement holds with $c=1 / 2$, thus confirming an old conjecture of Erdös. They also proved similar results for complete graphs.

In this paper (the second in a series) we initiate the study of this phenomenon to $k$-uniform hypergraphs ( $k$-graphs for short). In the first paper of this series [19], we had extended the results of Erdős and Lovász-Simonovits in two ways. First, we proved such statements for the broader class of color critical graphs. Second, we showed that all the copies of the required subgraph were incident to a small number of edges or vertices. For example, in a graph with $n$ vertices and $\left\lfloor n^{2} / 4\right\rfloor+q$ edges, $[4,5,17]$ do not give information about how the $q\lfloor n / 2\rfloor$ triangles are distributed. In [19], we proved that as long as $q=o(n)$ there are $(1-o(1)) q n / 2$ triangles incident with at most $q$ vertices.

The main new tool we have at our disposal is the recently proved hypergraph removal lemma, which is a consequence of the hypergraph regularity lemma (see Gowers [12], Nagle-RödlSchacht [23], Rödl-Skokan [27], Tao [30]). The novelty in this project is the use of the removal lemma to count substructures in hypergraphs rather precisely.

Theorem 1. (Hypergraph Removal Lemma [12, 23, 27, 30]) Fix $k \geq 2$ and a k-graph $F$ with $f$ vertices. For every $\beta>0$, there exist $\gamma>0$ and $n_{0}$ such that the following holds. Suppose that $n>n_{0}$ and an $n$ vertex $k$-graph $\mathcal{H}$ has at most $\gamma n^{f}$ copies of $F$. Then there is a set of edges in $\mathcal{H}$ of size less than $\beta n^{k}$ whose removal from $\mathcal{H}$ results in a $k$-graph with no copies of $F$.

Given a $k$-graph $F$, let ex $(n, F)$, the Turán number of $F$, be the maximum number of edges in an $n$ vertex $k$-graph with no copy of $F$. For $k>2$, determining the Turán number is a very difficult problem, and there are only sporadic results. Many of these were obtained recently by using the so-called stability approach first introduced by Erdős and Simonovits [28] in the late 1960's. Here we take this project one step further by giving asymptotically sharp results on the number of copies of a $k$-graph $F$ in a $k$-graph with $n$ vertices and $\operatorname{ex}(n, F)+q$ edges. In two cases we are able to count the exact minimum number of copies even though 
this number is quite complicated (see the abstract).

In essentially all cases where $\operatorname{ex}(n, F)$ is known, it turns out that one is guaranteed many copies of $F$ as long as there are $\operatorname{ex}(n, F)+1$ edges, so we extend all previous results that determine $\operatorname{ex}(n, F)$. It is somewhat surprising that although determining $\operatorname{ex}(n, F)$ for these hypergraphs $F$ is quite difficult (in some cases they were decades old conjectures that were only recently settled), we are able to count quite precisely the number of copies of $F$ as long as the number of extra edges $q$ is not too large. Typically we can allow $q=o\left(n^{k-1}\right)$ for the $k$-graphs we consider.

Here we should also mention the relationship between this project and recent work of Nikiforov [22] and Razborov [26] that gives asymptotically sharp estimates on the minimum number of triangles in a graph with $n$ vertices and $\left\lfloor n^{2} / 4\right\rfloor+q$ edges, where $q=\Omega\left(n^{2}\right)$. There are at present no such results for $k$-graphs for $k>2$, and little hope of achieving them. Moreover, even if such results were to be proved, they would apply only when $q=\Omega\left(n^{k}\right)$, so the results of the type $[22,26]$ will not overlap with ours. We are currently not even able to offer a conjecture about the number of copies of the $k$-graphs considered here (even the Fano plane) in this range.

Our proofs all have the following basic structure: Suppose we are given $\mathcal{H}$ with sufficiently many edges and we wish to find many copies of $F$ in $\mathcal{H}$. First we observe that if the number of copies of $F$ is very large, then we already have the bound sought. Consequently, we can use the hypergraph removal lemma to delete a small proportion of edges of $\mathcal{H}$ so that the resulting $k$-graph has no copies of $F$. Next we use the stability results that guarantee the approximate structure of $\mathcal{H}$. At this point the techniques depend highly on the particular structure of $F$ and of $\mathcal{H}$. The technical details are more involved than for the usual Turán problem, since it is not enough to find just one copy of $F$. At the end of the analysis, we are able to describe quite precisely how the copies of $F$ are distributed within $\mathcal{H}$.

We illustrate our approach on every excluded hypergraph problem for which an extremal and stability result is known. This includes many examples that have been extensively studied.

Definition 2. Let $F$ have the property that for sufficiently large n, there is a unique (up to isomorphism) k-graph $\mathcal{H}(n, F)$ with ex $(n, F)$ edges. Let $c(n, F)$ be the minimum number of copies of $F$ in the $k$-graph obtained from $\mathcal{H}(n, F)$ by adding an edge, where the minimum is taken over all possible ways to add an edge. 
Our theorems all say that if $\mathcal{H}$ is an $n$ vertex $k$-graph with $\operatorname{ex}(n, F)+q$ edges, then the number of copies of $F$ in $\mathcal{H}$ is essentially at least $q c(n, F)$. In the next subsections we will state our results precisely. We will then give a proof of the first of these results (concerning the Fano plane) and give proofs of all other results in an Appendix to this paper which can be found on the arXiv or on the author's web page.

Notation: We associate a hypergraph with its edge set. The number of edges in a hypergraph $\mathcal{H}$ is $|\mathcal{H}|$. Given hypergraphs $F, \mathcal{H}$ ( $F$ has $f$ vertices), a copy of $F$ in $\mathcal{H}$ is a subset of $f$ vertices and $|F|$ edges of $\mathcal{H}$ such that the subhypergraph formed by this set of vertices and edges is isomorphic to $F$. In other words, if we denote $A u t(F)$ to be the number of automorphisms of $F$, then the number of copies of $F$ in $\mathcal{H}$ is the number of edge-preserving injections from $V(F)$ to $V(\mathcal{H})$ divided by $A u t(F)$. For a set $S$ of vertices, define $d_{\mathcal{H}}(S)$ to be the number of edges of $\mathcal{H}$ containing $S$. If $S=\{v\}$, we simply write $d_{\mathcal{H}}(v)$. We will omit floor and ceiling symbols whenever they are not crucial, so that the presentation is clearer.

\section{Triple systems}

In this section we state our results for 3-graphs.

\subsection{Fano plane}

Let $\mathbf{F}$ be the projective plane of order two over the finite field of order two. An explicit description of $\mathbf{F}$ is $\{124,235,346,457,561,672,713\}$, obtained from the difference set $\{1,2,4\}$ over $Z_{7}$. It is well known that $\mathbf{F}$ is not 2-colorable, hence it cannot be a subgraph of any 2-colorable 3-graph. Say that a 3-graph $\mathcal{H}$ is bipartite (or 2-colorable) if it has a vertex partition $A \cup B$ such that every edge intersects both parts. Let $P^{3}(n)$ be the bipartite 3 -graph with the maximum number of edges. Note that

$$
p^{3}(n):=\left|P^{3}(n)\right|=\max _{a}\left\{\left(\begin{array}{l}
a \\
2
\end{array}\right)(n-a)+\left(\begin{array}{c}
n-a \\
2
\end{array}\right) a\right\}=(3 / 4+o(1))\left(\begin{array}{l}
n \\
3
\end{array}\right)
$$

is uniquely achieved by choosing $a \in\{\lfloor n / 2\rfloor,\lceil n / 2\rceil\}$.

Sós [29] conjectured, and Keevash-Sudakov [16] and Füredi-Simonovits [11] independently proved that among all $n$ vertex 3 -graphs ( $n$ sufficiently large) containing no copy of $\mathbf{F}$, the 
unique one with the maximum number of edges is $P^{3}(n)$. Thus $c(n, \mathbf{F})$ is defined and in fact

$$
c(n, \mathbf{F}):=6\left(\left(\begin{array}{c}
\lfloor n / 2\rfloor \\
4
\end{array}\right)+(\lceil n / 2\rceil-3)\left(\begin{array}{c}
\lfloor n / 2\rfloor \\
3
\end{array}\right)\right)=(20+o(1))(n / 4)^{4} .
$$

This is achieved by adding an edge to the part of size $\lceil n / 2\rceil$. Indeed, if we add a triple 123 to this part, then one way to make a copy of $\mathbf{F}$ is to take four points $a, b, c, d$ from the other part, partition the six pairs among $\{a, b, c, d\}$ into three perfect matchings $m_{1}, m_{2}, m_{3}$, and use the edges $\{i\} \cup p$ where $p \in m_{i}$, for each $i$ to form a copy of $\mathbf{F}$. There are $\left(\begin{array}{c}\lfloor n / 2\rfloor \\ 4\end{array}\right)$ ways to pick $a, b, c, d$ and for each such choice there are six ways to choose $m_{1}, m_{2}, m_{3}$. The only other way to form a copy of $\mathbf{F}$ using 123 is to pick four points $a, b, c, d$ with $a$ in the same part as 1 and $b, c, d$ in the other part. Then proceeding as before, we obtain $6(\lceil n / 2\rceil-3)\left(\begin{array}{c}\lfloor n / 2\rfloor \\ 3\end{array}\right)$ copies of $\mathbf{F}$. Altogether we obtain $c(n, \mathbf{F})$ copies.

Our first result shows that a 3 -graph with $p^{3}(n)+q$ edges has at least as many copies of $\mathbf{F}$ as a 3 -graph obtained from $P^{3}(n)$ by adding $q$ edges in an optimal way. The precise number we can add is

$$
q(n, \mathbf{F})= \begin{cases}n & \text { if } n \text { is even and } n / 2 \equiv 0(\bmod 4) \\ n-2 & \text { if } n \text { is even and } n / 2 \equiv 1(\bmod 4) \\ n-4 & \text { if } n \text { is even and } n / 2 \equiv 2,3(\bmod 4) \\ \lceil n / 2\rceil & \text { if } n \text { is odd and }\lceil n / 2\rceil \equiv 0(\bmod 4) \\ \lceil n / 2\rceil-1 & \text { if } n \text { is odd and }\lceil n / 2\rceil \equiv 1(\bmod 4) \\ \lceil n / 2\rceil-2 & \text { if } n \text { is odd and }\lceil n / 2\rceil \equiv 2,3(\bmod 4) .\end{cases}
$$

Theorem 3. There exists an absolute constant $\varepsilon>0$ such that if $n$ is sufficiently large and $1 \leq q \leq \varepsilon n^{2}$, then the following hold:

- Every $n$ vertex 3-graph with $p^{3}(n)+q$ edges contains at least $q c(n, \mathbf{F})$ copies of $\mathbf{F}$. This is sharp for all $q \leq q(n, \mathbf{F})$.

- If $q>q(n, \mathbf{F})$, then every $n$ vertex 3-graph with $p^{3}(n)+q$ edges contains at least $q c(n, \mathbf{F})+1$ copies of $\mathbf{F}$.

Remark. For $q>q(n, \mathbf{F})$, our proof actually gives at least $q c(n, \mathbf{F})+2\left(\begin{array}{c}\lfloor n / 2\rfloor \\ 2\end{array}\right)$ copies of $\mathbf{F}$. Theorem 3 is asymptotically sharp in a much larger range of $q$. In particular, we have the following. 
Proposition 4. For every $\varepsilon>0$ there exists $\delta>0$ and $n_{0}$ such that the following holds for all $n>n_{0}$ and $q<\delta n^{2}$. There is an $n$ vertex 3-graph with $p^{3}(n)+q$ edges and at most $(1+\varepsilon) q c(n, \mathbf{F})$ copies of $\mathbf{F}$.

\subsection{Cancellative triple-systems}

Say that a 3-graph is cancellative if whenever $A \cup B=A \cup C$ we have $B=C$. An equivalent definition is to simply say that the 3-graph does not contain a copy of two particular 3graphs: $F_{5}=\{123,124,345\}$ and $K_{4}^{3-}=\{123,124,234\}$. A 3-graph is 3-partite if it has a vertex partition into three parts such that every edge has a vertex in all three parts. Write

$$
t^{3}(n)=\left\lfloor\frac{n}{3}\right\rfloor\left\lfloor\frac{n+1}{3}\right\rfloor\left\lfloor\frac{n+2}{3}\right\rfloor
$$

for the number of edges in $T^{3}(n)$, the complete 3-partite 3-graph with the maximum number of edges. It is easy to see that $T^{3}(n)$ is cancellative.

Katona conjectured, and Bollobás [2] proved, that the maximum number of edges in an $n$ vertex cancellative 3-graph is $t^{3}(n)$, and equality holds only for $T^{3}(n)$. Later Frankl and Füredi [7] refined this by proving the same result (for $n>3000$ ) even if we just forbid $F_{5}$. Recently, Keevash and the author [14] gave a new proof of the Frankl-Füredi result while reducing the smallest $n$ value to 33 .

It is easy to see that $c\left(n, F_{5}\right)=3(n / 3)^{2}+\Theta(n)$ and this is achieved by adding a triple to $T^{3}(n)$ with two points in the largest part. In fact, even if we add a triple within one of the parts we get almost the same number of copies of $F_{5}$. Our second result shows that this is optimal, even when we are allowed to add as many as $o(n)$ edges.

Theorem 5. For every $\varepsilon>0$ there exists $\delta>0$ and $n_{0}$ such that the following holds for $n>n_{0}$. Let $\mathcal{H}$ be a 3-graph with $t^{3}(n)+q$ edges where $q<\delta n$. Then the number of copies of $F_{5}$ in $\mathcal{H}$ is at least $q(1-\varepsilon) c\left(n, F_{5}\right)$. This is asymptotically sharp for $1 \leq q<\delta n$. Moreover, if the number of copies is less than $\delta n^{3}$, then there is a collection of $q$ distinct edges that each lie in $(1-\varepsilon) c\left(n, F_{5}\right)$ copies of $F_{5}$ with no two of these edges accounting for the same copy of $F_{5}$. 


\subsection{Independent neighborhoods}

The neighborhood of a $(k-1)$-set $S$ of vertices in a $k$-graph is the set of vertices $v$ whose union with $S$ forms an edge. A set is independent if it contains no edge. We can rephrase Mantel's theorem as follows: the maximum number of edges in a 2-graph with independent neighborhoods is $\left\lfloor n^{2} / 4\right\rfloor$. This formulation can be generalized to $k>2$ and there has been quite a lot of recent activity on this question. We focus here on $k=3$, and observe that a 3 -graph has independent neighborhoods if and only if it contains no copy of $B_{5}=$ $\{123,124,125,345\}$. A 3 -graph $\mathcal{H}$ has a $(2,1)$-partition if it has a vertex partition $A \cup B$ such that $|e \cap A|=2$ for all $e \in \mathcal{H}$. Let $B^{3}(n)$ be the 3 -graph with the maximum number of edges among all those that have $n$ vertices and a $(2,1)$-partition. Note that

$$
b^{3}(n):=\left|B^{3}(n)\right|=\max _{a}\left(\begin{array}{l}
a \\
2
\end{array}\right)(n-a)=(4 / 9+o(1))\left(\begin{array}{l}
n \\
3
\end{array}\right)
$$

is achieved by choosing $a=\lfloor 2 n / 3\rfloor$ or $a=\lceil 2 n / 3\rceil$.

The author and Rödl [21] conjectured, and Füredi, Pikhurko, and Simonovits [9] proved, that among all $n$ vertex 3 -graphs ( $n$ sufficiently large) containing no copy of $B_{5}$, the unique one with the maximum number of edges is $B^{3}(n)$.

It is easy to see that $c\left(n, B_{5}\right)=2(n / 3)^{2}+\Theta(n)$ and this is achieved by adding a triple to $T^{3}(n)$ contained in the larger part. In fact, even if we add a triple within the smaller part we get almost the same number of copies of $B_{5}$. Our third result shows that this is optimal, even when we are allowed to add as many as $o\left(n^{2}\right)$ edges.

Theorem 6. For every $\varepsilon>0$ there exists $\delta>0$ and $n_{0}$ such that the following holds for $n>n_{0}$. Let $\mathcal{H}$ be a 3-graph with $b^{3}(n)+q$ edges where $q<\delta n^{2}$. Then the number of copies of $B_{5}$ in $\mathcal{H}$ is at least $q(1-\varepsilon) c\left(n, B_{5}\right)$. This is asymptotically sharp for $1 \leq q<\delta n^{2}$. Moreover, if the number of copies is less than $\delta n^{4}$, then there is a collection of $q$ distinct edges that each lie in $(1-\varepsilon) c\left(n, B_{5}\right)$ copies of $B_{5}$ with no two of these edges accounting for the same copy of $B_{5}$.

\subsection{Expanded Cliques}

Let $L_{r}$ be the 3-graph obtained from the complete graph $K_{r}$ by enlarging each edge with a new vertex. These new vertices are distinct for each edge, so $L_{r}$ has $l_{r}=r+\left(\begin{array}{c}r \\ 2\end{array}\right)=\left(\begin{array}{c}r+1 \\ 2\end{array}\right)$ 
vertices and $\left(\begin{array}{l}r \\ 2\end{array}\right)$ edges. Write $T_{r}^{3}(n)$ for the complete $r$-partite 3 -graph with the maximum number of edges. So $T_{r}^{3}(n)$ has vertex partition $V_{1} \cup \cdots \cup V_{r}$, where $n_{i}:=\left|V_{i}\right|=\lfloor(n+i-1) / r\rfloor$, and all triples with at most one point in each $V_{i}$. Define

$$
t_{r}^{3}(n):=\left|T_{r}^{3}(n)\right|=\sum_{S \in\left(\begin{array}{c}
{[r]} \\
3
\end{array}\right)} \prod_{i \in S} n_{i}
$$

Every set of $r+1$ vertices in $T_{r}^{3}(n)$ contains two vertices in the same part, and these two vertices lie in no edge. Consequently, $L_{r+1} \not \subset T_{r}^{3}(n)$.

The author [18] conjectured, and Pikhurko [24] proved, that among all $n$ vertex 3-graphs containing no copy of $L_{r+1}$ ( $r \geq 3$ fixed, $n$ sufficiently large), the unique one with the maximum number of edges is $T_{r}^{3}(n)$. Thus $c\left(n, L_{r+1}\right)$ is defined and in fact

$$
c\left(n, L_{r+1}\right)=(1+o(1))\left(\left(1-\frac{2}{r}\right) n\right)^{\left(\begin{array}{c}
r+1 \\
2
\end{array}\right)-1} \times\left(\frac{n}{r}\right)^{r-1}=\Theta\left(n^{l_{r+1}-3}\right)
$$

and this is achieved by adding a triple with exactly two points in a largest part. Our final results shows that this is asymptotically optimal, even when we are allowed to add as many as $o\left(n^{2}\right)$ edges.

Theorem 7. (Asymptotic Counting) Fix $r \geq 3$. For every $\varepsilon>0$ there exists $\delta>0$ and $n_{0}$ such that the following holds for $n>n_{0}$. Let $\mathcal{H}$ be a 3-graph with $t_{r}^{3}(n)+q$ edges where $q<\delta n^{2}$. Then the number of copies of $L_{r+1}$ in $\mathcal{H}$ is at least $q(1-\varepsilon) c\left(n, L_{r+1}\right)$. The expression $q$ is sharp for $1 \leq q<\delta n^{2}$. Moreover, if the number of copies is less than $\delta n\left(\begin{array}{c}r+1 \\ 2\end{array}\right)-1$, then there is a collection of $q$ distinct edges that each lie in $(1-\varepsilon) c\left(n, L_{r+1}\right)$ copies of $L_{r+1}$ with no two of these edges accounting for the same copy of $L_{r+1}$.

Our next result improves the asymptotic counting result above to an exact result, with a more restricted range for $q$.

Theorem 8. (Exact Counting) Fix $r \geq 3, q>0$ and let $n$ be sufficiently large. Every $n$ vertex triple system with $t_{r}^{3}(n)+q$ edges contains at least $q c\left(n, L_{r+1}\right)$ copies of $L_{r+1}$.

Theorem 8 is clearly tight, as we may add an appropriate set of $q$ pairwise disjoint edges to $T_{r}^{3}(n)$ such that each edge lies in exactly $c\left(n, L_{r+1}\right)$ copies of $L_{r+1}$. 


\section{Quadruple systems}

There are two types of 4-graphs we will be concerned with in this paper, books and expanded triangles. These represent all known cases of a 4 -graph $F$ where $\operatorname{ex}(n, F)$ has been exactly determined.

For $2 \leq l \leq 4$, the $l$-book $P_{l}$ is the 4 -graph with $l+1$ edges $l$ of which share the same three points, and another edge that contains the remaining point in each of the $l$ edges together with $4-l$ new points. Explicitly

$$
\begin{aligned}
& P_{2}=\{123 a, 123 b, a b c d\} \\
& P_{3}=\{123 a, 123 b, 123 c, a b c d\} \\
& P_{4}=\{123 a, 123 b, 123 c, 123 d, a b c d\}
\end{aligned}
$$

The expanded triangle $C_{3}$ is the 4 -graph obtained from a graph triangle by replacing each vertex by a pair of vertices. Formally,

$$
C_{3}=\{1234,3456,1256\}
$$

In the next two subsections will discuss $\mathcal{H}(n, F)$ and $c(n, F)$ for $F \in\left\{P_{2}, P_{3}, P_{4}, C_{3}\right\}$ and then we will state our results.

\subsection{Books}

$P_{2}=\{123 a, 123 b, a b c d\}$. Write

$$
\left.t^{4}(n)=\left\lfloor\frac{n}{4}\right\rfloor \frac{n+1}{4}\right\rfloor\left\lfloor\frac{n+2}{4}\right\rfloor\left\lfloor\frac{n+3}{4}\right\rfloor
$$

for the number of edges in $T^{4}(n)$, the complete 4-partite 4-graph with the maximum number of edges. It is easy to see that $T^{4}(n)$ contains no copy of $P_{2}$. Frankl and Füredi [7] conjectured, and Pikhurko [25] proved, that $\operatorname{ex}\left(n, P_{2}\right)=t^{4}(n)$ for $n$ sufficiently large. This shows that $c\left(n, P_{2}\right)$ is defined and one achieves $c\left(n, P_{2}\right)$ by adding an edge to $T^{4}(n)$ with two points in each of two parts, and no point in the remaining two parts. We then see that

$$
c\left(n, P_{2}\right)=2(n / 4)^{3}-O\left(n^{2}\right)=\Theta\left(n^{3}\right) .
$$


$P_{3}=\{123 a, 123 b, 123 c, a b c d\}$. Say that a 4-graph has a $(2,2)$-partition if it has a vertex partition into two parts so that every edge intersects each part in two points. Write

$$
d^{4}(n)=\left(\begin{array}{c}
\lfloor n / 2\rfloor \\
2
\end{array}\right)\left(\begin{array}{c}
\lceil n / 2\rceil \\
2
\end{array}\right)
$$

for the number of edges in $D^{4}(n)$, the $n$ vertex 4-graph with a $(2,2)$-partition having the maximum number of edges. It is easy to see that $D^{4}(n)$ contains no copy of $P_{3}$. Füredi, Simonovits and Pikhurko [10] proved that $\operatorname{ex}\left(n, P_{3}\right)=d^{4}(n)$ for $n$ sufficiently large. This shows that $c\left(n, P_{3}\right)$ is defined and one achieves $c\left(n, P_{2}\right)$ by adding an edge to $D^{4}(n)$ with exactly three points in the part of size $\lceil n / 2\rceil$. We then see that

$$
c\left(n, P_{3}\right)=4\left(\begin{array}{c}
\lfloor n / 2\rfloor-1 \\
2
\end{array}\right)(\lceil n / 2\rceil-3)=2(n / 2)^{3}-O\left(n^{2}\right)=\Theta\left(n^{3}\right) .
$$

$P_{4}=\{123 a, 123 b, 123 c, 123 c, a b c d\}$. A 4 -graph $\mathcal{H}$ is odd if it has a vertex partition $A \cup B$ such that every edge intersects both parts in an odd number of vertices. Let $B^{4}(n)$ be the odd 4-graph with the maximum number of edges. Note that

$$
b^{4}(n):=\left|B^{4}(n)\right|=\max _{1 \leq a \leq n}\left(\begin{array}{l}
a \\
3
\end{array}\right)(n-a)+\left(\begin{array}{c}
n-a \\
3
\end{array}\right) a
$$

is not achieved by choosing $a=\lfloor n / 2\rfloor$, but it can easily be shown that $|a-n / 2|<\sqrt{3 n} / 2+1$. Füredi, Mubayi and Pikhurko [8] proved that $\operatorname{ex}\left(n, P_{4}\right)=b^{4}(n)$ for $n$ sufficiently large. This shows that $c\left(n, P_{4}\right)$ is defined and one achieves $c\left(n, P_{2}\right)$ by adding an edge to $D^{4}(n)$ with two points in each part. We then see that

$$
c\left(n, P_{4}\right)=4\left(\begin{array}{c}
n / 2 \\
3
\end{array}\right)-O\left(n^{2}\right)=\Theta\left(n^{3}\right) .
$$

Now we state our result about counting books.

Theorem 9. Fix $l \in\{2,3,4\}$. For every $\varepsilon>0$ there exists $\delta>0$ and $n_{0}$ such that the following holds for $n>n_{0}$. Let $\mathcal{H}$ be an $n$ vertex 4-graph with ex $\left(n, P_{l}\right)+q$ edges where $q<\delta n$. Then the number of copies of $P_{l}$ in $\mathcal{H}$ is at least $q(1-\varepsilon) c\left(n, P_{l}\right)$. The expression $q$ is sharp for $1 \leq q<\delta n$. Moreover, if the number of copies is less than $\delta n^{4}$, then there is a collection of $q$ distinct edges that each lie in $(1-\varepsilon) c\left(n, P_{l}\right)$ copies of $P_{l}$ with no two of these edges accounting for the same copy of $P_{l}$. 


\subsection{Expanded triangle}

The expanded triangle is a 4-uniform example whose extremal value has been studied by Frankl [6], Sidorenko, Keevash and Sudakov [15]. Recall that $C_{3}=\{1234,3456,1256\}$ is the 4-graph obtained from a graph triangle by expanding each vertex to a set of size two. Frankl [6] proved that every $n$ vertex 4-graph containing no copy of $C_{3}$ has at most $(1+o(1)) b^{4}(n)$ edges. Recently, Keevash and Sudakov [15] sharpened this by proving that the unique 4graph that achieves this maximum is $B^{4}(n)$. Adding an edge to $B^{4}(n)$ results in at least

$$
c\left(n, C_{3}\right)=3(n / 2)^{2}-O(n)=\Theta\left(n^{2}\right)
$$

copies of $C_{3}$.

Theorem 10. For every $\varepsilon>0$ there exists $\delta>0$ and $n_{0}$ such that the following holds for $n>n_{0}$. Let $\mathcal{H}$ be an $n$ vertex 4 -graph with $b^{4}(n)+q$ edges where $q<\delta n^{2}$. Then the number of copies of $C_{3}$ in $\mathcal{H}$ is at least $q(1-\varepsilon) c\left(n, C_{3}\right)$. The expression $q$ is sharp for $1 \leq q<\delta n^{2}$. Moreover, if the number of copies is less than $\delta n^{4}$, then there is a collection of $q$ distinct edges that each lie in $(1-\varepsilon) c\left(n, C_{3}\right)$ copies of $C_{3}$ with no two of these edges accounting for the same copy of $C_{3}$.

We remark that although our proof follows the same general structure as that in [15], some new ideas are needed. In particular, since we start our proof with an application of the removal lemma, we do not have such fine control over the size of the parts in the underlying hypergraph as in [15]. Thus our approach is somewhat more robust, although the approach in [15] extends to the $k$-uniform case which we do not address here.

Throughout the paper we will frequently use the notation $\delta \ll \varepsilon$, which means that $\delta$, and any function of $\delta$ (that tends to zero with $\delta$ ) used in a proof is smaller than any function of $\varepsilon$ used in the proof. It is pretty difficult to write the precise dependence between $\delta$ and $\varepsilon$ as one of the constraints comes from an application of the removal lemma.

\section{Counting Fano's}

In this section we will prove Theorem 3 and Proposition 4. We need some lemmas about binomial coefficients. 
Lemma 11. Let $x, y, t>0$ be integers with $x+y=n, t<n^{2}$ and $s=\lceil\sqrt{2 t /(n-2)}\rceil$. Suppose that $n$ is sufficiently large and

$$
\left(\begin{array}{l}
x \\
2
\end{array}\right) y+\left(\begin{array}{l}
y \\
2
\end{array}\right) x \geq p^{3}(n)-t
$$

Then $\lfloor n / 2\rfloor-s \leq x \leq\lceil n / 2\rceil+s$ and if $t<(n-2) / 2$, then $\lfloor n / 2\rfloor-s<x<\lceil n / 2\rceil+s$.

Proof. Suppose for contradiction that $x>\lceil n / 2\rceil+s$ (the upper bound on $t$ ensures that $s<2 \sqrt{n}$ and hence $x<3 n / 4)$. Write

$$
f(x)=\left(\begin{array}{l}
x \\
2
\end{array}\right)(n-x)+\left(\begin{array}{c}
n-x \\
2
\end{array}\right) x=\frac{1}{2}(n-2) x(n-x) .
$$

Note that $p^{3}(n)=f(\lfloor n / 2\rfloor)=f(\lceil n / 2\rceil)$. Our goal therefore is to obtain the contradiction $f(x)<f(\lceil n / 2\rceil)-t$. Observe that

$$
f(a+1)=f(a)-\frac{1}{2}(n-2)(2 a+1-n) .
$$

Applying this repeatedly beginning with $a=\lceil n / 2\rceil$ we obtain

$$
\begin{aligned}
f(x)<f(\lceil n / 2\rceil+s) & =f(\lceil n / 2\rceil)-\frac{1}{2}(n-2) \sum_{a=\lceil n / 2\rceil}^{\lceil n / 2\rceil+s-1}(2 a+1-n) \\
& =f(\lceil n / 2\rceil)-\frac{1}{2} s(n-2)(s+2\lceil n / 2\rceil-n) .
\end{aligned}
$$

The choice of $s$ gives

$$
\frac{1}{2} s(n-2)(s+2\lceil n / 2\rceil-n) \geq \frac{1}{2} s^{2}(n-2) \geq t
$$

and therefore $f(x)<f(\lceil n / 2\rceil)-t$. We conclude that $x \leq\lceil n / 2\rceil+s$. Repeating this argument with $x$ replaced by $y$ gives $y \leq\lceil n / 2\rceil+s$ and hence $x \geq\lfloor n / 2\rfloor-s$.

If $t<(n-2) / 2$, and $x \geq\lceil n / 2\rceil+s$, then we only have $f(x) \leq f(\lceil n / 2\rceil+s)$. However the last inequality in (3) is strict (since $s \geq 1$ ) and we again get the same contradiction. Therefore $x<\lceil n / 2\rceil+s$ and by a similar argument, $x>\lfloor n / 2\rfloor-s$.

Recall from (1) that

$$
c(n, \mathbf{F}):=6\left(\left(\begin{array}{c}
\lfloor n / 2\rfloor \\
4
\end{array}\right)+(\lceil n / 2\rceil-3)\left(\begin{array}{c}
\lfloor n / 2\rfloor \\
3
\end{array}\right)\right) .
$$


Lemma 12. Let $x, y, s$ be positive integers with $x+y=n$ sufficiently large, $\lfloor n / 2\rfloor-s \leq$ $x \leq\lceil n / 2\rceil+s$ and $s<n / 10$. Then

$$
6\left(\begin{array}{l}
y \\
4
\end{array}\right)+6(x-3)\left(\begin{array}{l}
y \\
3
\end{array}\right) \geq c(n, \mathbf{F})-(s+3) n^{3} .
$$

Proof. Define $f(y)=6\left(\begin{array}{l}y \\ 4\end{array}\right)+6(n-y-3)\left(\begin{array}{l}y \\ 3\end{array}\right)$ and $a=\lfloor n / 2\rfloor$. Then $c(n, \mathbf{F})=f(a)$. We first observe that $f(y)$ is increasing for $1<y<n-4$. Indeed,

$$
f(y+1)-f(y)=6\left(\left(\begin{array}{l}
y \\
3
\end{array}\right)+(n-y-4)\left(\begin{array}{c}
y+1 \\
3
\end{array}\right)-(n-y-3)\left(\begin{array}{l}
y \\
3
\end{array}\right)\right)=6\left(\begin{array}{l}
y \\
2
\end{array}\right)(n-y-4)
$$

and the condition on $y$ shows that this is positive. The condition $\lfloor n / 2\rfloor-s \leq x \leq\lceil n / 2\rceil+s$ implies that $1<a-s \leq y \leq a+1+s<n-4$ and so $f(y) \geq f(a-s)$. Therefore

$$
\begin{aligned}
c(n, \mathbf{F})-f(y) & \leq f(a)-f(a-s) \\
& =6\left(\left(\begin{array}{l}
a \\
4
\end{array}\right)+(n-a-3)\left(\begin{array}{l}
a \\
3
\end{array}\right)-\left(\begin{array}{c}
a-s \\
4
\end{array}\right)-(n-a+s-3)\left(\begin{array}{c}
a-s \\
3
\end{array}\right)\right) \\
& \leq 6\left(\frac{a^{4}}{4 !}-\frac{(a-s-3)^{4}}{4 !}+(n-a-3) \frac{a^{3}}{6}-(n-a+s-3) \frac{(a-s-2)^{3}}{6}\right) \\
& \leq 6\left(\frac{a^{4}}{4 !}-\frac{a^{4}-4 a^{3} s-12 a^{3}}{4 !}+\frac{(n-a-3)}{6}\left(a^{3}-\left(a^{3}-3 a^{2} s-6 a^{2}\right)\right)\right. \\
& =6\left(\frac{4 a^{3} s+12 a^{3}}{4 !}+\frac{(n-a-3)\left(3 a^{2} s+6 a^{2}\right)}{6}\right) \\
& <a^{3} s+3 a^{3}+3 a^{2} s n+6 a^{2} n \\
& <(s+3) n^{3} .
\end{aligned}
$$

Note that (4) follows from the inequalities $(a-b)^{4}>a^{4}-4 a^{3} b$ and $(a-b)^{3}>a^{3}-3 a^{2} b$ which hold for $0<b<3 a / 2$; since $s<n / 10$ we have $0<s+2<3 a / 2-1$. This completes the proof of the Lemma.

We will need the following stability result proved independently by Keevash-Sudakov [16] and Füredi-Simonovits [11].

Theorem 13. (F Stability $[\mathbf{1 1}, \mathbf{1 6}]$ ) For every $\beta>0$, there exist $\gamma>0$ and $n_{0}$ such that the following holds. Suppose that $n>n_{0}$ and $\mathcal{H}$ is a 3-graph with $n$ vertices and $p^{3}(n)-\gamma n^{3}$ edges that contains no copy of $\mathbf{F}$. Then there is a partition of the vertex set of $\mathcal{H}$ into $X \cup Y$ so that the number of edges that are within $X$ or within $Y$ is at most $\beta n^{3}$. 
Proof of Theorem 3. Let $0<\delta \ll \varepsilon \ll 1$. Write $o_{\delta}(1)$ for any function that approaches zero as $\delta$ approaches zero and moreover, $o_{\delta}(1) \ll \varepsilon$. We emphasize that $\varepsilon$ is an absolute constant. Let $n$ be sufficiently large and let $\mathcal{H}$ be an $n$ vertex 3 -graph with $p^{3}(n)+q$ edges with $q<\varepsilon n^{2}$. Write $\# \mathbf{F}$ for the number of copies of $\mathbf{F}$ in $\mathcal{H}$.

If $\# \mathbf{F} \geq n^{6}$, then since $c(n, \mathbf{F})<n^{4}$, we have $\# \mathbf{F}>\varepsilon n^{2} c(n, \mathbf{F}) \geq q c(n, \mathbf{F})$ and we are done so assume that $\# \mathbf{F}<n^{6}=(1 / n) n^{7}$. Since $n$ is sufficiently large, by the Removal lemma there is a set of at most $\delta n^{3}$ edges of $\mathcal{H}$ whose removal results in a 3 -graph $\mathcal{H}^{\prime}$ with no copies of $\mathbf{F}$. Since $\left|\mathcal{H}^{\prime}\right|>p^{3}(n)-\delta n^{3}$, by Theorem 13, we conclude that there is a partition of $V\left(\mathcal{H}^{\prime}\right)$ (and also of $V(\mathcal{H})$ ) such that the number of edges contained entirely within a part is $o_{\delta}\left(n^{3}\right)$. Now pick a partition $X \cup Y$ of $V(\mathcal{H})$ that maximizes $e(X, Y)$, the number of edges that intersect both parts. We know that $e(X, Y) \geq p^{3}(n)-o_{\delta}\left(n^{3}\right)$, and an easy calculation also shows that each of $X, Y$ has size $n / 2 \pm o_{\delta}(n)$.

Let $B$ be the set of edges of $\mathcal{H}$ that lie entirely within $X$ or entirely within $Y$ and let $G=\mathcal{H}-B$. Let $M$ be the set of triples which intersect both parts that are not edges of $\mathcal{H}$. Then $G \cup M$ is bipartite so it has at most $p^{3}(n)$ triples. Consequently,

$$
q+|M| \leq|B| \leq o_{\delta}\left(n^{3}\right)
$$

Also, $|\mathcal{H}|=|G|+|B|$ so we may suppose that $|G|=p^{3}(n)-t$ and $|B|=q+t$ for some $t \geq 0$. For an edge $e \in B$, let $\# \mathbf{F}(e)$ be the number of copies of $\mathbf{F}$ in $\mathcal{H}$ containing the unique edge $e$ from $B$.

If $t=0$, then $G \cong P^{3}(n)$ and $\# \mathbf{F}(e) \geq c(n, \mathbf{F})$ for every $e \in B$ by (1) so we immediately obtain $\# \mathbf{F} \geq q c(n, \mathbf{F})$. If $q>q(n, \mathbf{F})$ and $\# \mathbf{F}(e)=c(n, \mathbf{F})$ for every $e \in B$, then by (2) there are two edges $e, e^{\prime} \in B$ such that $\left|e \cap e^{\prime}\right|=1$. To see this when $n$ is even, observe that if no two such edges exist, then every two edges of $B$ within $X$ intersect in zero or two points, and the same holds for the edges of $B$ within $Y$. The maximum number of edges that one can add to $P^{3}(n)$ with this property is $q(n, \mathbf{F})$, as every component is either a subset of $K_{4}^{3}$ or a sunflower with core of size two. For $n$ odd we can only have edges in the larger part and again the same argument applies.

We deduce that the number of copies of $\mathbf{F}$ containing $e$ or $e^{\prime}$ is at least $\# \mathbf{F}(e)+\# \mathbf{F}\left(e^{\prime}\right)+$ $\# \mathbf{F}\left(e, e^{\prime}\right)$ where $\# \mathbf{F}\left(e, e^{\prime}\right)$ is the number of copies of $\mathbf{F}$ in $\mathcal{H}$ containing both $e$ and $e^{\prime}$. It is easy to see that $\# \mathbf{F}\left(e, e^{\prime}\right) \geq 1$ (in fact, we have $\# \mathbf{F}\left(e, e^{\prime}\right) \geq 2\left(\begin{array}{c}\lfloor n / 2\rfloor \\ 2\end{array}\right)$ ).

We may therefore assume that $t \geq 1$ and we will now show that $\# \mathbf{F}>q c(n, \mathbf{F})$. Partition 
$B=B_{1} \cup B_{2}$, where

$$
B_{1}=\{e \in B: \# \mathbf{F}(e)>(1-\varepsilon) c(n, \mathbf{F})\}
$$

Claim 1. $\left|B_{1}\right| \geq(1-\varepsilon)|B|$

Proof of Claim. Suppose to the contrary that $\left|B_{2}\right| \geq \varepsilon|B|$. Pick $e=u v w \in B_{2}$. Write $B_{2}=B_{X X X} \cup B_{Y Y Y}$, where the subscripts have the obvious meaning (later we will use the subscript $X Y Y$ for edges with exactly two points in $Y$ ). Assume by symmetry that $e \in B_{X X X}$. For each $Y^{\prime}=\left\{y_{1}, \ldots, y_{4}\right\} \in\left(\begin{array}{c}Y \\ 4\end{array}\right)$, we can form a copy of $\mathbf{F}$ as follows: Partition the six pairs of $Y^{\prime}$ into three perfect matchings $L_{u}=\left\{e_{u}, e_{u}^{\prime}\right\}, L_{v}=\left\{e_{v}, e_{v}^{\prime}\right\}, L_{w}=\left\{e_{w}, e_{w}^{\prime}\right\}$ and for each $x \in e$, add the two triples $x \cup e_{x}$ and $x \cup e_{x}^{\prime}$. A potential copy of $\mathbf{F}$ is a copy of $\mathbf{F}$ in $G \cup M \cup B$ that uses exactly one edge of $B$. There are six ways to choose the matchings $L_{u}, L_{v}, L_{w}$, so each choice of $Y^{\prime}$ gives six potential copies of $\mathbf{F}$ containing $e$. Altogether we obtain $6\left(\begin{array}{c}|Y| \\ 4\end{array}\right)$ potential copies of $\mathbf{F}$. The only other way to form a copy of $\mathbf{F}$ using $e$ is to pick four points $a, b, c, d$ with $a \in X-e$ and $\{b, c, d\} \in\left(\begin{array}{c}Y \\ 3\end{array}\right)$. Then proceeding as before, we obtain $6(|X|-3)\left(\begin{array}{c}|Y| \\ 3\end{array}\right)$ copies of $\mathbf{F}$. This gives a total of $\left(1-o_{\delta}(1)\right) c(n, \mathbf{F})$ potential copies of $\mathbf{F}$ containing $e$. At least $(\varepsilon / 2) c(n, \mathbf{F})$ of these potential copies of $\mathbf{F}$ have a triple from $M$, for otherwise

$$
\# \mathbf{F}(e) \geq\left(1-o_{\delta}(1)-\varepsilon / 2\right) c(n, \mathbf{F})>(1-\varepsilon) c(n, \mathbf{F})
$$

which contradicts the definition of $B_{2}$. The triple from $M$ referenced above lies in at most $2\left(|X||Y|+\left(\begin{array}{c}|Y| \\ 2\end{array}\right)\right)<n^{2}$ copies of $\mathbf{F}$, so by (1), the number of triples in $M$ counted here is at least

$$
\frac{(\varepsilon / 2) c(n, \mathbf{F})}{n^{2}}>(\varepsilon / 30) n^{2} .
$$

At least a third of these triples from $M$ are incident with the same vertex of $e$, so we conclude that there exists $x \in e$ such that $d_{M}(x)>(\varepsilon / 100) n^{2}$. Let $V=X \cup Y$ and let

$$
A=\left\{v \in V: d_{M}(v)>(\varepsilon / 100) n^{2}\right\} .
$$

We have argued above that every $e \in B_{2}$ has a vertex in $A$. Consequently,

$$
3 \sum_{v \in A} d_{B_{2}}(v) \geq 3\left|B_{2}\right| \geq 3 \varepsilon|B|>3 \varepsilon|M| \geq \varepsilon \sum_{v \in A} d_{M}(v)>\varepsilon|A|(\varepsilon / 100) n^{2}
$$

and there exists a vertex $u \in A$ such that $d_{B_{2}}(u) \geq\left(\varepsilon^{2} / 300\right) n^{2}$. Assume wlog that $u \in X$ so that $d_{B_{X X X}}(u) \geq\left(\varepsilon^{2} / 300\right) n^{2}$. 
Let $\mathcal{H}_{X Y Y}$ be the set of edges in $\mathcal{H}$ with exactly one point in $X$. We may assume that $d_{H_{X Y Y}}(u) \geq d_{B_{X X X}}(u)$, for otherwise we may move $u$ to $Y$ and increase $e(X, Y)$, thereby contradicting the choice of $X, Y$. Consider

$$
e=u v w, \quad f=u y_{1} y_{2}, \quad f^{\prime}=u y_{1}^{\prime} y_{2}^{\prime}
$$

with $e \in B_{X X X}$ and $f, f^{\prime} \in \mathcal{H}_{X Y Y}, f \cap f^{\prime}=\{u\}$. The number of choices of $\left(e,\left\{f, f^{\prime}\right\}\right)$ is at least

$$
d_{B_{X X X}}(u) \times\left(\left(\begin{array}{c}
d_{\mathcal{H}_{X Y Y}}(u) \\
2
\end{array}\right)-n^{3}\right)>\varepsilon_{1} n^{6}
$$

where $\varepsilon_{1}=\varepsilon^{6} / 10^{10}$. If for at least half of the choices of $\left(e,\left\{f, f^{\prime}\right\}\right)$, these three edges span at least one copy of $\mathbf{F}$, then by $(1), \# \mathbf{F}>\left(\varepsilon_{1} / 2\right) n^{6}>q c(n, \mathbf{F})$, a contradiction. So for at least half of the choices of $\left(e,\left\{f, f^{\prime}\right\}\right)$ above, $e \cup f \cup f^{\prime}$ do not span a copy of $\mathbf{F}$. This implies that at least one of the triples $x y y^{\prime} \in M$ where $x \in e-\{u\}, y \in f-\{u\}, y^{\prime} \in f^{\prime}-\{u\}$. Since each such triple of $M$ is counted at most $|X||Y|^{2}<n^{3}$ times, we obtain the contradiction $\left(\varepsilon_{1} / 2\right) n^{6} / n^{3}<|M|=o_{\delta}\left(n^{3}\right)$. This concludes the proof of the Claim.

If $t \geq 4 \varepsilon q$, then counting copies of $\mathbf{F}$ from edges of $B_{1}$ and using Claim 1 we get

$$
\begin{aligned}
\# \mathbf{F} \geq \sum_{e \in B_{1}}(1-\varepsilon) c(n, \mathbf{F}) & \geq\left|B_{1}\right|(1-\varepsilon) c(n, \mathbf{F}) \\
& \geq(1-\varepsilon)^{2}|B| c(n, \mathbf{F}) \\
& >(1-2 \varepsilon)(q+t) c(n, \mathbf{F}) \\
& \geq\left(q+2 \varepsilon q-8 \varepsilon^{2} q\right) c(n, \mathbf{F}) \geq q c(n, \mathbf{F})
\end{aligned}
$$

and we are done. So we may assume that $t<4 \varepsilon q<4 \varepsilon^{2} n^{2}$. Let $x=|X|, y=|Y|$ and $s=\lceil\sqrt{2 t /(n-2)}\rceil$.

Claim 2. $\lfloor n / 2\rfloor-s \leq x \leq\lceil n / 2\rceil+s$ and if $t<(n-2) / 2$, then $\lfloor n / 2\rfloor-s<x<\lceil n / 2\rceil+s$.

Proof of Claim. We know that

$$
p^{3}(n)-t=|G| \leq\left(\begin{array}{l}
x \\
2
\end{array}\right) y+\left(\begin{array}{l}
y \\
2
\end{array}\right) x .
$$

Now the Claim follows immediately from Lemma 11.

Observe that $|M| \leq t$ for otherwise $|G \cup M|>p^{3}(n)$ which is impossible. Pick $e \in B$ and assume wlog that $e \subset X$. Since $t>0$, we have $1 \leq s \leq \sqrt{2 t /(n-2)}+1<n / 10$. The 
number of potential copies of $\mathbf{F}$ containing $e$, denoted $\# p \mathbf{F}(e)$, is $6\left(\begin{array}{l}y \\ 4\end{array}\right)+6(x-3)\left(\begin{array}{l}y \\ 3\end{array}\right)$. Now Claim 2, Lemma 12 and $s \geq 1$ imply that

$$
\# p \mathbf{F}(e) \geq c(n, \mathbf{F})-(s+3) n^{3} \geq c(n, \mathbf{F})-4 s n^{3} .
$$

Not all of these copies of $\mathbf{F}$ are in $\mathcal{H}$, in fact, a triple from $M$ lies in at most $2 n^{2}$ potential copies counted above (we pick either two more vertices in $Y$ or one in each of $Y$ and $X$, and there are two ways to complete a potential copy of $\mathbf{F}$ containing $e$ ). We conclude that

$$
\# \mathbf{F}(e) \geq \# p \mathbf{F}(e)-2 n^{2}|M| \geq c(n, \mathbf{F})-4 s n^{3}-2 n^{2}|M| \geq c(n, \mathbf{F})-4 s n^{3}-2 t n^{2}
$$

Suppose first that $t<(n-2) / 2$. Then Claim 2 gives $\lfloor n / 2\rfloor-s<x<\lceil n / 2\rceil+s$. Since $s=1$ and $x$ is an integer, $|x-n / 2|<1$. By $(1)$,

$$
\# p \mathbf{F}(e) \geq \min \left\{6\left(\begin{array}{l}
y \\
4
\end{array}\right)+6(x-3)\left(\begin{array}{l}
y \\
3
\end{array}\right): x \in\{\lfloor n / 2\rfloor,\lceil n / 2\rceil\}\right\} \geq c(n, \mathbf{F}) .
$$

Consequently, we can refine the bound in (5) to

$$
\# \mathbf{F}(e) \geq c(n, \mathbf{F})-2 t n^{2}
$$

Altogether,

$$
\# \mathbf{F} \geq \sum_{e \in B} \# \mathbf{F}(e) \geq(q+t)\left(c(n, \mathbf{F})-2 t n^{2}\right)=q c(n, \mathbf{F})+t c(n, \mathbf{F})-2 q t n^{2}-2 t^{2} n^{2} .
$$

Let us recall that $q \leq \varepsilon n^{2}$ and $0<t<4 \varepsilon q$. Then by (1), $2 q t n^{2}<2 \varepsilon t n^{4}<(t / 2) c(n, \mathbf{F})$ and $2 t^{2} n^{2}=2 t\left(t n^{2}\right)<(8 \varepsilon q) t n^{2}<8 \varepsilon^{2} t n^{4}<(t / 2) c(n, \mathbf{F})$. Consequently, \#F $>q c(n, \mathbf{F})$ as required.

Next we suppose that $t \geq(n-2) / 2>n / 4$. This implies that $s \leq \sqrt{2 t /(n-2)}+1 \leq 4 \sqrt{t / n}$ and $\sqrt{t} \leq 2 t / \sqrt{n}$. Therefore

$$
4 q s n^{3}<16 q n^{3} \sqrt{t / n}=16 q \sqrt{t} n^{2.5} \leq 32 q t n^{2} \leq 32 \varepsilon t n^{4}<(t / 5) c(n, \mathbf{F}) .
$$

So we again use (5) to deduce that $\# \mathbf{F}$ is at least

$\sum_{e \in B} \# \mathbf{F}(e) \geq(q+t)\left(c(n, \mathbf{F})-4 s n^{3}-2 t n^{2}\right) \geq q c(n, \mathbf{F})+t c(n, \mathbf{F})-4 q s n^{3}-2 q t n^{2}-4 t s n^{3}-2 t^{2} n^{2}$

As $t<q<\varepsilon n^{2}$ we have, using (1), the bounds

$$
2 q t n^{2}<(t / 5) c(n, \mathbf{F}), \quad 4 t s n^{3}<4 q s n^{3}<(t / 5) c(n, \mathbf{F}), \quad 2 t^{2} n^{2}<(t / 5) c(n, \mathbf{F}) .
$$


This shows that $\# \mathbf{F}>q c(n, \mathbf{F})$ as required.

To see that the result is tight for $q \leq q(n, \mathbf{F})$ observe that we may add $q$ edges to $P^{3}(n)$ with every two edges sharing zero or two points. If $n$ is even, we do this by adding to each part of $P^{3}(n)$ the maximum number of edge that pairwise share zero or two points. This is achieved by adding disjoint copies of $K_{4}^{3}$, the complete 3-graph on four points, or collections of edges that pairwise share the same two points. If $n$ is odd, then we add edges only to the larger part. Each added edge lies in exactly $c(n, \mathbf{F})$ copies of $\mathbf{F}$ and no two added edges lie in any copy of $\mathbf{F}$. So the total number of copies of $\mathbf{F}$ is exactly $q c(n, \mathbf{F})$. This completes the proof of the theorem.

We end this section by proving that this result is asymptotically sharp.

Proof of Proposition 4. Let $0<\delta \ll \varepsilon$. Consider the following construction: Add a collection of $q$ edges to $P^{3}(n)$ within the part of size $\lceil n / 2\rceil$ such that the following two conditions hold.

(1) every two added edges have at most one point in common and

(2) the added edges do not form a Pasch configuration, which is the six vertex 3-graph obtained from $\mathbf{F}$ by deleting a vertex.

It is well-known that such triple systems exist of size $\delta n^{2}$ (in fact such Steiner triple systems also exist [13]). Each new edge lies in at most $c(n, \mathbf{F})$ copies of $\mathbf{F}$ that contain a unique new edge. Now suppose that two of these new edges, say $e, e^{\prime}$ lie in a copy $C$ of $\mathbf{F}$. Then there are at most $n^{2}$ choices for the remaining two vertices of $C$. So the number of copies of $\mathbf{F}$ containing two new edges is at most $q^{2} n^{2} \leq \delta q n^{4}<\varepsilon q c(n, \mathbf{F})$. There are no copies of $\mathbf{F}$ using three new edges since three edges of $\mathbf{F}$ either span seven vertices or form a Pasch configuration. In either case we would have a Pasch configuration among the added edges. Consequently, the number of copies of $\mathbf{F}$ is at most $q(1+\varepsilon) c(n, \mathbf{F})$.

\section{Concluding Remarks}

- We have given counting results for every $k$-graph for which a stability result is known except for one family which is derived from the expanded cliques. This was studied in [20], and included the triple system $\{123,145,167,357\}$ which is the smallest non-3-partite linear 
(every two edges share at most one vertex) 3-graph. It appears that our approach will give appropriate counting results for this problem as well and we did not feel motivated to carry out the details.

- Our results suggest that whenever one can obtain stability and exact results for an extremal problem, one can also obtain counting results. However, in each case the argument is different. It would be interesting to unify this approach (at least for certain classes) so one does not have to use new methods for each $F$. We formulate this as a conjecture. Say that a $k$-graph $F$ is stable if $\operatorname{ex}(n, F)$ is achieved uniquely by the $n$ vertex 3 -graph $\mathcal{H}(n)$ for sufficiently large $n$, and every $n$ vertex 3 -graph with $(1-o(1)) \operatorname{ex}(n, F)$ edges and no copy of $F$ can be obtained from $\mathcal{H}(n)$ by changing at most $o\left(n^{3}\right)$ edges.

Conjecture 14. Let $F$ be a non $k$-partite stable $k$-graph. For every positive integer $q$, the following holds for sufficiently large $n$ : Every $n$ vertex $k$-graph with ex $(n, F)+q$ edges contains at least $q c(n, F)$ copies of $F$.

- We have not been able to prove exact counting results for most $k$-graphs we consider. The reason for this is that we need to use the minimum degree condition in the proofs and we don't know how to get around this technical difficulty.

- All our theorems find $\alpha(1-o(1)) n^{\beta}$ copies of $F$ on an edge, or $\delta n^{\gamma}$ copies of $F$ altogether, for suitable $\alpha, \beta, \gamma, \delta$. However, in each case our proofs give $\delta n^{\gamma}$ copies of $F$ on a single vertex.

- Our results for $F_{5}$ (among 3-graphs) appear to be weaker than the other results. In particular, we only allow $q<\delta n$ unlike in the other cases where we allow $q<\delta n^{2}$. However, using our approach this cannot be improved further. Indeed, for any $\varepsilon>0$ (take $\varepsilon=1 / 2$ for example) and all $n$, there exists an $n$ vertex 3 -graph $\mathcal{H}$ with $t^{3}(n)+\varepsilon n$ edges and the following two properties:

(1) for every edge $e \in \mathcal{H}$, the number of copies of $F_{5}$ containing $e$ is less than $(3-\varepsilon)(n / 3)^{2}$

(2) the number of copies of $F_{5}$ in $\mathcal{H}$ is less then $\varepsilon n^{3}$.

To see this, let $T^{3}(n)$ have parts $X, Y, Z$ and construct $\mathcal{H}$ as follows. Pick $(x, y) \in X \times Y$, delete $\varepsilon n / 3$ edges of the form $x y z$ with $z \in Z$, and add $4 \varepsilon n / 3$ edges of the form $x_{i} x y$ with $x_{i} \in X$. Then $|\mathcal{H}|=t^{3}(n)+\varepsilon n$. A copy of $F_{5}$ in $\mathcal{H}$ must contain an edge $e_{i}=x_{i} x y$, and the number of copies containing $e_{i}$ is at most $(3-\varepsilon)(n / 3)^{2}$. Therefore the total number of copies of $F_{5}$ in $\mathcal{H}$ is at most $(4 \varepsilon n / 3)(3-\varepsilon)(n / 3)^{2}<\varepsilon n^{3}$. 
- Our results for $L_{r}$ can be extended to the $k$-uniform case without too much difficulty. We describe some of the details below. For $r>k \geq 2$, Let $L_{r}^{k}$ be the $k$-graph obtained from the complete graph $K_{r}$ by enlarging each edge with a set of $k-2$ new vertices. These sets of new vertices are disjoint for each edge, so $L_{r}^{k}$ has $r+(k-2)\left(\begin{array}{l}r \\ 2\end{array}\right)$ vertices and $\left(\begin{array}{l}r \\ 2\end{array}\right)$ edges. Write $T_{r}^{k}(n)$ for the complete $r$-partite $k$-graph with the maximum number of edges. So $T_{r}^{k}(n)$ has vertex partition $V_{1} \cup \cdots \cup V_{r}$, where $n_{i}:=\left|V_{i}\right|=\lfloor(n+i-1) / r\rfloor$, and all $k$-sets with at most one point in each $V_{i}$. Define

$$
t_{r}^{k}(n):=\left|T_{r}^{k}(n)\right|=\sum_{S \in\left(\begin{array}{c}
{[r]} \\
k
\end{array}\right)} \prod_{i \in S} n_{i} .
$$

Every set of $r+1$ vertices in $T_{r}^{k}(n)$ contains two vertices in the same part, and these two vertices lie in no edge. Consequently, $L_{r+1}^{k} \not \subset T_{r}^{k}(n)$. The author [18] conjectured, and Pikhurko [24] proved, that among all $n$ vertex $k$-graphs containing no copy of $L_{r+1}^{k}$ ( $r \geq k \geq 2$ fixed, $n$ sufficiently large), the unique one with the maximum number of edges is $T_{r}^{k}(n)$. Define $c_{r+1}^{k}(n)$ to be the minimum number of copies of $L_{r+1}^{k}$ in a $k$-graph obtained from $T_{r}^{k}(n)$ by adding one edge. The following theorem can be proved by extending the ideas of [24] and Theorem 7's proof in the obvious way.

Theorem 15. Fix $r \geq k \geq 3$. For every $\varepsilon>0$ there exists $\delta>0$ and $n_{0}$ such that the following holds for $n>n_{0}$. Let $\mathcal{H}$ be a k-graph with $t_{r}^{k}(n)+q$ edges where $q<\delta n^{k-1}$. Then the number of copies of $L_{r+1}^{k}$ in $\mathcal{H}$ is at least $q(1-\varepsilon) c_{r+1}^{k}(n)$. The expression $q$ is sharp for

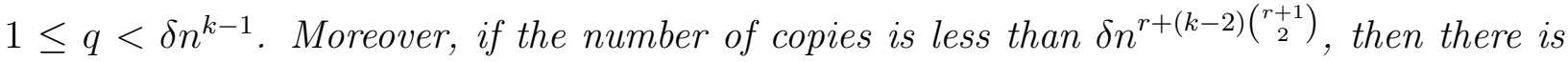
a collection of $q$ distinct edges that each lie in $(1-\varepsilon) c_{r+1}^{k}(n)$ copies of $L_{r+1}^{k}$ with no two of these edges accounting for the same copy of $L_{r+1}^{k}$.

The exact result for this situation can also be proved using the same methods.

Alon and Pikhurko [1] proved that $\operatorname{ex}\left(n, L^{k}(G)\right)=t_{r}^{k}(n)$ (for $n>n_{0}$ ) where $L^{k}(G)$ is the $k$-graph obtained from an $r$-color critical graph $G$ by expanding each edge of $G$ by a new set of $k-2$ vertices. In [14] we had proved the corresponding counting result for $L^{2}(G)$ and those ideas combined with the ones in this paper can be used to give similar results for $L^{k}(G)$. 


\section{Acknowledgments}

The author is very grateful to the referees for their careful reading and helpful comments.

\section{References}

[1] N. Alon, O. Pikhurko, personal communication (2007)

[2] B. Bollobás, Three-graphs without two triples whose symmetric difference is contained in a third, Discrete Math, 8 (1974) 21-24.

[3] D. de Caen, Z. Füredi, The maximum size of 3-uniform hypergraphs not containing a Fano plane. J. Combin. Theory Ser. B 78 (2000), no. 2, 274-276.

[4] P. Erdős, On a theorem of Rademacher-Turán, Illinois Journal of Math, 6, (1962), $122-127$

[5] P. Erdős, On the number of complete subgraphs contained in certain graphs, Magy. Tud. Acad. Mat. Kut. Int. Közl. 7, (1962), 459-474

[6] P. Frankl, Asymptotic solution of a Turán-type problem, Graphs Combin. 6 (1990), no. $3,223-227$.

[7] P. Frankl, Z. Füredi, A new generalization of the Erdös-Ko-Rado theorem, Combinatorica 3 (1983), no. 3-4, 341-349.

[8] Z. Füredi, D. Mubayi, O. Pikhurko, Quadruple systems with independent neighborhoods, J. Combin. Theory Ser. A 115 (2008), no. 8,

[9] Z. Füredi, O. Pikhurko, M. Simonovits, On triple systems with independent neighbourhoods, Combin. Probab. Comput. 14 (2005), no. 5-6, 795-813.

[10] Z. Füredi, O. Pikhurko, M. Simonovits, 4-Books of Three Pages, J Combin Theory Ser. A, 113 (2006) 882-891.

[11] Z. Füredi, M. Simonovits, Triple systems not containing a Fano configuration, Combin. Probab. Comput. 14 (2005), no. 4, 467-484. 
[12] W. T. Gowers, Hypergraph regularity and the multidimensional Szemerdi theorem, Ann. of Math. (2) 166 (2007), no. 3, 897-946.

[13] M. J. Grannell, T. S. Griggs, C. A. Whitehead, The resolution of the anti-Pasch conjecture, J. Combin. Des. 8 (2000), no. 4, 300-309.

[14] P. Keevash, D. Mubayi, Stability theorems for cancellative hypergraphs, J. Combin. Theory Ser. B 92 (2004), no. 1, 163-175.

[15] P. Keevash, B. Sudakov, On a hypergraph Turán problem of Frankl, Combinatorica (25) $62005,673-706$

[16] P. Keevash, B. Sudakov, The Turán number of the Fano plane, Combinatorica 25 (2005), no. $5,561-574$.

[17] L. Lovász, M. Simonovits, On the number of complete subgraphs of a graph. II, Studies in pure mathematics, 459-495, Birkhuser, Basel, 1983.

[18] D. Mubayi, A hypergraph extension of Turán's theorem, J. Combin. Theory Ser. B 96 (2006), no. 1, 122-134.

[19] D. Mubayi, Counting substructures I: color critical graphs, Advances in Mathematics, to appear (available at http://www.math.uic.edu/ mubayi/papers/graphcount.pdf)

[20] D. Mubayi, O. Pikhurko, A new generalization of Mantel's theorem to $k$-graphs, Journal of Combinatorial Theory, Series B, 97 (2007), no. 4, 669-678

[21] D. Mubayi, V. Rödl, On the Turán number of triple systems, J. of Combin. Theory, Ser. A, 100 (2002), no. 1, 136-152

[22] V. Nikiforov, The number of cliques in graphs of given order and size, submitted

[23] B. Nagle, V. Rödl, M. Schacht, The counting lemma for regular $k$-uniform hypergraphs, Random Structures Algorithms 28 (2006), no. 2, 113-179.

[24] O. Pikhurko, Exact Computation of the Hypergraph Turan Function for Expanded Complete 2-Graphs, 9pp, accepted by J. Combin. Theory, Ser. B, publication suspended for an indefinite time, see http://www.math.cmu.edu/ pikhurko/Copyright.html 
[25] O. Pikhurko, An exact Turn result for the generalized triangle. Combinatorica 28 (2008), no. $2,187-208$.

[26] A. Razborov, On the Minimal Density of Triangles in Graphs, Combinatorics, Probability and Computing, Vol. 17, No 4, 2008, 603-618.

[27] V. Rödl, J. Skokan, Applications of the regularity lemma for uniform hypergraphs, Random Structures Algorithms 28 (2006), no. 2, 180-194.

[28] M. Simonovits, A method for solving extremal problems in graph theory, stability problems, 1968 Theory of Graphs (Proc. Colloq., Tihany, 1966) pp. 279-319 Academic Press, New York

[29] V. Sós, Remarks on the connection of graph theory, finite geometry and block designs; in: Teorie Combinatorie, Tomo II, Accad. Naz. Lincei, Rome, 1976, 223-233.

[30] T. Tao, A variant of the hypergraph removal lemma, J. Combin. Theory Ser. A 113 (2006), no. 7, 1257-1280.

\section{Appendix}

\subsection{Counting $F_{5}$ 's}

Theorem 5 follows from the following result. Recall that $c\left(n, F_{5}\right)=(3+o(1))(n / 3)^{2}$.

Theorem 16. For every $\varepsilon>0$ there exists $\delta>0$ and $n_{0}$ such that the following holds for $n>n_{0}$. Every $n$-vertex 3-graph with $t^{3}(n)+1$ edges contains either

- an edge that lies in at least $(3-\varepsilon)(n / 3)^{2}$ copies of $F_{5}$, or

- at least $\delta n^{3}$ copies of $F_{5}$.

Proof of Theorem 5. Remove $q-1$ edges from $\mathcal{H}$ and apply Theorem 16. If we find $\delta n^{3}$ copies of $F_{5}$, then since $q<\delta n$, the number of copies is much larger than $q(1-\varepsilon) c\left(n, F_{5}\right)$ and we are done. Consequently, we find an edge $e_{1}$ in at least $(3-\varepsilon)(n / 3)^{2}>(1-\varepsilon) c\left(n, F_{5}\right)$ copies of $F_{5}$. Now remove $q-2$ edges from $\mathcal{H}-e_{1}$ and repeat this argument to obtain $e_{2}$. In this way we obtain edges $e_{1}, \ldots, e_{q}$ as required. 
Sharpness follows by adding a 3-partite triple system to one of the parts of $T^{3}(n)$. It is easy to see that each added edge lies in $c\left(n, F_{5}\right)-O(1)$ copies of $F_{5}$ and no copy of $F_{5}$ contains two of the new edges. Consequently, the copies of $F_{5}$ are counted exactly once.

We will need the following stability theorem for $F_{5}$ proved by Keevash and the first author [14].

Theorem 17. ( $F_{5}$ Stability [14]) Let $\mathcal{H}$ be a 3-graph with $n$ vertices and $t^{3}(n)-o\left(n^{3}\right)$ edges that contains no copy of $F_{5}$. Then there is a partition of the vertex set of $\mathcal{H}$ into three parts so that the number of edges with at least two vertices in some part is o( $\left.n^{3}\right)$. In other words, $\mathcal{H}$ can be obtained from $T^{3}(n)$ by adding and deleting a set of o( $\left.n^{3}\right)$ edges.

Proof of Theorem 16. Given $\varepsilon$ let $0<\delta \ll \varepsilon$. Write $o_{\delta}(1)$ for a function that approaches zero as $\delta$ approaches zero and moreover, $o_{\delta}(1) \ll \varepsilon$ for the set of functions used in this proof. Let $n$ be sufficiently large and let $\mathcal{H}$ be an $n$ vertex 3 -graph with $t^{3}(n)+1$ edges. Write $\# F_{5}$ for the number of copies of $F_{5}$ in $\mathcal{H}$.

We first argue that we may assume that $\mathcal{H}$ has minimum degree at least $d=(2 / 9)\left(1-\delta_{1}\right)\left(\begin{array}{l}n \\ 2\end{array}\right)$, where $\delta_{1}=\delta^{1 / 4}$. Indeed, if this is not the case, then remove a vertex of degree less than $d$ to form the 3 -graph $\mathcal{H}_{1}$ with $n-1$ vertices. Continue removing a vertex of degree less than $d$ if such a vertex exists. If we could continue this process for $\delta_{2} n$ steps, where $\delta_{2}=\delta^{1 / 2}$, then the resulting 3 -graph $\mathcal{H}^{\prime}$ has $\left(1-\delta_{2}\right) n$ vertices and number of edges at least

$$
\begin{aligned}
\frac{2}{9}(1-\delta / 2)\left(\begin{array}{l}
n \\
3
\end{array}\right)-\left(\delta_{2} n\right) \frac{2}{9}\left(1-\delta_{1}\right)\left(\begin{array}{l}
n \\
2
\end{array}\right) & \geq \frac{2}{9}\left(1-\delta-3 \delta_{2}\left(1-\delta_{1}\right)\right)\left(\begin{array}{l}
n \\
3
\end{array}\right) \\
& >\frac{2}{9}(1+\delta)\left(1-\delta_{2}\right)^{3}\left(\begin{array}{l}
n \\
3
\end{array}\right) \\
& >\frac{2}{9}(1+\delta)\left(\begin{array}{c}
\left(1-\delta_{2}\right) n \\
3
\end{array}\right) .
\end{aligned}
$$

By the result of Keevash-Mubayi [14] and Erdős-Simonovits supersaturation we conclude that $\mathcal{H}$ has at least $\delta^{\prime} n^{5}$ copies of $F^{5}$ (for some fixed $\delta^{\prime}>0$ ) and we are done. So we may assume that this process of removing vertices of degree less than $d$ terminates in fewer than $\delta_{2} n$ steps, and when it terminates we are left with a 3 -graph $\mathcal{H}^{\prime}$ on $n^{\prime}>\left(1-\delta_{2}\right) n$ vertices and minimum degree at least $d$.

Now suppose that we could prove that there is an edge of $\mathcal{H}^{\prime}$ that lies in at least $(3-$ $\varepsilon / 2)\left(n^{\prime} / 3\right)^{2}$ copies of $F_{5}$. Since $\delta \ll \varepsilon$, this is greater than $(3-\varepsilon)(n / 3)^{2}$ and we are done. If 
on the other hand $\mathcal{H}^{\prime}$ contains at least $2 \delta n^{\prime 3}$ copies of $F_{5}$, then again this is at least $\delta n^{3}$ and we are done. So if we could prove the result for $\mathcal{H}^{\prime}$ with $2 \delta, \varepsilon / 2$, then we could prove the result for $\mathcal{H}$ (with $\delta, \varepsilon$ ). Consequently, we may assume that $\mathcal{H}$ has minimum degree at least $\left(2 / 9-o_{\delta}(1)\right)\left(\begin{array}{l}n \\ 2\end{array}\right)=\left(1-o_{\delta}(1)\right)(n / 3)^{2}$.

If $\# F_{5} \geq \delta n^{5}$, then we are done so assume that $\# F_{5}<\delta n^{4}$. Then by the Removal lemma, there is a set of at most $o_{\delta}\left(n^{3}\right)$ edges of $\mathcal{H}$ whose removal results in a 3-graph $\mathcal{H}^{\prime}$ with no copies of $F_{5}$. Since $\left|\mathcal{H}^{\prime}\right|>t^{3}(n)-o_{\delta}\left(n^{3}\right)$, by Theorem 17 , we conclude that there is a 3 -partition of $\mathcal{H}^{\prime}$ (and also of $\mathcal{H}$ ) such that the number of edges with at least two points in a part is $o_{\delta}\left(n^{3}\right)$. Now pick a partition $X \cup Y \cup Z$ of $\mathcal{H}$ that maximizes $e(X, Y, Z)=\mathcal{H} \cap(X \times Y \times Z)$. We know that $e(X, Y, Z) \geq t^{3}(n)-o_{\delta}\left(n^{3}\right)$, and an easy calculation also shows that each of $X, Y, Z$ has size $n / 3+o_{\delta}(n)$.

Let $B=\mathcal{H}-(X \times Y \times Z)$ be the set of edges of $\mathcal{H}$ that have at least two points in one of the partition classes and set $G=\mathcal{H}-B$. Let $M=(X \times Y \times Z)-\mathcal{H}$ be the set of triples with one point in each of $X, Y, Z$ that are not edges of $\mathcal{H}$. Then $G \cup M=(\mathcal{H}-B) \cup M$ is 3 -partite so it has at most $t^{3}(n)$ triples. Since $|\mathcal{H}|=t^{3}(n)+1$, we conclude that

$$
0 \leq|M|<|B|=o_{\delta}\left(n^{3}\right)
$$

Claim. For every vertex $v$ of $\mathcal{H}$ we have $d_{M}(v)<\varepsilon^{\prime}(n / 3)^{2}$ for $\varepsilon^{\prime}=\varepsilon / 10^{6}$.

Proof of Claim. Suppose for contradiction that $d_{M}(v) \geq \varepsilon^{\prime}(n / 3)^{2}$ for some vertex $v$. Then

$$
\left(1-o_{\delta}(1)\right)(n / 3)^{2} \leq d_{\mathcal{H}}(v)=d_{G}(v)+d_{B}(v) \leq\left(1+o_{\delta}(1)\right)(n / 3)^{2}-\varepsilon^{\prime}(n / 3)^{2}+d_{B}(v) .
$$

We conclude that $d_{B}(v) \geq\left(\varepsilon^{\prime}-o_{\delta}(1)\right)(n / 3)^{2}>\left(\varepsilon^{\prime} / 2\right)(n / 3)^{2}$. Assume wlog that $v \in X$.

Case 1: $d_{B_{X X X}}(v)>\left(\varepsilon^{\prime} / 10\right)(n / 3)^{2}$. Suppose that $e=u v w$ satisfies $v \in e \in B_{X X X}$ and $(y, z) \in Y \times Z$. The number of such choices for $(e,(y, z))$ is at least $d_{B_{X X X}}(v)|Y||Z|>$ $\left(\varepsilon^{\prime} / 20\right)(n / 3)^{4}$. If for at least half of these choices $e \cup\{y, z\}$ forms a copy of $F_{5}$ via the edges $e, u y z$, wyz then we have $\# F_{5}>\left(\varepsilon^{\prime} / 40\right)(n / 3)^{4}>\delta n^{3}$, a contradiction. So for at least half of the choices of $(e,(y, z))$ above, $x y z \notin \mathcal{H}$ for some $x \in\{u, w\}$ (i.e. $x y z \in M)$. Since each such triple of $M$ is counted at most $|X|<n$ times (as $v$ is fixed), we obtain the contradiction $\left(\varepsilon^{\prime} / 40 n\right)(n / 3)^{4}<|M|=o_{\delta}\left(n^{3}\right)$. This concludes the proof in this case.

Case 2: $d_{B_{X X Y}}(v)>\left(\varepsilon^{\prime} / 10\right)(n / 3)^{2}$ or $d_{B_{X X Z}}(v)>\left(\varepsilon^{\prime} / 10\right)(n / 3)^{2}$. Assume by symmetry that $d_{B_{X X Y}}(v)>\left(\varepsilon^{\prime} / 10\right)(n / 3)^{2}$. We may assume that $d_{G}(v) \geq d_{B_{X X Y}}(v)$ for otherwise we can 
move $v$ to $Z$ and contradict the choice of the partition. Suppose that $e=u v w$ satisfies $v \in e \in B_{X X Y}$ with $u \in X, w \in Y$. Let $(y, z) \in(Y-\{w\}) \times Z$ be such that $v y z \in \mathcal{H}$. The number of such choices for $(e,(y, z))$ is at least $d_{B_{X X Y}}(v)\left(d_{G}(v)-|Z|\right)>\left(\varepsilon^{\prime} / 11\right)^{2}(n / 3)^{4}$. If for at least half of these choices $e \cup\{y, z\}$ forms a copy of $F_{5}$ via the triples $e, u y z, v y z$ then we have $\# F_{5}>\left(\varepsilon^{\prime} / 20\right)^{2}(n / 3)^{4}>\delta n^{3}$, a contradiction. So for at least half of the choices of $(e,(y, z))$ above, uyz $\notin \mathcal{H}$ (i.e. uyz $\in M)$. Since each such triple of $M$ is counted at most $|Y|<n$ times (as $v$ is fixed), we obtain the contradiction $\left(\varepsilon^{\prime} / 20\right)^{2}(n / 3)^{4} / n<|M|=o_{\delta}\left(n^{3}\right)$. This concludes the proof in this case.

Case 3: $\quad d_{B_{X Y Y}}(v)>\left(\varepsilon^{\prime} / 10\right)(n / 3)^{2}$ or $d_{B_{X Z Z}}(v)>\left(\varepsilon^{\prime} / 10\right)(n / 3)^{2}$. Assume by symmetry that $d_{B_{X Y Y}}(v)>\left(\varepsilon^{\prime} / 10\right)(n / 3)^{2}$. Suppose that $e=u v w$ satisfies $v \in e \in B_{X X Y}$ with $u, w \in Y$. Pick $(x, z) \in(X-\{v\}) \times Z$. The number of such choices for $(e,(x, z))$ is at least $d_{B_{X Y Y}}(v)(|X|-1)|Z|>\left(\varepsilon^{\prime} / 11\right)^{2}(n / 3)^{4}$. If for at least half of these choices $e \cup\{x, z\}$ forms a copy of $F_{5}$ via the triples $x z u, x z w, e$ then we have $\# F_{5}>\left(\varepsilon^{\prime} / 20\right)^{2}(n / 3)^{4}>\delta n^{3}$, a contradiction. So for at least $\left(\varepsilon^{\prime} / 20\right)^{2}(n / 3)^{4}$ of the choices of $(e,(x, z))$ above, $x y z \notin \mathcal{H}$ for some $y \in\{u, w\}$ (i.e. $x y z \in M$ ). For at least half of these choices, we may assume that $y=u$. Since each such triple of $M$ is counted at most $|Y|<n$ times (as $v$ is fixed), we obtain the contradiction $\left(\varepsilon^{\prime} / 20\right)^{2}(n / 3)^{4} / 2 n<|M|=o_{\delta}\left(n^{3}\right)$. This concludes the proof of the Claim.

Let $B_{1}=B_{X X X} \cup B_{Y Y Y} \cup B_{Z Z Z} \subset B$, where the subscripts have the obvious meaning ( $B_{X X X}$ is the set of edges in $B$ with three points in $X$ etc.), and let $B_{2}=B-B_{1}$, so $B_{2}$ consists of those edges of $\mathcal{H}$ that have two points in one part and one point in some other part.

Suppose that $e=u v w \in B_{X X X}$. For each $(y, z) \in Y \times Z$ the points $u, v, w, y, z$ form a potential copy of $F_{5}$ via $e$ and two triples involving $y, z$. For at least $(\varepsilon / 2)(n / 3)^{2}$ of these potential copies, $x y z \in M$ for $x \in e$, otherwise $e$ lies in $\left(3-o_{\delta}(1)-\varepsilon / 2\right)(n / 3)^{2}>(3-\varepsilon)(n / 3)^{2}$ copies of $F_{5}$ and we are done. Each such triple of $M$ is counted at most twice, hence the number of triples intersecting $e$ is at least $(\varepsilon / 4)(n / 3)^{2}$, and at least a third of these triples contain the same vertex $x \in e$. We conclude that $d_{M}(x)>(\varepsilon / 12)(n / 3)^{3} \geq \varepsilon^{\prime}(n / 3)^{2}$ which contradicts the Claim. The argument above works for any $e \in B_{1}$, so we have shown that $B_{1}=\emptyset$.

Let $e=u v w \in B_{2}=B$, where $u, v$ are in the same part, say $X$, and $w$ is in another part, say $Y$. For each $(y, z) \in(Y-\{w\}) \times Z$, there are three types of potential copies of $F_{5}$ with vertices $u, v, w, y, z$ : 
Type 1: uyz,vyz,e

Type 2: uwz,e,vyz or vwz,e,uyz

The number of Type $i$ potential copies of $F_{5}$ is $(|Y|-1)|Z|=\left(1-o_{\delta}(1)\right)(n / 3)^{2}$. We may assume that the number of Type 1 (real, not potential) copies of $F_{5}$ is at most $(1-\varepsilon / 3)(n / 3)^{2}$, or that the number of Type 2 (real, not potential) copies of $F_{5}$ is at most $(2-2 \varepsilon / 3)(n / 3)^{2}$. Otherwise $e$ lies in at least $(3-\varepsilon)(n / 3)^{2}$ copies of $F_{5}$ and we are done.

Suppose that the number of Type 1 copies of $F_{5}$ is at most $(1-\varepsilon / 3)(n / 3)^{2}$. The number of pairs $(y, z) \in(Y-\{w\}) \times Z$ for which either $u y z \in M$ or $v y z \in M$ is at least

$$
(|Y|-1)|Z|-(1-\varepsilon / 3)(n / 3)^{2}>\left(1-o_{\delta}(1)-1+\varepsilon / 3\right)(n / 3)^{2}>(\varepsilon / 4)(n / 3)^{2} .
$$

Hence there exists $x \in\{u, v\}$ such that $x y z \in M$ for at least $(\varepsilon / 8)(n / 3)^{2}$ pairs $(y, z) \in Y \times Z$. In other words, $d_{M}(x)>(\varepsilon / 8)(n / 3)^{2} \geq \varepsilon^{\prime}(n / 3)^{2}$. This contradicts the Claim.

We may therefore suppose that the number of Type 2 copies of $F_{5}$ is at most $(2-2 \varepsilon / 3)(n / 3)^{2}$. Assume by symmetry that there are at most $(1-\varepsilon / 3)(n / 3)^{2}$ Type 2 copies of the form $u w z, e, v y z$. Arguing as above, the number of pairs $(y, z) \in Y \times Z$ for which either $u w z \in M$ or $v y z \in M$ is at least $(\varepsilon / 4)(n / 3)^{2}$. If at least half of the time we have $v y z \in M$, then we obtain $d_{M}(v)>(\varepsilon / 8)(n / 3)^{2} \geq \varepsilon^{\prime}(n / 3)^{2}$ and contradict the Claim. We therefore conclude that for at least $(\varepsilon / 8)(n / 3)^{2}$ pairs $(y, z) \in Y \times Z$, we have $u w z \in M$. Consequently, the number of $z \in Z$ for which $u w z \in M$ is at least $(\varepsilon / 10)(n / 3)$. We write this as $d_{M}(u w) \geq(\varepsilon / 10)(n / 3)$.

We have argued that for every edge $e=u v w \in B$ with $u, v$ in the same part and $w$ in a different part, either $d_{M}(u w) \geq \varepsilon n / 30$ or $d_{M}(v w) \geq \varepsilon n / 30$. Form a bipartite graph with parts $B$ and $M$. Let $e \in B$ be adjacent to $f \in M$ if $|e \cap f|=2$. We have shown above that each $e \in B$ has degree at least $\varepsilon n / 30$. Since $|B|>|M|$, we conclude that there exists $f \in M$ which is adjacent to at least $\varepsilon n / 30$ different $e \in B$. Each of these $e \in B$ has two points in common point with $f$, so there is a pair of vertices $u, v$ in different parts of $\mathcal{H}$ that lie is at least $\varepsilon n / 90$ different $e \in B$. Assume wlog that $u \in X, v \in Y$, and also that there are $x_{i} \in X$ for $1 \leq i \leq \varepsilon n / 180$ such that $u v x_{i} \in B$ for each $i$. For each $x_{i}$, consider $(y, z) \in(Y-\{v\}) \times Z$ and triples $x_{i} v z, x_{i} v u, u y z$. The number of such choices for $(i, y, z)$ is at least $(\varepsilon n / 200)(n / 3)^{2}$. If for at least half of these choices these three triples are edges of $\mathcal{H}$, then we obtain $\# F_{5} \geq(\varepsilon n / 400)(n / 3)^{2}>\delta n^{3}$ and we are done. So for at least half of these choices of $(i, y, z)$ we have either $x_{i} v z \in M$ or $u y z \in M$. Each such triple of $M$ is counted at most $n$ times so we obtain at least $(\varepsilon / 400)(n / 3)^{2}$ triples from $M$ incident to some vertex 
of $e$. At least one third of these triples are incident to the same vertex of $e$, so we obtain $x \in e$ with $d_{M}(x) \geq(\varepsilon / 1200)(n / 3)^{2} \geq \varepsilon^{\prime}(n / 3)^{2}$. The contradicts the Claim and completes the proof.

\subsection{Counting $B_{5}$ 's}

Theorem 6 follows from the following result. Recall that $c\left(n, B_{5}\right)=(2+o(1))(n / 3)^{2}$.

Theorem 18. For every $\varepsilon>0$ there exists $\delta>0$ and $n_{0}$ such that the following holds for $n>n_{0}$. Every $n$-vertex 3-graph with $b^{3}(n)+1$ edges contains either

- an edge that lies in at least $(2-\varepsilon)(n / 3)^{2}$ copies of $B_{5}$, or

- at least $\delta n^{4}$ copies of $B_{5}$.

Proof of Theorem 6. Remove $q-1$ edges from $\mathcal{H}$ and apply Theorem 18. If we find $\delta n^{4}$ copies of $B_{5}$, then since $q<\delta n^{2}$, the number of copies is much larger than $(1-\varepsilon) c\left(n, B_{5}\right)$ and we are done. Consequently, we find an edge $e_{1}$ in at least $(2-\varepsilon)(n / 3)^{2}>(1-\varepsilon) c\left(n, B_{5}\right)$ copies of $B_{5}$. Now remove $q-2$ edges from $\mathcal{H}-e_{1}$ and repeat this argument to obtain $e_{2}$. In this way we obtain edges $e_{1}, \ldots, e_{q}$ as required.

Sharpness follows by adding a partial Steiner triple system to $B^{3}(n)$ where each added edge is entirely within $X$. In other words, we are adding a collection of triples within $X$ such that every two have at most one point in common. It is easy to see that each added edge lies in $c\left(n, B_{5}\right)-O(1)$ copies of $B_{5}$ and moreover, since these edges have at most one common point, these copies are counted exactly once.

We will need the following stability theorem for $B_{5}$ proved by Füredi-Pikhurko-Simonovits $[9]$.

Theorem 19. ( $B_{5}$ stability [9]) Let $\mathcal{H}$ be a 3-graph with $n$ vertices and $b^{3}(n)-o\left(n^{3}\right)$ edges that contains no copy of $B_{5}$. Then there is a partition of the vertex set of $\mathcal{H}$ into $X \cup Y$ so that the number of edges that are not of the form $X X Y$ is o $\left(n^{3}\right)$. In other words, $\mathcal{H}$ can be obtained from $B^{3}(n)$ by adding and deleting a set of $o\left(n^{3}\right)$ edges.

Proof of Theorem 18. Given $\varepsilon$ let $0<\delta \ll \varepsilon$. Write $o_{\delta}(1)$ for any function that approaches zero as $\delta$ approaches zero and moreover, $o_{\delta}(1) \ll \varepsilon$. Let $n$ be sufficiently large 
and let $\mathcal{H}$ be an $n$-vertex 3 -graph with $b^{3}(n)+1$ edges. Write $\# B_{5}$ for the number of copies of $B_{5}$ in $\mathcal{H}$.

We first argue that we may assume that $\mathcal{H}$ has minimum degree at least $d=(4 / 9)\left(1-\delta_{1}\right)\left(\begin{array}{l}n \\ 2\end{array}\right)$, where $\delta_{1}=\delta^{1 / 4}$. Indeed, if this is not the case, then remove a vertex of degree less than $d$ to form the 3 -graph $\mathcal{H}_{1}$ with $n-1$ vertices. Continue removing a vertex of degree less than $d$ if such a vertex exists. If we could continue this process for $\delta_{2} n$ steps, where $\delta_{2}=\delta^{1 / 2}$, then the resulting 3 -graph $\mathcal{H}^{\prime}$ has $\left(1-\delta_{2}\right) n$ vertices and number of edges at least

$$
\frac{4}{9}\left(1-\delta-3 \delta_{2}\left(1-\delta_{1}\right)\left(\begin{array}{l}
n \\
3
\end{array}\right)>\frac{4}{9}(1+\delta)\left(\begin{array}{c}
\left(1-\delta_{2}\right) n \\
3
\end{array}\right) .\right.
$$

By the result of Füredi-Pikhurko-Simonovits [9] and Erdős-Simonovits supersaturation we conclude that $\mathcal{H}$ has at least $\delta^{\prime} n^{5}$ copies of $B^{5}$ (for some fixed $\delta^{\prime}>0$ ) and we are done. So we may assume that this process of removing vertices of degree less than $d$ terminates in at most $\delta_{2} n$ steps, and when it terminates we are left with a 3 -graph $\mathcal{H}^{\prime}$ on $n^{\prime}>\left(1-\delta_{2}\right) n$ vertices and minimum degree at least $d$.

Now suppose that we could prove that there is an edge of $\mathcal{H}^{\prime}$ that lies in at least $(2-$ $\varepsilon / 2)\left(n^{\prime} / 3\right)^{2}$ copies of $B_{5}$. Since $\delta \ll \varepsilon$, this is greater than $(2-\varepsilon)(n / 3)^{2}$ and we are done. If on the other hand $\mathcal{H}^{\prime}$ contains at least $2 \delta n^{\prime 4}$ copies of $B_{5}$, then again this is at least $\delta n^{4}$ and we are done. So if we could prove the result for $\mathcal{H}^{\prime}$ with $2 \delta, \varepsilon / 2$, then we could prove the result for $\mathcal{H}$ (with $\delta, \varepsilon$ ). Consequently, we may assume that $\mathcal{H}$ has minimum degree at least $\left(4 / 9-o_{\delta}(1)\right)\left(\begin{array}{l}n \\ 2\end{array}\right)$.

If $\# B_{5} \geq \delta n^{4}$, then we are done so assume that $\# B_{5}<\delta n^{4}$. Then by the Removal lemma, there is a set of at most $o_{\delta}\left(n^{3}\right)$ edges of $\mathcal{H}$ whose removal results in a 3 -graph $\mathcal{H}^{\prime}$ with no copies of $B_{5}$. Since $\left|\mathcal{H}^{\prime}\right|>b^{3}(n)-o_{\delta}\left(n^{3}\right)$, by Theorem 19 , we conclude that there is a partition $X \cup Y$ of the vertex set of $\mathcal{H}^{\prime}$ (and also of $\mathcal{H}$ ) such that the number of edges with 0,1 , or 3 points in $X$ is $o_{\delta}\left(n^{3}\right)$. Now pick a partition $X \cup Y$ of $\mathcal{H}$ that maximizes $e(X, X, Y)$ the number of edges with exactly two points in $X$. We know that $e(X, X, Y) \geq b^{3}(n)-o_{\delta}\left(n^{3}\right)$, and an easy calculation also shows that $|X|=2 n / 3+o_{\delta}(n)$ and $|Y|=n / 3+o_{\delta}(n)$.

Let $B$ be the set of edges of $\mathcal{H}$ that do not have exactly two points in $X$. Let $M$ be the set of triples with exactly two points in $X$ that are not edges of $\mathcal{H}$ and let $G=\mathcal{H}-B$ be the set of edges of $\mathcal{H}$ with exactly two points in $X$. Then $\mathcal{H}-B \cup M$ has a $(2,1)$-partition $X \cup Y$, so it has at most $b^{3}(n)$ edges. We conclude that

$$
|M|<|B|=o_{\delta}\left(n^{3}\right)
$$


In particular, $B \neq \emptyset$. Partition $B=B_{X X X} \cup B_{X Y Y} \cup B_{Y Y Y}$, where $B_{X^{i} Y^{3-i}}$ is the set of edges in $B$ with $i$ points in $X$ and $3-i$ points in $Y$.

Claim 1. For every vertex $v$ of $\mathcal{H}$ we have $d_{B_{X X X}}(v)<\varepsilon_{1} n^{2}$, where $\varepsilon_{1}=\varepsilon^{2} / 10^{6}$.

Proof of Claim 1. Suppose for contradiction that $d_{B_{X X X}}(v)>\varepsilon_{1} n^{2}$ for some vertex $v$. Let $B(v)$ be the set of edges in $B_{X X X}$ that contain $v$, so $|B(v)|=d_{B_{X X X}}(v)$. First observe that $d_{G}(v) \geq d_{B_{X X X}}(v)$, for otherwise we can move $v$ to $Y$ and contradict the choice of the partition $X, Y$. Now for each $e=v a b \in B(v)$ and $f=v x y \in \mathcal{H}$ with $\{a, b, x\} \in\left(\begin{array}{c}X-\{v\} \\ 3\end{array}\right), y \in$ $Y$, consider the two triples $a x y, b x y$. We see that $e, f, a x y, b x y$ forms a (potential) copy of $B_{5}$. For each $e$, the number of $f$ is at least $d_{G}(v)-n \geq|B(v)|-n>|B(v)| / 2$, since $f$ must omit $a, b$ and there are at most $|Y|$ pairs containing either of them. Hence the number of choices for $(e, f)$ is at least $|B(v)|^{2} / 2$. If for at least half of these choices of $(e, f)$, we obtain a copy of $B_{5}$ in $\mathcal{H}$, then $\# B_{5}>|B(v)|^{2} / 4>\delta n^{4}$, a contradiction. So for at least half of the choices of $(e, f)$ above, one of the triples $a x y, b x y$ is in $M$. A given triple in $M$ is counted at most $|X|<n$ times, so we obtain the contradiction $|B(v)|^{2} /(4 n)<|M|=o_{\delta}\left(n^{3}\right)$. This finishes the proof of the Claim.

Case 1. $\left|B_{X X X}\right| \geq|B| / 3$.

For each $e=u v w \in B_{X X X}$, and $(x, y) \in(X-e) \times Y$, there is a potential copy of $B_{5}$ consisting of vertices $u, v, w, x, y$ and edges $u x y, v x y, w x y, e$. This gives a total of $(|X|-3)|Y|>(2-$ $\left.o_{\delta}(1)\right)(n / 3)^{2}$ potential copies of $B_{5}$. At least $(2 \varepsilon / 3)(n / 3)^{2}$ of these potential copies of $B_{5}$ have a triple from $M$, for otherwise $e$ would lie in at least $\left(2-o_{\delta}(1)-2 \varepsilon / 3\right)(n / 3)^{2}>(2-\varepsilon)(n / 3)^{2}$ copies of $B_{5}$ and we are done. The triple from $M$ referenced above cannot be $e$ (since $e \in \mathcal{H}$ ), and therefore lies in exactly one copy of $B_{5}$ that was counted above. At least a third of these triples from $M$ are incident with the same vertex of $e$, hence there exists $z \in e$ such that $d_{M}(z)>(2 \varepsilon / 9)(n / 3)^{2}$.

Let $V=X \cup Y$ and let

$$
A=\left\{v \in V: d_{M}(v)>(2 \varepsilon / 9)(n / 3)^{2}\right\} .
$$

We have argued above that every $e \in B_{X X X}$ has a vertex in $A$. Consequently,

$$
9 \sum_{v \in A} d_{B_{X X X}}(v) \geq 9\left|B_{X X X}\right| \geq 3|B|>3|M| \geq \sum_{v \in A} d_{M}(v)>|A|(2 \varepsilon / 9)(n / 3)^{2},
$$

and there exists a vertex $v \in X \cap A$ such that $d_{B_{X X X}}(v)>(\varepsilon / 50)(n / 3)^{2}>\varepsilon_{1} n^{2}$. This contradicts Claim 1 and concludes the proof in this case. 
Case 2. $\left|B_{Y Y Y}\right| \geq|B| / 3$.

For each $e=u v w \in B_{Y Y Y}$ and $x, x^{\prime} \in X$, there is a potential copy of $B_{5}$ consisting of vertices $u, v, w, x, x^{\prime}$ and edges $x x^{\prime} u, x x^{\prime} v, x x^{\prime} w, e$. This gives a total of $\left(\begin{array}{c}|X| \\ 2\end{array}\right)>\left(2-o_{\delta}(1)\right)(n / 3)^{2}$ potential copies of $B_{5}$. At least $(2 \varepsilon / 3)(n / 3)^{2}$ of these potential copies of $B_{5}$ have a triple from $M$, for otherwise $e$ would lie in at least $\left(2-o_{\delta}(1)-2 \varepsilon / 3\right)(n / 3)^{2}>(2-\varepsilon)(n / 3)^{2}$ copies of $B_{5}$ and we are done. The triple from $M$ referenced above cannot be $e$ (since $e \in \mathcal{H}$ ), and therefore lies in exactly one copy of $B_{5}$. At least a third of these triples from $M$ are incident with the same vertex of $e$, hence there exists $z \in e$ such that $d_{M}(z)>(2 \varepsilon / 9)(n / 3)^{2}$. As in Case 1 , let $V=X \cup Y$ and $A=\left\{v \in V: d_{M}(v)>(2 \varepsilon / 9)(n / 3)^{2}\right\}$. We have argued above that every $e \in B_{Y Y Y}$ has a vertex in $A$. Consequently,

$$
9 \sum_{v \in A} d_{B_{Y Y Y}}(v) \geq 9\left|B_{Y Y Y}\right| \geq 3|B|>3|M| \geq \sum_{v \in A} d_{M}(v)>|A|(2 \varepsilon / 9)(n / 3)^{2},
$$

and there exists a vertex $v \in Y \cap A$ such that $d_{B_{Y Y Y}}(v)>(\varepsilon / 50)(n / 3)^{2}$. Let $B(v)$ be the set of edges in $B_{Y Y Y}$ that contain $v$, so $|B(v)|=d_{B_{Y Y Y}}(v)$.

Next we observe that $d_{B_{X Y Y}}(v) \leq d_{G}(v)$ otherwise we can move $v$ to $X$ and contradict the choice of the partition $X, Y$. We also recall that $\mathcal{H}$ has minimum degree at least $(4 / 9-$ $\left.o_{\delta}(1)\right)\left(\begin{array}{l}n \\ 2\end{array}\right)$, so

$$
d_{B_{Y Y Y}}(v)+d_{G}(v)+d_{B_{X Y Y}}(v) \geq\left(4 / 9-o_{\delta}(1)\right)\left(\begin{array}{l}
n \\
2
\end{array}\right) .
$$

Since $d_{B_{Y Y Y}}(v) \leq\left(\begin{array}{c}|Y| \\ 2\end{array}\right)<\left(1 / 9+o_{\delta}(1)\right)\left(\begin{array}{c}n \\ 2\end{array}\right)$, we conclude that

$$
d_{G}(v)>\frac{1}{2}\left(\frac{4}{9}-\frac{1}{9}-o_{\delta}(1)\right)\left(\begin{array}{l}
n \\
2
\end{array}\right)=\left(\frac{1}{6}-o_{\delta}(1)\right)\left(\begin{array}{l}
n \\
2
\end{array}\right) .
$$

Now for each $e=v y y^{\prime} \in B(v)$ and $f=v x x^{\prime} \in G\left(x, x^{\prime} \in X\right)$, consider the two triples $x x^{\prime} y, x x^{\prime} y^{\prime}$. We see that $e, f, x x^{\prime} y, x x^{\prime} y^{\prime}$ forms a potential copy of $B_{5}$. The number of choices of $(e, f)$ above is at least $|B(v)| d_{G}(v)=d_{B_{Y Y Y}}(v) d_{G}(v)$. If for at least half of these choices of $(e, f)$, we obtain a copy of $B_{5}$ in $\mathcal{H}$, then $\# B_{5}>d_{B_{Y Y Y}}(v) d_{G}(v) / 2>\delta n^{4}$, a contradiction. So for at least half of the choices of $(e, f)$ above, one of the triples $x x^{\prime} y, x x^{\prime} y^{\prime}$ is in $M$. A given triple in $M$ is counted at most $|Y|<n$ times, so we obtain the contradiction $d_{B_{Y Y Y}}(v) d_{G}(v) /(2 n)<|M|=o_{\delta}\left(n^{3}\right)$. This concludes the proof in this case.

Case 3. $\left|B_{X Y Y}\right| \geq|B| / 3$.

Let

$$
B_{1}=\left\{e \in B_{X Y Y}: \text { there exists } v \in e \cap Y \text { with } d_{M}(v)>\varepsilon(n / 3)^{2}\right\}
$$


Subcase 3.1. $\left|B_{1}\right| \geq\left|B_{X Y Y}\right| / 2$. Let

$$
A=\left\{v \in Y: d_{M}(v)>(\varepsilon / 2)(n / 3)^{2}\right\}
$$

By definition, every $e \in B_{1}$ has a vertex in $A$. Therefore

$$
18 \sum_{v \in A} d_{B_{1}}(v) \geq 18\left|B_{1}\right| \geq 9\left|B_{X Y Y}\right| \geq 3|B|>3|M| \geq \sum_{v \in A} d_{M}(v)>|A|(\varepsilon / 2)(n / 3)^{2}
$$

and there exists a vertex $v \in Y$ such that

$$
d_{B_{X Y Y}}(v) \geq d_{B_{1}}(v)>(\varepsilon / 36)(n / 3)^{2} .
$$

Recall that $G$ is the set of edges of $\mathcal{H}$ with exactly two points in $X$. Next observe that $d_{G}(v) \geq$ $d_{B_{X Y Y}}(v)$ for otherwise we can move $v$ to $X$ which increases $e(X, X, Y)$ and contradicts the choice of $X, Y$. It follows that $d_{G}(v)>(\varepsilon / 36)(n / 3)^{2}$.

Now for each $e=u v w \in B_{X Y Y}$ and $f=x x^{\prime} v \in G$ with $\left\{u, x, x^{\prime}\right\} \in\left(\begin{array}{c}X \\ 3\end{array}\right)$, and $w \in Y$, consider the two triples $u w x, u w x^{\prime}$. We see that $e, u w x, u w x^{\prime}, x x^{\prime} v$ forms a potential copy of $B_{5}$. The number of choices of $(e, f)$ above is at least $d_{B_{X Y Y}}(v) \times\left(d_{G}(v)-|X|\right)>d_{B_{X Y Y}}(v) d_{G}(v) / 2$. If for at least half of these choices of $(e, f)$, we obtain a copy of $B_{5}$ in $\mathcal{H}$, then

$$
\# B_{5}>\frac{d_{B_{X Y Y}}(v) d_{G}(v)}{4}>\frac{\varepsilon^{2}}{10^{5}}\left(\frac{n}{3}\right)^{4}>\delta n^{4}
$$

a contradiction. So for at least half of the choices of $(e, f)$ above, one of the triples $u w x, u w x^{\prime}$ is in $M$. A given triple in $M$ is counted at most $|X|<n$ times, so we obtain the contradiction

$$
\frac{\varepsilon^{2}}{10^{5}}\left(\frac{n^{3}}{3^{4}}\right)<\frac{d_{B_{X Y Y}}(v) d_{G}(v)}{4 n}<|M|=o_{\delta}\left(n^{3}\right) .
$$

This concludes the proof in this subcase.

Subcase 3.2. $\left|B_{1}\right|<\left|B_{X Y Y}\right| / 2$. So in this subcase we have $\left|B_{2}\right| \geq\left|B_{X Y Y}\right| / 2$, where

$$
B_{2}=\left\{e \in B_{X Y Y}: \text { for every } v \in e \cap Y \text { we have } d_{M}(v) \leq(\varepsilon / 2)(n / 3)^{2}\right\} \text {. }
$$

Fix $e=u v w \in B_{2}$ with $u \in X$ and $v, w \in Y$.

Claim 2. There exist sets $X_{v}, X_{w} \subset X$ such that

- $x u v \in M$ for every $x \in X_{v}$ and $x u w \in M$ for every $x \in X_{w}$ and

- $\left|X_{v}\right|>(\varepsilon / 20) n$ and $\left|X_{w}\right|>(\varepsilon / 20) n$ 
Proof of Claim 2. Let $X_{v}=\{x \in X: x u v \in M\}$. We will show that $\left|X_{v}\right| \geq(\varepsilon / 20) n$. The same argument will apply to $X_{w}$.

Suppose for contradiction that $\left|X_{v}\right|<(\varepsilon / 20) n$. Pick $x, x^{\prime} \in X-X_{v}$ and consider $u, v, w, x, x^{\prime}$. The triples $u v x, u v x^{\prime}, e, x x^{\prime} w$ form a potential copy of $B_{5}$. Since $x, x^{\prime} \in X-X_{v}$, we have $u v x \in \mathcal{H}$ and $u v x^{\prime} \in \mathcal{H}$. So if these four edges do not form a copy of $B_{5}$ in $\mathcal{H}$ then $x x^{\prime} w \in M$. Since $e \in B_{2}$, the number of pairs $\left\{x, x^{\prime}\right\} \in\left(\begin{array}{c}X \\ 2\end{array}\right)$ such that $x x^{\prime} w \in M$ is at most $(\varepsilon / 2)(n / 3)^{2}$. Consequently, the number of pairs $x, x^{\prime} \in X-X_{v}$ with $x x^{\prime} w \in \mathcal{H}$ is at least

$$
\begin{aligned}
\left(\begin{array}{c}
\left|X-X_{v}\right|-1 \\
2
\end{array}\right)-\frac{\varepsilon}{2}\left(\frac{n}{3}\right)^{2} & >\left(\begin{array}{c}
\left(1-o_{\delta}(1)-\frac{3 \varepsilon}{40}\right) \frac{2 n}{3} \\
2
\end{array}\right)-\frac{\varepsilon}{2}\left(\frac{n}{3}\right)^{2} \\
& >\left(2\left(1-\frac{\varepsilon}{10}\right)^{2}-\frac{\varepsilon}{2}\right)\left(\frac{n}{3}\right)^{2} \\
& =\left(2-\frac{9 \varepsilon}{10}+\frac{\varepsilon^{2}}{50}\right)\left(\frac{n}{3}\right)^{2} \\
& >(2-\varepsilon)\left(\frac{n}{3}\right)^{2} .
\end{aligned}
$$

This gives us the required number of copies of $B_{5}$ containing the edge $e$ and concludes the proof of the Claim.

For each edge $e=u v w \in B_{2}$ with $u \in X, v, w \in Y$, Claim 2 shows that are at least $(\varepsilon / 20) n$ triples of the form $x u v \in M$. Form the bipartite graph with parts $B_{2}$ and $M$, where $u v w \in B_{2}$ is adjacent to all such $x u v \in M$. Then since every vertex of $B_{2}$ has degree at least $(\varepsilon / 20) n$, and $\left|B_{2}\right| \geq\left|B_{X Y Y}\right| / 2 \geq|B| / 6>|M| / 6$, we conclude that there exists $x u v \in M$ (with $v \in Y$ ) which is adjacent to at least $(\varepsilon / 120) n$ edges in $B_{2}$. Each of these edges of $B_{2}$ contains $v$, and either $x$ or $u$, so we may assume by symmetry that at least half of them contain $u$. So we have $u v w_{i} \in B_{2}$, where $u \in X$ and $v, w_{i} \in Y$ for $i=1, \ldots,(\varepsilon / 240) n$. For each $w_{i}$, consider the set $X_{w_{i}}$ defined in Claim 2. We know that $x^{\prime} u w_{i} \in M$ for each $w_{i}$ and $x^{\prime} \in X_{w_{i}}$. Since these triples are distinct for distinct $w_{i}$ or distinct $x^{\prime}$, we conclude that $d_{M}(u) \geq(\varepsilon / 240) n(\varepsilon / 20) n=\left(\varepsilon^{2} / 4800\right) n^{2}$. Recalling the minimum degree condition on $\mathcal{H}$, we have

$$
\left(4 / 9-o_{\delta}(1)\right)\left(\begin{array}{l}
n \\
2
\end{array}\right) \leq d_{\mathcal{H}}(u)=d_{G}(u)+d_{B}(u) \leq\left(4 / 9-o_{\delta}(1)\right)\left(\begin{array}{l}
n \\
2
\end{array}\right)-d_{M}(u)+d_{B}(u) .
$$

We conclude that $d_{B}(u) \geq\left(\varepsilon^{2} / 5000\right) n^{2}$. By Claim 1 we know that $d_{B_{X X X}}(u)<\varepsilon_{1} n^{2}$ where $\varepsilon_{1}=\varepsilon^{2} / 10^{5}$. As $d_{B}(u)=d_{B_{X X X}}(u)+d_{B_{X Y Y}}(u)$, we obtain

$$
d:=d_{B_{X Y Y}}(u)=d_{B}(u)-d_{B_{X X X}}(u)>\left(\varepsilon^{2} / 5000\right) n^{2}-\varepsilon_{1} n^{2} \geq 2 \varepsilon_{1} n^{2} .
$$


Say that $u y y^{\prime} \in B_{X Y Y}$ is bad if

$$
\mid\left\{x \in X: x u y \in M \text { or } x u y^{\prime} \in M\right\} \mid>\left(1-\varepsilon_{1}\right)(2 n / 3) .
$$

Let

$$
S=\left\{\left\{y, y^{\prime}\right\} \in\left(\begin{array}{c}
|Y| \\
2
\end{array}\right): u y y^{\prime} \text { is bad }\right\} .
$$

Now suppose that $|S| \geq(0.9) d$. For each $e=u y y^{\prime} \in B_{X Y Y}$ with $\left\{y, y^{\prime}\right\} \in S$ there is a set $X_{e} \subset X$ with $\left|X_{e}\right| \geq\left(1-\varepsilon_{1}\right)(2 n / 3)$ such that $x u y \in M$ or $x u y^{\prime} \in M$ for all $x \in X_{e}$. Each of these triples in $M$ is counted at most $|Y|$ times so we obtain

$$
d_{M}(u) \geq \frac{|S|\left(1-\varepsilon_{1}\right)(2 n / 3)}{\left(1+o_{\delta}(1)\right) n / 3}=2\left(1-2 \varepsilon_{1}\right)|S| \geq(1.8)\left(1-2 \varepsilon_{1}\right) d>(1.7) d .
$$

Again recalling the minimum degree condition on $\mathcal{H}$, we have

$$
\begin{aligned}
\left(4 / 9-o_{\delta}(1)\right)\left(\begin{array}{l}
n \\
2
\end{array}\right) \leq d_{\mathcal{H}}(u) & =d_{B_{X X X}}(u)+d_{G}(u)+d_{B_{X Y Y}}(u) \\
& =d_{B_{X X X}}(u)+\left(\left(4 / 9+o_{\delta}(1)\right)\left(\begin{array}{l}
n \\
2
\end{array}\right)-d_{M}(u)\right)+d
\end{aligned}
$$

Using (6) and $d>\varepsilon_{1} n^{2}$ we obtain $d_{B_{X X X}}(u)>(0.7) d-o_{\delta}\left(n^{2}\right)>\varepsilon_{1} n^{2}$. This contradicts Claim 1 and concludes the proof if $|S| \geq(0.9) d$.

Next suppose that $|S|<(0.9) d$. So for at least $(0.1) d$ edges $e=u y y^{\prime} \in B_{X Y Y}$ we have a set $X_{e} \subset X$ such that

$$
\left|X_{e}\right| \geq\left(\varepsilon_{1}-o_{\delta}(1)\right)(2 n / 3)>\left(\varepsilon_{1} / 3\right) n
$$

and $u y x \in \mathcal{H}$ for all $x \in X_{e}$ (also $u y^{\prime} x \in \mathcal{H}$ but we wont use this).

Let $x, x^{\prime} \in X_{e}$ and consider the triple $x x^{\prime} y^{\prime}$. We see that $e, u y x, u y x^{\prime}, x x^{\prime} y^{\prime}$ forms a potential copy of $B_{5}$. The number of choices for $\left(e,\left\{x, x^{\prime}\right\}\right)$ above is at least

$$
(0.1) d \times\left(\begin{array}{c}
\left(\varepsilon_{1} / 3\right) n \\
2
\end{array}\right)>\left(\varepsilon_{1} / 5\right) n^{2} \times\left(\varepsilon_{1}^{2} / 20\right) n^{2}=\left(\varepsilon_{1}^{3} / 100\right) n^{4} .
$$

If for at least half of these choices of $\left(e,\left\{x, x^{\prime}\right\}\right)$, we have $x x^{\prime} y^{\prime} \in \mathcal{H}$, then

$$
\# B_{5}>\left(\varepsilon_{1}^{3} / 200\right) n^{4}>\delta n^{4}
$$

a contradiction. So for at least half of the choices of $\left(e,\left\{x, x^{\prime}\right\}\right)$ above, $x x^{\prime} y^{\prime} \in M$. A given triple $x x^{\prime} y^{\prime} \in M$ is counted at most $|Y|<n / 2$ times, so we obtain the contradiction

$$
\frac{\varepsilon_{1}^{3}}{200} n^{3} \leq \frac{(0.1) d \times\left(\begin{array}{c}
\left|X_{e}\right| \\
2
\end{array}\right)}{n}<|M|=o_{\delta}\left(n^{3}\right) .
$$

This completes the proof of the subcase and the Theorem. 


\subsection{Counting Expansions of Cliques}

In this section we will prove Theorems 7 and 8 .

\subsubsection{Asymptotic Counting}

Theorem 7 follows from the following result. Recall that $l_{r+1}=\left(\begin{array}{c}r+2 \\ 2\end{array}\right)$ is the number of vertices of $L_{r+1}$ and $c\left(n, L_{r+1}\right)=\Theta\left(n^{l_{r+1}-3}\right)$.

Theorem 20. For every $\varepsilon>0$ there exists $\delta>0$ and $n_{0}$ such that the following holds for $n>n_{0}$. Every $n$ vertex 3-graph with $t_{r}^{3}(n)+1$ edges contains either

- at least $\delta n^{l_{r+1}-1}$ copies of $L_{r+1}$, or

- an edge that lies in at least $c\left(n, L_{r+1}\right)$ copies of $L_{r+1}$, or

- two edges that each lie in at least $(1-\varepsilon) c\left(n, L_{r+1}\right)$ copies of $L_{r+1}$ with none of these copies containing both edges.

Proof of Theorem 7. Remove $q-1$ edges from $\mathcal{H}$ and apply Theorem 20. If we find $\delta n^{l_{r+1}-1}$ copies of $L_{r+1}$, then since $q<\delta n^{2}$, the number of copies is much larger than $q(1-\varepsilon) c\left(n, L_{r+1}\right)$ and we are done. Consequently, we find an edge $e_{1}$ in at least $(1-\varepsilon) c\left(n, L_{r+1}\right)$ copies of $L_{r+1}$. Now remove $q-2$ edges from $\mathcal{H}-e_{1}$ and repeat this argument to obtain $e_{2}$. In this way we obtain edges $e_{1}, \ldots, e_{q}$ as required.

Sharpness follows by the following construction: Take $T_{r}^{3}(n)$ with parts $V_{1}, \ldots, V_{r}$, pick any point $y \in V_{2}$, and add $q$ edges of the form $x x^{\prime} y$ with $x, x^{\prime} \in V_{1}$. Each added edge lies in at most $(1+\varepsilon) c\left(n, L_{r+1}\right)$ copies of $L_{r+1}$, and no two added edges lie in a common copy of $L_{r+1}$, since $L_{r+1}$ has the property that for every two edges $e, e^{\prime}$ containing a common vertex $v$, there is another edge $f$ containing a point from each of $e-\{v\}$ and $e^{\prime}-\{v\}$ and $v \notin f$. Taking two edges containing $y$, we see that there is no edge that can play the role of $f$ above.

We will need the following stability result proved by Pikhurko [24] (see also [18]).

Theorem 21. ( $L_{r+1}$ Stability [24]) Let $\mathcal{H}$ be a 3-graph with $n$ vertices and $t_{r}^{3}(n)-o\left(n^{3}\right)$ edges that contains no copy of $L_{r+1}$. Then there is a partition of the vertex set of $\mathcal{H}$ into $r$ 
parts so that the number of edges that intersect some part in at least two points is o $\left(n^{3}\right)$. In other words, $\mathcal{H}$ can be obtained from $T_{r}^{3}(n)$ by adding and deleting a set of o $\left(n^{3}\right)$ edges.

Proof of Theorem 20. Given $\varepsilon$ let $0<\delta \ll \varepsilon$. Write $o_{\delta}(1)$ for any function that approaches zero as $\delta$ approaches zero and moreover, $o_{\delta}(1) \ll \varepsilon$. Let $n$ be sufficiently large and let $\mathcal{H}$ be an $n$ vertex 3-graph with $t_{r}^{3}(n)+1$ edges. Write $\# L_{r+1}$ for the number of copies of $L_{r+1}$ in $\mathcal{H}$.

If $\# L_{r+1} \geq \delta n^{l_{r+1}-1}$, then we are done so assume that $\# L_{r+1}<\delta n^{l_{r+1}-1}$. Then by the Removal lemma, there is a set of at most $o_{\delta}\left(n^{3}\right)$ edges of $\mathcal{H}$ whose removal results in a 3 -graph $\mathcal{H}^{\prime}$ with no copies of $L_{r+1}$. Since $\left|\mathcal{H}^{\prime}\right|>t_{r}^{3}(n)-o_{\delta}\left(n^{3}\right)$, by Theorem 21 , we conclude that there is an $r$-partition $V_{1} \cup \cdots \cup V_{r}$ of $\mathcal{H}^{\prime}$ (and also of $\mathcal{H}$ ) such that the number of edges that intersect some part in at least two points is $o_{\delta}\left(n^{3}\right)$. Now pick a partition $V_{1} \cup \cdots \cup V_{r}$ of $\mathcal{H}$ that maximizes $h_{1}+2 h_{2}+3 h_{3}$, where $h_{i}$ is the number of edges of $\mathcal{H}$ that intersect precisely $i$ of the parts. The partition guaranteed by Theorem 21 satisfies $h_{1}+2 h_{2}=o_{\delta}\left(n^{3}\right)$, and hence for this particular partition $h_{1}+2 h_{2}+3 h_{3} \geq 3|\mathcal{H}|-2\left(h_{1}+h_{2}\right)>3 t_{r}^{3}(n)-o_{\delta}\left(n^{3}\right)$. Since $h_{1}+2 h_{2}+3 h_{3} \leq 3|\mathcal{H}|-\left(h_{1}+h_{2}\right)$ we conclude that for the partition that maximizes $h_{1}+2 h_{2}+3 h_{3}$ we have $h_{1}+2 h_{2}=o_{\delta}\left(n^{3}\right)$ and $h_{3} \geq t_{r}^{3}(n)-o_{\delta}\left(n^{3}\right)$. A standard calculation also shows that for this partition each $V_{i}$ has size $n / r \pm o_{\delta}(n)$.

Let $B=\mathcal{H}-\prod_{i=1}^{r} V_{i}$, let $G=\mathcal{H}-B$ and $M=\prod_{i=1}^{r} V_{i}-G$. Then $\mathcal{H}-B \cup M$ is $r$-partite so it has at most $t_{r}^{3}(n)$ edges. We conclude that

$$
|M|<|B|=o_{\delta}\left(n^{3}\right)
$$

in particular $|B| \geq 1$. We will now argue that we can improve this to $|B| \geq 2$. We may suppose that $n_{i}:=\left|V_{i}\right|$ satisfy $n_{1} \geq n_{2} \geq \ldots \geq n_{r}$. Pick $e_{1} \in B$. If $\mathcal{H}-e_{1} \cong T_{r}^{3}(n)$, then clearly $e_{1}$ lies in at least $c\left(n, L_{r+1}\right)$ copies of $L_{r+1}$ and we are done. So assume that $\mathcal{H}-e_{1} \neq T_{r}^{3}(n)$. Suppose that $B \cap\left(\mathcal{H}-e_{1}\right)=\emptyset$. Then either $n_{r} \geq n_{1}-1$ and

$$
t_{r}^{3}(n)=\left|\mathcal{H}-e_{1}\right| \leq\left(\sum_{S \in\left(\begin{array}{c}
{[r]} \\
3
\end{array}\right)} \prod_{i \in S} n_{i}\right)-1<t_{r}^{3}(n),
$$

or $n_{r}<n_{1}-1$ and

$$
t_{r}^{3}(n)=\left|\mathcal{H}-e_{1}\right| \leq \sum_{S \in\left(\begin{array}{c}
{[r]} \\
3
\end{array}\right)} \prod_{i \in S} n_{i}<t_{r}^{3}(n)
$$


In either case we have a contradiction, so we may assume that $B \cap\left(\mathcal{H}-e_{1}\right) \neq \emptyset$. In other words, there exists $e_{2} \neq e_{1}$ such that $e_{2} \in B$ and therefore $|B| \geq 2$. We will now show that every $e \in B$ lies in at least $(1-\varepsilon) c\left(n, L_{r+1}\right)$ copies of $L_{r+1}$ in $\mathcal{H}$ and each copy uses a unique edge from $B$.

Let $e=x y z \in B$. We may assume by symmetry that $x, y \in V_{1}$. Pick $\left(v_{2}, \ldots, v_{r}\right) \in$ $V_{2} \times \cdots \times V_{r}$ with $v_{i} \neq z$ for all $i$. For every pair of distinct vertices $\{a, b\}$ with $a \in\left\{v_{2}, \ldots, v_{r}\right\}$ and $b \in\left\{x, y, v_{2}, \ldots, v_{r}\right\}$ (there are $\left(\begin{array}{c}r-1 \\ 2\end{array}\right)+2(r-1)$ such $\left.\{a, b\}\right)$, let $v_{a b}$ be a vertex in a part different from $a, b$ that is distinct from all other vertices being considered. The number of choices for the $\left(\left(\begin{array}{c}r+1 \\ 2\end{array}\right)+r-2\right)$-tuple $\left(v_{2}, \ldots, v_{r},\left\{v_{a b}\right\}_{a, b}\right)$ is at least $\left(1-o_{\delta}(1)\right) c\left(n, L_{r+1}\right)$. Moreover, the $\left(\begin{array}{c}r+1 \\ 2\end{array}\right)$ edges $e$ and $\left\{a b v_{a b}\right\}_{a, b}$ form a potential copy of $L_{r+1}$ with $x, y, v_{2}, \ldots, v_{r}$ forming the original $K_{r+1}$ whose edges have been expanded. At least $(\varepsilon / 2) c\left(n, L_{r+1}\right)$ of these potential copies of $L_{r+1}$ have a triple from $M$, otherwise $e$ would lie in at least (1$\left.o_{\delta}(1)-\varepsilon / 2\right) c\left(n, L_{r+1}\right)>(1-\varepsilon) c\left(n, L_{r+1}\right)$ copies of $L_{r+1}$ and we are done. Suppose that at least $(\varepsilon / 4) c\left(n, L_{r+1}\right)$ of these potential copies of $L_{r+1}$ have the triple from $M$ omitting $e$. Since each such triple from $M$ is counted at most $n^{l_{r+1}-6}$ times, we obtain the contradiction $(\varepsilon / 4) c\left(n, L_{r+1}\right) / n^{l_{r+1}-6} \leq|M|<o_{\delta}\left(n^{3}\right)$. So at least $(\varepsilon / 4) c\left(n, L_{r+1}\right)$ of these potential copies of $L_{r+1}$ have a triple from $M$ containing $x$ or $y$. Each such triple from $M$ is counted at most $n^{l_{r+1}-5}$ times, so there are at least $(\varepsilon / 4) c\left(n, L_{r+1}\right) / n^{l_{r+1}-5}=\varepsilon^{\prime} n^{2}$ triples from $M$ containing $x$ or $y$ (for suitable $\varepsilon^{\prime}>0$ depending only on $r$ ). We may assume by symmetry that $d_{M}(x)>\left(\varepsilon^{\prime} / 2\right) n^{2}$.

We have shown above that for each $e \in B$, there is a vertex $x \in e$ that lies in the (unique) part that has at least two points from $e$, with $d_{M}(x)>\left(\varepsilon^{\prime} / 2\right) n^{2}$. Form a bipartite graph with parts $B$ and $M$, where each $e \in B$ is adjacent to those $f$ in $M$ for which $e \cap f=\{x\}$ and $x$ lies in the part that has at least two points of $e$. Then each vertex of $B$ has degree at least $\left(\varepsilon^{\prime} / 2\right) n^{2}$. Since $|B|>|M|$ we conclude that there exists $f \in M$ adjacent to at least $\left(\varepsilon^{\prime} / 2\right) n^{2}$ different $e \in B$ in the way specified above. At least $\left(\varepsilon^{\prime} / 6\right) n^{2}$ of these $e \in B$ contain the same point $x \in f$. Assume wlog that $x \in V_{1}$.

For each $i \in[r]$ and $\varepsilon_{1}=\varepsilon^{\prime} / 100$, define

$$
A_{i}=\left\{y \in V_{i}: d_{\mathcal{H}}(x y) \geq \varepsilon_{1} n\right\} .
$$

Claim. $\left|A_{i}\right|<\varepsilon_{1} n$ for some $i \in[r]$.

Proof of Claim. Suppose to the contrary that $\left|A_{i}\right| \geq \varepsilon_{1} n$ for each $i$. Then the number 
of choices $\left(v_{1}, \ldots, v_{r}\right) \in A_{1} \times \cdots \times A_{r}$ is at least $\left(\varepsilon_{1} n\right)^{r}$. For every pair of distinct vertices $\{a, b\} \subset\left\{v_{1}, \ldots, v_{r}\right\}$, let $w_{a b} \neq x$ be a vertex in a part different from $a, b$ (there are at least $(1-2 / r) n>n / 2 r$ choices for $\left.w_{a b}\right)$. For every vertex $c \subset\left\{v_{1}, \ldots, v_{r}\right\}$, let $w_{c}$ be a vertex such that $x c w_{c} \in \mathcal{H}$. By definition of $A_{i}$, we know that the number of such $w_{c}$ is at least $\varepsilon_{1} n$. Consequently, the number of choices for the $\left(l_{r+1}-1\right)$-tuple of distinct vertices $\left(v_{1}, \ldots, v_{r},\left\{w_{a b}\right\}_{a, b},\left\{w_{c}\right\}_{c}\right)$ is at least

$$
\left(\varepsilon_{1} n\right)^{r}(n / 2 r)^{\left(\begin{array}{l}
r \\
2
\end{array}\right)}\left(\varepsilon_{1} n\right)^{r}>\left(\varepsilon_{1} / r\right)^{r^{2}} n^{l_{r+1}-1}=\varepsilon_{2} n^{l_{r+1}-1} .
$$

Moreover, the $\left(\begin{array}{c}r+1 \\ 2\end{array}\right)$ triples $x c w_{c}, a b w_{a b}$ over all choices of $a, b, c$ form a potential copy of $L_{r+1}$ with $x, v_{1}, \ldots, v_{r}$ forming the original $K_{r+1}$ whose edges have been expanded. At least $\left(\varepsilon_{2} / 2\right) n^{l_{r+1}-1}$ of these potential copies of $L_{r+1}$ have a triple from $M$, otherwise $\# L_{r+1} \geq$ $\left(\varepsilon_{2} / 2\right) n^{l_{r+1}-1}>\delta n^{l_{r+1}-1}$ and we are done. Each such triple from $M$ omits $x$ and is therefore counted at most $n^{l_{r+1}-4}$ times (since $x$ is fixed and $L_{r+1}$ has $l_{r+1}$ vertices) so we obtain the contradiction $\left(\varepsilon_{2} / 2\right) n^{l_{r+1}-1} / n^{l_{r+1}-4} \leq|M|<o_{\delta}\left(n^{3}\right)$. This completes the proof of the Claim.

Let $B(x)$ be the set of edges of $B$ containing $x$ with at least two vertices in $V_{1}$. Then we had earlier shown that $|B(x)| \geq\left(\varepsilon^{\prime} / 6\right) n^{2}>10 \varepsilon_{1} n^{2}$.

Let $H(x)$ be the set of pairs $\{y, z\}$ such that $x y z \in B(x)$, so one of $y, z \in V_{1}$ and $|H(x)|=$ $|B(x)|$. Now $\left|A_{1}\right| \geq \varepsilon_{1} n$ for otherwise we obtain the contradiction

$$
|B(x)| \leq \sum_{v \in V_{1}} d_{H(x)}(v)=\sum_{v \in A_{1}} d_{H(x)}(v)+\sum_{v \in V_{1}-A_{1}} d_{H(x)}(v) \leq\left(\varepsilon_{1} n\right) n+(n / 2)\left(\varepsilon_{1} n\right)<2 \varepsilon_{1} n^{2} .
$$

The Claim implies that one of $\left|A_{2}\right|, \ldots,\left|A_{r}\right|$ is less than $\varepsilon_{1} n$. By symmetry, we may assume that $\left|A_{r}\right|<\varepsilon_{1} n$. The number of edges in $\mathcal{H}$ containing $x$ and some vertex of $V_{r}$ is at most $\left|A_{r}\right| n+\left|V_{r}\right| \varepsilon_{1} n<2 \varepsilon_{1} n^{2}$. Hence the number of edges in $B(x)$ that have no vertex in $V_{r}$ is at least $|B(x)|-2 \varepsilon_{1} n^{2}>8 \varepsilon_{1} n^{2}$.

Now let us contemplate moving $x$ from $V_{1}$ to $V_{r}$. The edges of $\mathcal{H}$ containing $x$ whose contribution to $\sum_{i} i h_{i}$ decreases (by at most one) must have a vertex in $V_{r}$, and their number is at most $2 \varepsilon_{1} n^{2}$. The edges in $B(x)$ that have no vertex in $V_{r}$ give an increased contribution to $\sum_{i} i h_{i}$ (each edge contributes an increase of exactly one), and their number is at least $8 \varepsilon_{1} n^{2}$. All other edges containing $x$ (i.e. those with $r-1$ vertices in $V_{2} \cup \cdots \cup V_{r-1}$ ) do not change their contribution to $\sum_{i} i h_{i}$. The net contribution to $\sum_{i} i h_{i}$ therefore increases by at least $6 \varepsilon_{1} n^{2}>0$, thus contradicting the choice of the partition and completing the proof. 


\subsubsection{Exact Counting}

In this subsection we will use Theorem 20 to prove Theorem 8 .

Proof of Theorem 8. Given $q \geq 1$, let $0<\varepsilon \leq 1 /(q+1)$. Then $2(1-\varepsilon) c\left(n, L_{r+1}\right) \geq$ $c\left(n, L_{r+1}\right)$. Let $\delta$ and $n_{0}$ be the outputs of Theorem 7 with input $\varepsilon$. Choose $n>n_{0}$ such that it also satisfies $\delta n^{l_{r+1}-1}>q \times c\left(n, L_{r+1}\right)$ (this is a triviality since $c\left(n, L_{r+1}\right)=O\left(n^{l_{r+1}-3}\right)$ ).

Suppose that $\mathcal{H}$ is an $n$ vertex 3 -graph with $t_{r}^{3}(n)+q$ edges. Write $\# L_{r+1}$ for the number of copies of $L_{r+1}$ in $\mathcal{H}$. Let us prove by induction on $q$ that $\# L_{r+1} \geq q \times c\left(n, L_{r+1}\right)$. If $q=1$, then Theorem 20 and the definitions of $\varepsilon, \delta, n$ imply that

$$
\# L_{r+1} \geq \min \left\{\delta n^{l_{r+1}-1}, c\left(n, L_{r+1}\right), 2(1-\varepsilon) c\left(n, L_{r+1}\right)\right\} \geq c\left(n, L_{r+1}\right) .
$$

Let us assume that $q>1$ and the result holds for $q-1$.

Let $e_{1}$ be an edge of $\mathcal{H}$ that lies in the maximum number of copies of $L_{r+1}$, say that it lies in $c_{1}(n)$ copies. If $c_{1}(n) \geq c\left(n, L_{r+1}\right)$, then let $\mathcal{H}_{1}=\mathcal{H}-e_{1}$. By induction, $\mathcal{H}_{1}$ has at least $(q-1) c\left(n, L_{r+1}\right)$ copies of $L_{r+1}$. These copies are distinct from those containing $e_{1}$ so we obtain

$$
\# L_{r+1} \geq c_{1}(n)+(q-1) c\left(n, L_{r+1}\right) \geq q c\left(n, L_{r+1}\right)
$$

and we are done.

We may therefore assume that $c_{1}(n)<c\left(n, L_{r+1}\right)$. Let $e_{2}$ be an edge of $\mathcal{H}_{1}$ that lies in the maximum number $c_{2}(n)$ of copies of $L_{r+1}$ in $\mathcal{H}_{1}$. Since $\mathcal{H}_{1} \subset \mathcal{H}$, clearly $c_{2}(n) \leq c_{1}(n)$. Let $\mathcal{H}_{2}=\mathcal{H}_{1}-e_{2}$ and continue this process to obtain $e_{1}, \ldots, e_{q-1}$. For each $i \leq q-1$, Theorem 20 implies that $\# L_{r+1} \geq \delta n^{l_{r+1}-1}>q c\left(n, L_{r+1}\right)$ or $c_{i}(n) \geq(1-\varepsilon) c\left(n, L_{r+1}\right)$. In the former case we are done, so we may assume that

$$
(1-\varepsilon) c\left(n, L_{r+1}\right) \leq c_{q-1}(n) \leq \cdots \leq c_{1}(n)<c\left(n, L_{r+1}\right) .
$$

Consider $\mathcal{H}_{q-1}=\mathcal{H}-e_{1}-e_{2} \ldots-e_{q-1}$. Then

$$
\left|\mathcal{H}_{q-1}\right|=|\mathcal{H}|-(q-1)=t_{r}^{3}(n)+q-(q-1)=t_{r}^{3}(n)+1 \text {. }
$$

Since $c_{q-1}(n)<c\left(n, L_{r+1}\right)$, Theorem 20 implies that $\mathcal{H}_{q-1}$ has at least $2(1-\varepsilon) c\left(n, L_{r+1}\right)$ copies of $L_{r+1}$. Altogether we have

$$
\# L_{r+1} \geq 2(1-\varepsilon) c\left(n, L_{r+1}\right)+\sum_{i=1}^{q-1} c_{i}(n) \geq(1-\varepsilon)(q+1) c\left(n, L_{r+1}\right) \geq q c\left(n, L_{r+1}\right)
$$

where the last equality follows from $\varepsilon \leq 1 /(q+1)$. This completes the proof. 


\subsection{Counting $P_{2}$ 's}

Recall that $c\left(n, P_{2}\right)=2(n / 4)^{3}+\Theta\left(n^{2}\right)$. Theorem 9 for $l=2$ follows from the following result.

Theorem 22. For every $\varepsilon>0$ there exists $\delta>0$ and $n_{0}$ such that the following holds for $n>n_{0}$. Every $n$ vertex 4-graph with $t^{4}(n)+1$ edges contains either

- an edge that lies in at least $(2-\varepsilon)(n / 4)^{3}$ copies of $P_{2}$, or

- at least $\delta n^{4}$ copies of $P_{2}$.

Proof of Theorem 9 for $l=2$. Remove $q-1$ edges from $\mathcal{H}$ and apply Theorem 22 . If we find $\delta n^{4}$ copies of $P_{2}$, then since $q<\delta n$, the number of copies is much larger than $(1-\varepsilon) q c\left(n, P_{2}\right)$ and we are done. Consequently, we find an edge $e_{1}$ in at least $(2-\varepsilon)(n / 4)^{3}>$ $(1-\varepsilon) c\left(n, P_{2}\right)$ copies of $P_{3}$. Now remove $q-2$ edges from $\mathcal{H}-e_{1}$ and repeat this argument to obtain $e_{2}$. In this way we obtain edges $e_{1}, \ldots, e_{q}$ as required.

The bound is sharp due to the following construction. Suppose that $T^{4}(n)$ has parts $W, X, Y, Z$. Fix a pair of points $a, b \in W$, and add $q$ edges of the form $a b c d$ where $c, d \in X$. It is easy to see that each added edge lies in $2(n / 4)^{3}+O\left(n^{2}\right)$ copies of $P_{2}$ and no copy of $P_{2}$ contains two of the new edges. Consequently, the copies of $P_{2}$ are counted exactly once.

We will need the following stability theorem for $P_{2}$ proved by Pikhurko [24]

Theorem 23. ( $P_{2}$ stability [24]) Let $\mathcal{H}$ be a 4-graph with $n$ vertices and $t^{4}(n)-o\left(n^{4}\right)$ edges that contains no copy of $P_{2}$. Then there is a partition of the vertex set of $\mathcal{H}$ into $W \cup X \cup Y \cup Z$ so that the number of edges that intersect a part in at least two points is $o\left(n^{4}\right)$. In other words, $\mathcal{H}$ can be obtained from $T^{4}(n)$ by adding and deleting a set of o $\left(n^{4}\right)$ edges.

Proof of Theorem 22. Given $\varepsilon$ let $0<\delta \ll \varepsilon$. Write $o_{\delta}(1)$ for any function that approaches zero as $\delta$ approaches zero and moreover, $o_{\delta}(1) \ll \varepsilon$. Let $n$ be sufficiently large and let $\mathcal{H}$ be an $n$ vertex 4 -graph with $t^{4}(n)+1$ edges. Write $\# P_{2}$ for the number of copies of $P_{2}$ in $\mathcal{H}$.

We first argue that we may assume that $\mathcal{H}$ has minimum degree at least $d=(3 / 32)\left(1-\delta_{1}\right)\left(\begin{array}{l}n \\ 3\end{array}\right)$, where $\delta_{1}=\delta^{1 / 4}$. Indeed, if this is not the case, then remove a vertex of degree less than $d$ to form the 4 -graph $\mathcal{H}_{1}$ with $n-1$ vertices. Continue removing a vertex of degree less than $d$ 
if such a vertex exists. If we could continue this process for $\delta_{2} n$ steps, where $\delta_{2}=\delta^{1 / 2}$, then the resulting 4-graph $\mathcal{H}^{\prime}$ has $\left(1-\delta_{2}\right) n$ vertices and number of edges at least

$$
\begin{aligned}
t^{4}(n)-\delta_{2} n d & >\frac{3}{32}\left(1-\delta-4 \delta_{2}\left(1-\delta_{1}\right)\right)\left(\begin{array}{l}
n \\
4
\end{array}\right) \\
& =\frac{3}{32}\left(1-\delta-4 \delta_{2}+4 \delta_{1} \delta_{2}\right)\left(\begin{array}{l}
n \\
4
\end{array}\right) \\
& >\frac{3}{32}\left(1-\delta-4 \delta_{2}+\left(2 \delta+6 \delta_{2}^{2}+6 \delta_{2}^{2} \delta-4 \delta_{2} \delta\right)\right)\left(\begin{array}{l}
n \\
4
\end{array}\right) \\
& =\frac{3}{32}(1+\delta)\left(1-4 \delta_{2}+6 \delta_{2}^{2}\right)\left(\begin{array}{l}
n \\
4
\end{array}\right) \\
& >\frac{3}{32}(1+\delta)\left(1-\delta_{2}\right)^{4}\left(\begin{array}{l}
n \\
4
\end{array}\right) \\
& >\frac{3}{32}(1+\delta)\left(\begin{array}{c}
\left(1-\delta_{2}\right) n \\
4
\end{array}\right) .
\end{aligned}
$$

By the result of Pikhurko [24] and Erdös-Simonovits supersaturation we conclude that $\mathcal{H}$ has at least $\delta^{\prime} n^{7}>\delta n^{4}$ copies of $P_{2}$ (for some fixed $\delta^{\prime}>0$ depending on $\delta$ ) and we are done. So we may assume that this process of removing vertices of degree less than $d$ terminates in at most $\delta_{2} n$ steps, and when it terminates we are left with a 4 -graph $\mathcal{H}^{\prime}$ on $n^{\prime}>\left(1-\delta_{2}\right) n$ vertices and minimum degree at least $d$.

Now suppose that we could prove that there is an edge of $\mathcal{H}^{\prime}$ that lies in at least $(2-$ $\varepsilon / 2)\left(n^{\prime} / 4\right)^{3}$ copies of $P_{2}$. Since $\delta \ll \varepsilon$, this is greater than $(2-\varepsilon)(n / 4)^{3}$ and we are done. If on the other hand $\mathcal{H}^{\prime}$ contains at least $2 \delta n^{\prime 4}$ copies of $P_{2}$, then again this is at least $\delta n^{4}$ and we are done. So if we could prove the result for $\mathcal{H}^{\prime}$ with $2 \delta, \varepsilon / 2$, then we could prove the result for $\mathcal{H}$ (with $\delta, \varepsilon$ ). Consequently, we may assume that $\mathcal{H}$ has minimum degree at least $\left(3 / 32-o_{\delta}(1)\right)\left(\begin{array}{l}n \\ 3\end{array}\right)=\left(1-o_{\delta}(1)\right)(n / 4)^{3}$.

If $\# P_{2} \geq \delta n^{4}$, then we are done so assume that $\# P_{2}<\delta n^{4}=\left(\delta / n^{3}\right) n^{7}$. Then by the Removal lemma, there is a set of at most $\delta n^{4}$ edges of $\mathcal{H}$ whose removal results in a 4 -graph $\mathcal{H}^{\prime}$ with no copies of $P_{2}$. Since $\left|\mathcal{H}^{\prime}\right|>t^{4}(n)-\delta n^{4}$, by Theorem 23 , we conclude that there is a partition of $\mathcal{H}^{\prime}$ (and also of $\mathcal{H}$ ) into four parts such that the number of edges intersecting some part in at least two points is $o_{\delta}\left(n^{4}\right)$. Now pick a partition $W \cup X \cup Y \cup Z$ of $\mathcal{H}$ that maximizes $e(W, X, Y, Z)$, the number of edges that intersect each part. We know that $e(W, X, Y, Z) \geq t^{4}(n)-o_{\delta}\left(n^{4}\right)$, and an easy calculation also shows that each of $W, X, Y, Z$ has size $n / 4 \pm o_{\delta}(n)$.

Let $B$ be the set of edges of $\mathcal{H}$ that intersect some part in at least two points. Let $G=\mathcal{H}-B$ 
be the set of edges of $\mathcal{H}$ that intersect each part. Let $M$ be the set of 4 -tuples which intersect each part and are not edges of $\mathcal{H}$. Then $\mathcal{H}-B \cup M=G \cup M$ is a 4-partite 4-graph with partition $W, X, Y, Z$, so it has at most $t^{4}(n)$ edges. We conclude that

$$
|M|<|B|<o_{\delta}\left(n^{4}\right)
$$

in particular $B \neq \emptyset$.

Claim. For every vertex $a$ of $\mathcal{H}$ we have $d_{M}(a)<\varepsilon_{1}(n / 4)^{3}$, where $\varepsilon_{1}=\varepsilon / 10^{5}$.

Proof of Claim. Suppose for contradiction that $d_{M}(a) \geq \varepsilon_{1}(n / 4)^{3}$ for some vertex $a$. Then

$$
\left(1-o_{\delta}(1)\right)(n / 4)^{3} \leq d_{\mathcal{H}}(a)=d_{G}(a)+d_{B}(a) \leq\left(1+o_{\delta}(1)\right)(n / 4)^{3}-\varepsilon_{1}(n / 4)^{3}+d_{B}(a) .
$$

We conclude that $d_{B}(a) \geq\left(\varepsilon_{1}-o_{\delta}(1)\right)(n / 4)^{3}>\left(\varepsilon_{1} / 2\right)(n / 4)^{3}$. Let $L=L(a)$ be the set of triples $\{b, c, d\}$ such that $a b c d \in B$. So $|L|=d_{B}(a)>\left(\varepsilon_{1} / 2\right)(n / 4)^{3}$. Partition $L=$ $L_{1} \cup L_{2} \cup L_{3}$, where $L_{i}$ consists of those triples that intersect precisely $i$ parts.

Case 1: $\left|L_{1}\right|>\left(\varepsilon_{1} / 10\right)(n / 4)^{3}$. Let us assume by symmetry that the number of triples $b c d \in L_{1}$ with $b, c, d \in W$ is at least $\left(\varepsilon_{1} / 40\right)(n / 4)^{3}$. For each choice of $(x, y, z) \in X \times Y \times Z$ with $a \neq x, y, z$, the three 4-tuples bxyz, cxyz, abcd form a potential copy of $P_{2}$. The number of such choices of $(\{b, c, d\}, x, y, z)$ is at least $\left(1-o_{\delta}(1)\right)\left(\varepsilon_{1} / 40\right)(n / 4)^{6}>\delta n^{6}$ so for at least half of these choices, one of the 4-tuples bxyz,cxyz must be in $M$. Each of these 4-tuples is counted at most $n^{2}$ times, since $a$ is fixed. We obtain the contradiction $\left(\varepsilon_{1} / 100 n^{2}\right)(n / 4)^{6}<$ $|M|=o_{\delta}\left(n^{4}\right)$. This concludes the proof in this case.

Case 2: $\left|L_{2}\right|>\left(3 \varepsilon_{1} / 10\right)(n / 4)^{3}$. Pick $b c d \in L_{2}$. There are $2 \times\left(\begin{array}{l}4 \\ 2\end{array}\right)=12$ possibilities for the way the points $b, c, d$ are distributed within the parts. Let us assume by symmetry that the number of triples $b c d \in L_{2}$ with $b, c \in W, d \in X$ is at least $\left(\varepsilon_{1} / 40\right)(n / 4)^{3}$. Now proceed exactly as in the proof of Case 1.

Case 3: $\left|L_{3}\right|>\left(\varepsilon_{1} / 10\right)(n / 4)^{3}$. Assume wlog that $a \in W$. Pick $b c d \in L_{3}$. There are 3 possibilities for the way the points $b, c, d$ are distributed within the parts (one point must be in $W$, the part containing $a$ ). Let us assume by symmetry that the number of triples $b c d \in L_{3}$ with $(b, c, d) \in W \times X \times Y$ is at least $\left(\varepsilon_{1} / 30\right)(n / 4)^{3}$. We may assume that $d_{G}(a) \geq\left(\varepsilon_{1} / 30\right)(n / 4)^{3}$ for otherwise we can move $a$ to $Z$ and increase $e(W, X, Y, Z)$ thereby contradicting the choice of the partition. Now pick $b c d \in L_{3}$ as above and $(x, y, z) \in$ $(X-\{c\}) \times(Y-\{d\}) \times Z$ with axyz $\in G$. For each choice of $(b, c, d, x, y, z)$ the three 4-tuples $a b c d, a x y z, b x y z$ form a copy of $P_{2}$. The number of such choices of $(b, c, d, x, y, z)$ 
is at least $\left(\varepsilon_{1} / 30\right)^{2}(n / 4)^{6}>\delta n^{6}$ so for at least half of these choices, one of the 4-tuples $b x y z \in M$. Each of these 4 -tuples is counted at most $n^{2}$ times, since $a$ is fixed. We obtain the contradiction $\left(\varepsilon_{1} / 30 n\right)^{2}(n / 4)^{6}<|M|=o_{\delta}\left(n^{4}\right)$. This concludes the proof of this case and the Claim.

Partition $B=B_{1} \cup B_{2}$, where $B_{2}$ consists of those edges of $B$ with exactly two points in one part, one point in a second part and one point in a third part (for example a $W W X Y$ edge would be in $B_{2}$ ). Suppose that $B_{1} \neq \emptyset$ and pick $e=a b c d \in B_{1}$. Some two points of $e$ must lie in the same part, so assume wlog that $a, b \in W$.

Let us first suppose that $c$ or $d$ is in $W$, say $c \in W$. For every $(x, y, z) \in X \times Y \times Z(x, y, z \neq$ $d$ ), we get three potential copies of $P_{2}$ of the form $w x y z, w^{\prime} x y z, e$ where $w, w^{\prime} \in\{a, b, c\}$. At least $(n / 4)^{3}$ of these potential copies of $P_{2}$ contains a 4 -tuple from $M$, otherwise we obtain $\left(2-o_{\delta}(1)\right)(n / 4)^{3}$ copies of $P_{2}$ containing $e$ and we are done. Each such 4 -tuple from $M$ is counted at most twice, so we obtain at least $(1 / 2)(n / 4)^{3} 4$-tuples from $M$ that contain some vertex in $\{a, b, c\}$. Consequently, there exists $w \in e$ with $d_{M}(w) \geq(1 / 6)(n / 4)^{3}$ and this contradicts the Claim.

We may therefore assume that $a, b \in W$ and $c, d$ lie in the same part different from $W$, say $c, d \in X$. There are at least $(\varepsilon / 3)(n / 4)^{3}$ choices $(x, y, z) \in(X-\{c, d\}) \times Y \times Z$ with $v x y z \in M$ for some $v \in\{a, b\}$ or there are at least $(\varepsilon / 3)(n / 4)^{3}$ choices $(w, y, z) \in(W-\{a, b\}) \times Y \times Z$ with vxyz $\in M$ for some $v \in\{c, d\}$. This is because otherwise $e$ would lie in at least $\left(2-o_{\delta}(1)-2 \varepsilon / 3\right)(n / 4)^{3}>(2-\varepsilon)(n / 4)^{3}$ copies of $P_{2}$. In either case, we conclude that there exists $v \in e$ with $d_{M}(v)>(\varepsilon / 6)(n / 4)^{3}$ thus contradicting the Claim.

We conclude from the arguments above that $B_{1}=\emptyset$. Pick $e=a b c d \in B_{2}$ and assume wlog that $a, b \in W, c \in X, d \in Y$. For $(x, y, z) \in(X-\{c\}) \times(Y-\{d\}) \times Z$, consider the following two potential copies of $P_{2}$ :

$$
e, b c d z, a x y z \quad e, a c d z, b x y z \text {. }
$$

The number of these potential copies of $P_{2}$ is twice the number of choices of $(x, y, z)$ and this is at least $\left(2-o_{\delta}(1)\right)(n / 4)^{3}$. At least $(\varepsilon / 2)(n / 4)^{3}$ of these potential $P_{2}$ 's has a 4 -tuple from $M$, otherwise we obtain at least $(2-\varepsilon)(n / 4)^{3}$ copies of $P_{2}$ containing $e$ and we are done. If for at least $(\varepsilon / 4)(n / 4)^{3}$ of these potential $P_{2}$ 's, the 4-tuple from $M$ is of the form $w x y z, w \in\{a, b\}$ (i.e., the third in the lists), then there exists $v \in\{a, b\}$ with $d_{M}(v) \geq(\varepsilon / 8)(n / 4)^{3}>\varepsilon_{1} n^{3}$ thereby contradicting the Claim. So for at least $(\varepsilon / 4)(n / 4)^{3}$ of these potential $P_{2}$ 's, the 4 - 
tuple from $M$ is of the form $w c d z, w \in\{a, b\}$ (i.e., the second in the lists). Each such 4-tuple from $M$ is counted at most $|X||Y|$ times, so there are at least $(\varepsilon / 4)(n / 4)^{3} /(|X||Y|)>(\varepsilon / 20) n$ 4-tuples from $M$ that intersect $e$ in three points.

Form the bipartite graph with parts $B=B_{2}$ and $M$ where $e \in B$ is adjacent to $f \in M$ if $|e \cap f|=3$. We have shown above that each $e \in B$ has degree at least $(\varepsilon / 20) n$. Since $|B|>|M|$, we conclude that there exists $f=a b c d \in M$ (with $(a, b, c, d) \in W \times X \times Y \times Z$ ) that is adjacent to at least $(\varepsilon / 20) n$ different $e$ 's from $B$. Assume wlog that at least $(\varepsilon / 80) n$ of these $e$ 's contain $a, b, c$. We may also assume wlog that at least $(\varepsilon / 240) n$ of these $e$ 's have their fourth vertex in the same part as $a$, namely $W$.

Now for each $j=1, \ldots,(\varepsilon / 240) n$, let $e_{j}=w_{j} a b c \in B$ with $w_{j} \in W$. For every $(x, y, z) \in$ $(X-\{b\}) \times(Y-\{c\}) \times Z$, consider the potential copy of $P_{2}$ given by $w_{j} x y z$, axyz, $e_{j}$. The number of choices for $(j, x, y, z)$ is at least $(\varepsilon / 240) n|X||Y||Z|>\left(4 \varepsilon / 10^{5}\right) n^{4}>2 \delta n^{4}$. If for at least half of these choices of $(j, x, y, z)$ the potential copy of $P_{2}$ is a real copy of $P_{2}$ in $\mathcal{H}$, then $\# P_{2} \geq \delta n^{4}$ and we are done. So we may assume that for at least half of the choices of $(j, x, y, z)$ (i.e. for at least $\left(2 \varepsilon / 10^{5}\right) n^{4}$ choices), the potential copy of $P_{2}$ referenced above has a 4-tuple $g \in M$. If at least half the time $a \notin g$, then we obtain the contradiction $\left(\varepsilon / 10^{5}\right) n^{4} \leq|M|=o_{\delta}\left(n^{4}\right)$. So at least half the time $a \in g$. Each such $g$ containing $a$ is counted at most $n$ times (once for each $w_{j}$ ), so we obtain $d_{M}(a)>\left(\varepsilon / 10^{5}\right) n^{3} \geq \varepsilon_{1} n^{3}$. This contradicts the Claim and completes the proof of the theorem.

\subsection{Counting $P_{3}$ 's}

Recall that $c\left(n, P_{3}\right)=2(n / 2)^{3}-\Theta\left(n^{2}\right)$. Theorem 9 for $l=3$ follows from the following result.

Theorem 24. For every $\varepsilon>0$ there exists $\delta>0$ and $n_{0}$ such that the following holds for $n>n_{0}$. Every $n$ vertex 4-graph with $d^{4}(n)+1$ edges contains either

- an edge that lies in at least $(2-\varepsilon)(n / 2)^{3}$ copies of $P_{3}$, or

- at least $\delta n^{4}$ copies of $P_{3}$.

Proof of Theorem 9 for $l=3$. Remove $q-1$ edges from $\mathcal{H}$ and apply Theorem 24 . If we find $\delta n^{4}$ copies of $P_{3}$, then since $q<\delta n$, the number of copies is much larger than 
$q(1-\varepsilon) c\left(n, P_{3}\right)$ and we are done. Consequently, we find an edge $e_{1}$ in at least $(2-\varepsilon)(n / 2)^{3}>$ $(1-\varepsilon) c\left(n, P_{3}\right)$ copies of $P_{3}$. Now remove $q-2$ edges from $\mathcal{H}-e_{1}$ and repeat this argument to obtain $e_{2}$. In this way we obtain edges $e_{1}, \ldots, e_{q}$ as required.

The bound is sharp due to the following construction. Add a collection of $q$ pairwise disjoint edges within one part of $D^{4}(n)$. It is easy to see that each added edge lies in $2(n / 2)^{3}+O\left(n^{2}\right)$ copies of $P_{3}$ and clearly no copy of $P_{3}$ contains two of the new edges. Consequently, the copies of $P_{3}$ are counted exactly once.

We will need the following stability theorem for $P_{3}$ proved by Füredi-Pikhurko-Simonovits $[9]$

Theorem 25. ( $P_{3}$ stability [9]) Let $\mathcal{H}$ be a 4-graph with $n$ vertices and $d^{4}(n)-o\left(n^{4}\right)$ edges that contains no copy of $P_{3}$. Then there is a partition of the vertex set of $\mathcal{H}$ into $X \cup Y$ so that the number of edges that intersect some part in 0, 1, 3 or 4 points is o( $\left.n^{4}\right)$. In other words, $\mathcal{H}$ can be obtained from $D^{4}(n)$ by adding and deleting a set of $o\left(n^{4}\right)$ edges.

Proof of Theorem 24. Given $\varepsilon$ let $0<\delta \ll \varepsilon$. Write $o_{\delta}(1)$ for any function that approaches zero as $\delta$ approaches zero and moreover, $o_{\delta}(1) \ll \varepsilon$. Let $n$ be sufficiently large and let $\mathcal{H}$ be an $n$ vertex 4-graph with $d^{4}(n)+1$ edges. Write $\# P_{3}$ for the number of copies of $P_{3}$ in $\mathcal{H}$.

As in the proof of Theorem 22 (just replacing $3 / 32$ by $3 / 8$ ), we may assume that $\mathcal{H}$ has minimum degree at least $d=(3 / 8)\left(1-o_{\delta}(1)\right)\left(\begin{array}{l}n \\ 3\end{array}\right)$

If $\# P_{3} \geq \delta n^{4}$, then we are done so assume that $\# P_{3}<\delta n^{4}=\left(\delta / n^{3}\right) n^{7}$. Then by the Removal lemma, there is a set of at most $\delta n^{4}$ edges of $\mathcal{H}$ whose removal results in a 4 graph $\mathcal{H}^{\prime}$ with no copies of $P_{3}$. Since $\left|\mathcal{H}^{\prime}\right|>d^{4}(n)-\delta n^{4}$, by Theorem 25 , we conclude that there is a partition of $\mathcal{H}^{\prime}$ (and also of $\mathcal{H}$ ) into two parts such that the number of edges intersecting some part in $0,1,3$, or 4 points is $o_{\delta}\left(n^{4}\right)$. Now pick a partition $X \cup Y$ of $\mathcal{H}$ that maximizes $e(X, Y)$, the number of edges that intersect each part in two points. We know that $e(X, Y) \geq d^{4}(n)-o_{\delta}\left(n^{4}\right)$, and an easy calculation also shows that each of $X, Y$ has size $n / 2 \pm o_{\delta}(n)$.

Let $B$ be the set of edges of $\mathcal{H}$ that intersect some part in $0,1,3$ or 4 points. Let $G=\mathcal{H}-B$ be the set of edges of $\mathcal{H}$ that intersect each part in two points. Let $M$ be the set of 4 -tuples which intersect each part in two points and are not edges of $\mathcal{H}$. Then $\mathcal{H}-B \cup M=G \cup M$ 
is a 4-graph with $(2,2)$-partition $X \cup Y$, so it has at most $d^{4}(n)$ edges. We conclude that

$$
|M|<|B|<o_{\delta}\left(n^{4}\right)
$$

in particular $B \neq \emptyset$.

Claim. For every vertex $a$ of $\mathcal{H}$ we have $d_{M}(a)<\varepsilon_{1}(n / 2)^{3}$, where $\varepsilon_{1}=\varepsilon / 10^{6}$.

Proof of Claim. Suppose for contradiction that $d_{M}(a) \geq \varepsilon_{1}(n / 2)^{3}$ for some vertex $a$. Then

$$
\left(1 / 2-o_{\delta}(1)\right)(n / 2)^{3} \leq d_{\mathcal{H}}(a)=d_{G}(a)+d_{B}(a) \leq\left(1 / 2+o_{\delta}(1)\right)(n / 2)^{3}-\varepsilon_{1}(n / 2)^{3}+d_{B}(a) .
$$

We conclude that $d_{B}(a) \geq\left(\varepsilon_{1}-o_{\delta}(1)\right)(n / 2)^{3}>\left(\varepsilon_{1} / 2\right)(n / 2)^{3}$. Let $L=L(a)$ be the set of triples $\{b, c, d\}$ such that $a b c d \in B$. So $|L|=d_{B}(a)>\left(\varepsilon_{1} / 2\right)(n / 2)^{3}$. Assume wlog that $a \in X$. Partition $L=L_{X X X} \cup L_{X X Y} \cup L_{Y Y Y}$, where $L_{X^{i} Y^{3-i}}$ consists of those triples that intersect $X$ in precisely $i$ points (note that $L_{X Y Y}=\emptyset$ by definition of $B$ ).

Case 1: $\left|L_{X X X}\right|>\left(\varepsilon_{1} / 6\right)(n / 2)^{3}$ or $\left|L_{Y Y Y}\right|>\left(\varepsilon_{1} / 6\right)(n / 2)^{3}$. Let us first assume that $\left|L_{X X X}\right|>$ $\left(\varepsilon_{1} / 6\right)(n / 2)^{3}$. For each $b c d \in L_{X X X}$ with $e=a b c d$ and $\left(x,\left\{y, y^{\prime}\right\}\right) \in(X-e) \times\left(\begin{array}{c}Y \\ 2\end{array}\right)$, the four 4 -tuples $b x y y^{\prime}, c x y y^{\prime}, d x y y^{\prime}, e$ form a potential copy of $P_{3}$. The number of such choices of $\left(e, x,\left\{y, y^{\prime}\right\}\right)$ is at least $\left(\varepsilon_{1} / 13\right)(n / 2)^{6}>\delta n^{6}$ so for at least half of these choices, one of the 4-tuples $b x y y^{\prime}, c x y y^{\prime}, d x y y^{\prime}$ must be in $M$. Each of these 4 -tuples in $M$ is counted at most $|X|^{2}<n^{2}$ times, since $a$ is fixed. We obtain the contradiction $\left(\varepsilon_{1} / 13 n^{2}\right)(n / 2)^{6}<|M|=$ $o_{\delta}\left(n^{4}\right)$. If $\left|L_{Y Y Y}\right|>\left(\varepsilon_{1} / 6\right)(n / 2)^{3}$, then the same proof works by replacing $\left(x,\left\{y, y^{\prime}\right\}\right)$ with $\left(\left\{x, x^{\prime}\right\}, y\right) \in\left(\begin{array}{c}X \\ 2\end{array}\right) \times(Y-e)$. This concludes the proof in this case.

Case 2: $\left|L_{X X Y}\right|>\left(\varepsilon_{1} / 6\right)(n / 2)^{3}$. We may assume that $d_{G}(a) \geq\left|L_{X X Y}\right|$ for otherwise we can move $a$ to $Y$ and increase $e(X, Y)$ thereby contradicting the choice of the partition. Pick $b c d \in L_{X X Y}$ with $b, c \in X$ and $d \in Y$. Consider $x, y, y^{\prime}$ with $x \in X-e, y, y^{\prime} \in Y-e$ and $a x y y^{\prime} \in G$. For each choice of $\left(e, x,\left\{y, y^{\prime}\right\}\right)$ the four 4-tuples $b c d y, b c d y^{\prime}, e, a x y y^{\prime}$ form a copy of $P_{3}$. The number of such choices of $\left(e, x,\left\{y, y^{\prime}\right\}\right)$ is at least

$$
d_{G}(a)\left|L_{X X Y}\right| \geq\left|L_{X X Y}\right|^{2}>2\left(\varepsilon_{1} / 10^{5}\right)^{2} n^{6}>2 \delta n^{6}
$$

so for at least half of these choices, one of the 4-tuples $b c d y, b c d y^{\prime} \in M$. Each of these 4-tuples of $M$ is counted at most $n^{2}$ times, since $a$ is fixed. We obtain the contradiction $\left(\varepsilon_{1} / 10^{5}\right)^{2} n^{4}<|M|=o_{\delta}\left(n^{4}\right)$. This concludes the proof of this case and the Claim.

Partition $B=B_{1} \cup B_{2}$, where $B_{2}$ consists of those edges of $B$ that intersect both parts in an odd number of points. Suppose that $B_{1} \neq \emptyset$, pick $e=a b c d \in B_{1}$ and assume wlog that 
$e \subset X$. For every $\left(x,\left\{y, y^{\prime}\right\}\right) \in(X-e) \times\left(\begin{array}{c}Y \\ 2\end{array}\right)$, we get four potential copies of $P_{3}$ of the form $w_{1} x y y^{\prime}, w_{2} x y y^{\prime}, w_{3} x y y^{\prime}, e$ where $\left\{w_{1}, w_{2}, w_{3}\right\} \subset e$ and $w_{i} \neq w_{j}$. At least $(\varepsilon / 2)(n / 2)^{3}$ of these potential copies of $P_{3}$ contains a 4-tuple from $M$, otherwise we obtain

$$
4(|X|-4)\left(\begin{array}{c}
|Y| \\
2
\end{array}\right)-(\varepsilon / 2)(n / 2)^{3}=\left(2-o_{\delta}(1)-\varepsilon / 2\right)(n / 2)^{3}>(2-\varepsilon)(n / 2)^{3}
$$

copies of $P_{3}$ containing $e$ and we are done. Each such 4-tuple from $M$ contains some point of $e$ so there exists $w \in e$ with $d_{M}(w) \geq(\varepsilon / 8)(n / 2)^{3}>\varepsilon_{1}(n / 2)^{3}$ and this contradicts the Claim.

We conclude the $B_{1}=\emptyset$. Pick $e=a b c d \in B_{2}$ and assume wlog that $a, b, c \in X, d \in Y$. For $\left(x,\left\{y, y^{\prime}\right\}\right) \in(X-e) \times\left(\begin{array}{c}Y-e \\ 2\end{array}\right)$, consider the following types of potential copies of $P_{3}$ :

Type 1: $x y y^{\prime} a, x y y^{\prime} b, x y y^{\prime} c, e$

Type 2: $e, a b d y, a b d y^{\prime}, x c y y^{\prime} ; \quad e, a c d y, a c d y^{\prime}, x b y y^{\prime} ; \quad e, b c d y, b c d y^{\prime}, x a y y^{\prime}$.

At least $(\varepsilon / 2)(n / 2)^{3}$ of these potential copies of $P_{3}$ contains a 4 -tuple from $M$, otherwise we obtain at least $(2-\varepsilon)(n / 2)^{2}$ copies of $P_{3}$ containing $e$ (as in (9)) and we are done. Suppose that at least half the time, the 4 -tuple from $M$ is in one of the Type 1 copies, or the last 4 -tuple in one of the type two copies (i.e., $\left.x c y y^{\prime}, x b y y^{\prime}, x a y y^{\prime}\right)$. Each such 4-tuple is counted at most twice, and so we obtain at least $(\varepsilon / 8)(n / 2)^{3} 4$-tuples of $M$ that intersect $e$. We conclude that there exists $w \in e$ with $d_{M}(w) \geq(\varepsilon / 32)(n / 2)^{3}>\varepsilon_{1}(n / 2)^{3}$ and this contradicts the Claim.

We may therefore assume that for at least $(\varepsilon / 4)(n / 2)^{3}$ of these potential copies of $P_{3}$, the 4-tuple from $M$ is one of the two middle ones of the Type 2 copies, and so it intersects $e$ in three points. Each such 4 -tuple is counted at most $|X||Y|<\left(1+o_{\delta}(1)\right)(n / 2)^{2}$ times, so we obtain at least $(\varepsilon / 10) n$ 4-tuples from $M$ that intersect $e$ in three points. We have argued that for every $e \in B_{2}=B$, there are at least $(\varepsilon / 10) n$ different $f \in M$ for which $|e \cap f|=3$. Since $|B|>|M|$, we conclude that there exists $f^{\prime} \in M$ with at least $(\varepsilon / 10) n$ different $e^{\prime} \in B_{2}$ such that $\left|e^{\prime} \cap f^{\prime}\right|=3$. At least $(\varepsilon / 40) n$ of these $e^{\prime}$ s intersect $f$ in the same three points. Consequently, we may assume wlog that there are $a, b \in X, d \in Y$ and $x_{1}, \ldots, x_{t} \in X$ with $t=(\varepsilon / 40) n$ such that $e_{i}=a b x_{i} d \in B$.

Fix $i$, set $e=e_{i}$ and consider the Type 1 potential copies of $P_{3}$ referenced in the notation above with $c=x_{i}$, i.e., consider $x y y^{\prime} a, x y y^{\prime} b, x y y^{\prime} c, e$. Recall that there are at least (1$\left.o_{\delta}(1)\right)|X|\left(\begin{array}{c}|Y| \\ 2\end{array}\right)>(1 / 20) n^{3}$ such copies. If at least $\varepsilon n^{3}$ of these potential copies of $P_{3}$ have a 4-tuple from $M$, then we find a vertex $w \in\left\{a, b, x_{i}\right\}$ with $d_{M}(w)>(\varepsilon / 4) n^{3}>\varepsilon_{1} n^{3}$ and this 
contradicts the Claim. We conclude that each $e_{i}=a b c x_{i}$ lies in at least $(1 / 20-\varepsilon) n^{3}>\varepsilon n^{3}$ copies of $P_{3}$, and these copies are clearly distinct for distinct $i$. Altogether we therefore have $\# P_{3} \geq t \varepsilon n^{3}=\left(\varepsilon^{2} / 40\right) n^{4}>\delta n^{4}$ and we are done.

\subsection{Counting $P_{4}$ 's}

Recall that $c\left(n, P_{4}\right)=(4+o(1))\left(\begin{array}{c}n / 2 \\ 3\end{array}\right)=\Theta\left(n^{3}\right)$. Theorem 9 for $l=4$ follows from the following result.

Theorem 26. For every $\varepsilon>0$ there exists $\delta>0$ and $n_{0}$ such that the following holds for $n>n_{0}$. Every $n$ vertex 3-graph with $b^{4}(n)+1$ edges contains either

- an edge that lies in at least $(1-\varepsilon) c\left(n, P_{4}\right)$ copies of $P_{4}$, or

- at least $\delta n^{4}$ copies of $P_{4}$.

Proof of Theorem 9 for $l=4$. Remove $q-1$ edges from $\mathcal{H}$ and apply Theorem 26 . If we find $\delta n^{4}$ copies of $P_{4}$, then since $q<\delta n$, the number of copies is much larger than $q(1-\varepsilon) c\left(n, P_{4}\right)$ and we are done. Consequently, we find an edge $e_{1}$ in at least $(1-\varepsilon) c\left(n, P_{4}\right)$ copies of $P_{4}$. Now remove $q-2$ edges from $\mathcal{H}-e_{1}$ and repeat this argument to obtain $e_{2}$. In this way we obtain edges $e_{1}, \ldots, e_{q}$ as required.

The result is asymptotically tight as we can add $q$ pairwise disjoint 4 -tuples to $B^{4}(n)$, each intersecting both parts in two points.

We need the following stability result proved in [8].

Theorem 27. ( $P_{4}$ stability [8]) Let $\mathcal{H}$ be a 4-graph with $n$ vertices and $b^{4}(n)-o\left(n^{4}\right)$ edges that contains no copy of $P_{4}$. Then there is a partition of the vertex set of $\mathcal{H}$ into $X \cup Y$ so that the number of edges that intersect $X$ or $Y$ in an even number of points is o( $\left.n^{4}\right)$. In other words, $\mathcal{H}$ can be obtained from $B^{4}(n)$ by adding and deleting a set of o $\left(n^{4}\right)$ edges.

Proof of Theorem 26. Given $\varepsilon$ let $0<\delta \ll \varepsilon$. Write $o_{\delta}(1)$ for any function that approaches zero as $\delta$ approaches zero and moreover, $o_{\delta}(1) \ll \varepsilon$. Let $n$ be sufficiently large and let $\mathcal{H}$ be an $n$ vertex 4 -graph with $b^{4}(n)+1$ edges. Write $\# P_{4}$ for the number of copies of $P_{4}$ in $\mathcal{H}$. 
As in the proof of Theorem 22 (just replacing $3 / 32$ by $1 / 2$ ), we may assume that $\mathcal{H}$ has minimum degree at least $d=\left(1 / 2-o_{\delta}(1)\right)\left(\begin{array}{l}n \\ 3\end{array}\right)$.

If $\# P_{4} \geq \delta n^{4}$, then we are done so assume that $\# P_{4}<\delta n^{4}$. Then by the Removal lemma, there is a set of at most $\delta n^{4}$ edges of $\mathcal{H}$ whose removal results in a 4 -graph $\mathcal{H}^{\prime}$ with no copies of $P_{4}$. Since $\left|\mathcal{H}^{\prime}\right|>b^{4}(n)-\delta n^{4}$, by Theorem 27 , we conclude that there is a partition of $\mathcal{H}^{\prime}$ (and also of $\mathcal{H}$ ) into two parts such that the number of edges intersecting some part in an even number of points is $o_{\delta}\left(n^{4}\right)$. Now pick a partition $X \cup Y$ of $\mathcal{H}$ that maximizes $e(X, Y)$, the number of edges that intersect each part in an odd number of points. We know that $e(X, Y) \geq b^{4}(n)-o_{\delta}\left(n^{4}\right)$, and an easy calculation also shows that each of $X, Y$ has size $n / 2 \pm o_{\delta}(n)$.

Let $B$ be the set of edges of $\mathcal{H}$ that intersect one (and therefore both) of $X, Y$ in an even number of points. Let $G=\mathcal{H}-B$ be the set of edges of $\mathcal{H}$ that intersect both $X, Y$ in an odd number of points. Let $M$ be the set of 4-tuples which intersect both parts in an odd number of points and are not edges of $\mathcal{H}$. Then $\mathcal{H}-B \cup M=G \cup M$ is an odd 4-graph with partition $X, Y$, so it has at most $b^{4}(n)$ edges. We conclude that

$$
|M|<|B|<o_{\delta}\left(n^{4}\right)
$$

in particular $B \neq \emptyset$. Let $B_{X^{i} Y^{4-i}}\left(\mathcal{H}_{X^{i} Y^{4-i}}\right)$ be the set of edges in $B(\mathcal{H})$ with exactly $i$ points in $X$. Let

$$
\varepsilon_{1}=\min \left\{\varepsilon / 200, \varepsilon^{2} / 10^{4}, \varepsilon^{3} / 10^{3}\right\}
$$

Claim. For every vertex $a$ of $\mathcal{H}$ we have $d_{M}(a)<\varepsilon_{1} n^{3}$.

Proof of Claim. Suppose for a contradiction that $d_{M}(a)>\varepsilon_{1} n^{3}$. Since

$$
\left(1 / 2-o_{\delta}(1)\right)\left(\begin{array}{l}
n \\
3
\end{array}\right) \leq d_{\mathcal{H}}(a)=d_{G}(a)+d_{B}(a) \leq\left(\left(1 / 2+o_{\delta}(1)\right)\left(\begin{array}{l}
n \\
3
\end{array}\right)-d_{M}(a)\right)+d_{B}(a)
$$

we conclude that $d_{B}(a)>d_{M}(a)-o_{\delta}\left(n^{3}\right)>\left(\varepsilon_{1} / 2\right) n^{3}$. Assume wlog that $a \in X$. Then $d_{B}(a)=d_{B_{X X X X}}(a)+d_{B_{X X Y Y}}(a)$.

Case 1. $d_{B_{X X X X}}(a) \geq\left(\varepsilon_{1} / 4\right) n^{3}$

We may assume that $d_{G}(a) \geq d_{B_{X X X X}}(a)$ otherwise moving $a$ from $X$ to $Y$ increases $e(X, Y)$ and contradicts the choice of the partition. Pick $e=a b c d \in B_{X X X X}$ and $f=a u v w \in G$. The number of choices for $(e, f)$ is at least $d_{B_{X X X X}}(a)^{2} \geq\left(\varepsilon_{1} / 4\right)^{4} n^{6}$. For each such $(e, f)$, consider the five 4-tuples

uvwb, uvwc, uvwd, $f, \quad e$. 
This gives at least $\left(\varepsilon_{1}^{2} / 16\right) n^{6}>2 \delta n^{6}$ potential copies of $P_{4}$ so at least $\left(\varepsilon_{1}^{2} / 32\right) n^{6}$ of them have a 4 -tuple not in $\mathcal{H}$. Since $e, f \in \mathcal{H}$, the absent 4-tuple is of the form uvwz where $z \in\{b, c, d\}$. Notice that $u v w z \in M$ since auvw $\in G$ and $a, z \in X$. Each such 4-tuple of $M$ is counted at most $3 n^{2}$ times, as there are at most three choices for $|e \cap f|$ and $\left(\begin{array}{c}|X| \\ 2\end{array}\right)<n^{2}$ choices for the two vertices of $e-f-\{a\}$. This yields the contradiction $\left(\varepsilon_{1}^{2} / 32\right) n^{6} /\left(3 n^{2}\right)<|M|=o_{\delta}\left(n^{4}\right)$.

Case 2. $d_{B_{X X Y Y}}(a) \geq\left(\varepsilon_{1} / 4\right) n^{3}$

Suppose that $d_{\mathcal{H}_{X Y Y Y}}(a) \geq\left(\varepsilon_{1} / 2\right) n^{3}$. Then pick $e=a b c d \in B_{X X Y Y}$ and $f=a u v w \in \mathcal{H}_{X Y Y Y}$ (so $u, v, w \in Y$ ) with $|e \cap f|=1$. The number of such pairs $(e, f)$ is at least $\left(\varepsilon_{1}^{2} / 10\right) n^{6}$. For each such $(e, f)$, consider the potential copy of $P_{4}$ given by

$$
b c d u, \quad b c d v, \quad b c d w, \quad e, \quad f \text {. }
$$

Since $\left(\varepsilon_{1}^{2} / 10\right) n^{6}>2 \delta n^{6}$ at least half of them have a 4 -tuple from $M$. Each such 4 -tuple is counted at most $3 n^{2}$ times, so we obtain the contradiction $\left(\varepsilon_{1}^{2} / 20\right) n^{6} /\left(3 n^{2}\right) \leq|M|=o_{\delta}\left(n^{4}\right)$.

We may therefore assume that

(i) $d_{\mathcal{H}_{X Y Y Y}}(a)<\left(\varepsilon_{1} / 2\right) n^{3}$ and

(ii) $d_{B_{X X X X}}(a)<\left(\varepsilon_{1} / 4\right) n^{3}$.

Define $L(a)=\{b c d: a b c d \in \mathcal{H}\}$, so $|L(a)|=d_{\mathcal{H}}(a) \geq\left(1 / 2-o_{\delta}(1)\right)\left(\begin{array}{l}n \\ 3\end{array}\right)$. Consider the partition

$$
L(a)=L_{X X X} \cup L_{X X Y} \cup L_{X Y Y} \cup L_{Y Y Y}
$$

where the subscripts have the obvious meaning. Then (i) and (ii) translate to

$$
\left|L_{Y Y Y}\right|+\left|L_{X X X}\right|<\varepsilon_{1} n^{3} .
$$

For $(u, v) \in X \times Y$, let $d_{L}(u v)$ be the number of $w$ such that $u v w \in L(a)$. Then

$$
\begin{aligned}
\sum_{(u, v) \in X \times Y} d_{L}(u, v) & =2\left(\left|L_{X X Y}\right|+\left|L_{X Y Y}\right|\right) \\
& =2\left(|L(a)|-\left|L_{Y Y Y}\right|-\left|L_{X X X}\right|\right) \\
& >2\left(|L(a)|-\varepsilon_{1} n^{3}\right) \\
& \geq 2\left(1 / 2-o_{\delta}(1)\right)\left(\begin{array}{l}
n \\
3
\end{array}\right)-2 \varepsilon_{1} n^{3} \\
& \geq\left(1-13 \varepsilon_{1}-o_{\delta}(1)\right)\left(\begin{array}{l}
n \\
3
\end{array}\right)
\end{aligned}
$$


Consequently, there exists $(b, c) \in X \times Y$ such that

$$
d_{L}(b c)>\frac{\left(1-13 \varepsilon_{1}-o_{\delta}(1)\right)\left(\begin{array}{l}
n \\
3
\end{array}\right)}{\left(1 / 4+o_{\delta}(1)\right) n^{2}}>\left(2 / 3-9 \varepsilon_{1}\right) n .
$$

We conclude that there exists $S \subset X, T \subset Y$ such that

$$
\min \{|S|,|T|\} \geq\left(2 / 3-9 \varepsilon_{1}-1 / 2-o_{\delta}(1)\right) n>\left(1 / 6-10 \varepsilon_{1}\right) n
$$

and abcd $\in \mathcal{H}$ for every $d \in S \cup T$. Now pick $s_{1}, s_{2}, s_{3} \in S$ and $t \in T$ and consider the potential $P_{4}$

$$
a b c s_{1}, \quad a b c s_{2}, \quad a b c s_{3}, \quad a b c t, \quad s_{1} s_{2} s_{3} t .
$$

The number of choice for $\left(\left\{s_{1}, s_{2}, s_{3}\right\}, t\right)$ is at least $\left(\begin{array}{c}|S| \\ 3\end{array}\right)|T|>10^{-4} n^{4}$. If for at least half of these choices of $\left(\left\{s_{1}, s_{2}, s_{3}\right\}, t\right)$ we get a copy of $P_{4}$ in $\mathcal{H}$ as shown above, then $\# P_{4}>$ $(1 / 2) 10^{-4} n^{4}>\delta n^{4}$, a contradiction. So for at least half of the choices, one of the 4-tuples in (11) is not in $\mathcal{H}$. By definition of $S$ and $T$, the first four are in $\mathcal{H}$, so the last one is in $M$. This is counted exactly once, so we obtain the contradiction $(1 / 2) 10^{-4} n^{4}<|M|=o_{\delta}\left(n^{4}\right)$. This completes the proof of the Claim.

Partition $B=B_{1} \cup B_{X X Y Y}$ where

$$
B_{1}=B_{X X X X} \cup B_{Y Y Y Y}
$$

Case 1. $\left|B_{1}\right| \geq \varepsilon|B|$.

Pick $e=a b c d \in B_{1}$, and assume wlog that $e \in B_{X X X X}$. Let $e^{\prime} \subset e$ with $\left|e^{\prime}\right|=3$. Assume wlog that $e^{\prime}=b c d$. Let $\left\{y_{1}, y_{2}, y_{3}\right\} \in\left(\begin{array}{l}Y \\ 3\end{array}\right)$ and consider the five 4 -tuples

$$
b c d y_{1}, \quad b c d y_{2}, \quad b c d y_{3}, \quad e, \quad a y_{1} y_{2} y_{3} .
$$

These 4-tuples from a potential copy of $P_{4}$. The number of choices for $\left(e^{\prime},\left\{y_{1}, y_{2}, y_{3}\right\}\right)$ is at least $4\left(1-o_{\delta}(1)\right)\left(\begin{array}{c}n / 2 \\ 3\end{array}\right)$. For at least $2 \varepsilon\left(\begin{array}{c}n / 2 \\ 3\end{array}\right)$ of these choices, one of the 4 -tuples above must not be in $\mathcal{H}$, otherwise $\# P_{4} \geq 4\left(1-o_{\delta}(1)\right)\left(\begin{array}{c}n / 2 \\ 3\end{array}\right)-2 \varepsilon\left(\begin{array}{c}n / 2 \\ 3\end{array}\right)>(1-\varepsilon) c\left(n, P_{4}\right)$ and we are done. If for at least $\varepsilon\left(\begin{array}{c}n / 2 \\ 3\end{array}\right)$ of these choices, the missing 4-tuple is the last one in the list, then we obtain $d_{M}(x)>(\varepsilon / 4)\left(\begin{array}{c}n / 2 \\ 3\end{array}\right)>\varepsilon_{1} n^{3}$ for some $x \in e\left(\right.$ since $\left.\varepsilon_{1} \leq \varepsilon / 200\right)$. This contradicts the Claim. We may therefore assume that for at least $\varepsilon\left(\begin{array}{c}n / 2 \\ 3\end{array}\right)$ of these choices, the 4-tuple from $M$ has exactly three points in $e$. Each such 4-tuple is counted at most $\left(\begin{array}{c}|Y| \\ 2\end{array}\right)$ times giving at least $(\varepsilon / 7) n$ 4-tuples from $M$ with three points in $e$. 
We have argued above that for every $e \in B_{1}$, there are more than $(\varepsilon / 7) n$ different $f \in M$ with $|e \cap f|=3$. Form the bipartite graph with parts $B_{1}, M$, where $e \in B_{1}$ is adjacent to $f \in M$ if $|e \cap f|=3$. Then each vertex in $B_{1}$ has degree more than $(\varepsilon / 7) n$, and since $\left|B_{1}\right| \geq \varepsilon|B|>\varepsilon|M|$, we conclude that there exists $f \in M$ that is adjacent to more than $\left|B_{1}\right|(\varepsilon / 7) n /|M|>\left(\varepsilon^{2} / 7\right) n$ different $e \in B_{1}$. Consequently, there exist $a, b, c$ such that $d_{B_{1}}(a b c)>\left(\varepsilon^{2} / 7\right) n$. Assume wlog that $a, b, c \in X$.

For each choice of $d$ with $e=a b c d \in B_{1}$ and $\left\{y_{1}, y_{2}, y_{3}\right\} \in\left(\begin{array}{l}Y \\ 3\end{array}\right)$ five 4-tuples $y_{1} y_{2} y_{3} x$ where $x \in e$ together with $e$ form a potential copy of $P_{4}$. The number of choices for $\left(d,\left\{y_{1}, y_{2}, y_{3}\right\}\right)$ is at least $d_{B_{1}}(a b c)\left(\begin{array}{c}|Y| \\ 3\end{array}\right)>6 \varepsilon_{1} n^{4}>2 \delta n^{4}$ (since $\varepsilon_{1} \leq \varepsilon^{2} / 10^{4}$ ). If for at least half of them, we get a copy of $P_{4}$ in $\mathcal{H}$, then $\# P_{4}>\delta n^{4}$ and we are done. So for at least $3 \varepsilon_{1} n^{4}$ of the choices, one of the five 4 -tuples is not in $\mathcal{H}$. If for at least $\varepsilon_{1} n^{4}$ choices the missing 4 -tuple is of the form $y_{1} y_{2} y_{3} d$, then we obtain the contradiction $\varepsilon_{1} n^{4} \leq|M|=o_{\delta}\left(n^{4}\right)$. So for at least $2 \varepsilon_{1} n^{4}$ choices the missing 4 -tuple is of the form $y_{1} y_{2} y_{3} x, x \neq d$. Each such missing 4-tuple is counted at most $|X|<n$ times. We conclude that there exists $x \in e$ with $d_{M}(x)>2 \varepsilon_{1} n^{4} / n>\varepsilon_{1} n^{3}$. This contradicts the Claim and completes the proof in this case.

Case 2. $\left|B_{1}\right|<\varepsilon|B|$.

In this case we have $\left|B_{X X Y Y}\right| \geq(1-\varepsilon)|B|$. Partition $B_{X X Y Y}=B_{2} \cup B_{3}$ where

$$
B_{2}=\left\{e \in B_{X X Y Y}: d_{M}\left(e^{\prime}\right)>(1-\varepsilon)(n / 2) \text { for every } e^{\prime} \subset e \text { with }\left|e^{\prime}\right|=3\right\} .
$$

Suppose that $\left|B_{2}\right| \geq(1-\varepsilon)\left|B_{X X Y Y}\right|$. Then we count 4-tuples of $M$ from sets in $B_{2}$. For each set in $B_{2}$, there are four choices for $e^{\prime} \subset e$ with $\left|e^{\prime}\right|=3$, and given $e^{\prime}$, there are $(1-\varepsilon)(n / 2)$ 4-tuples of $M$ containing $e^{\prime}$. Each 4 -tuple from $M$ is counted at most $3 \max \{|X|,|Y|\}$ times. This gives the contradiction

$$
|M| \geq \frac{4(1-\varepsilon)(n / 2)\left|B_{2}\right|}{3 \max \{|X|,|Y|\}}>\frac{4(1-2 \varepsilon)\left|B_{2}\right|}{3} \geq \frac{4(1-2 \varepsilon)(1-\varepsilon)^{2}|B|}{3}>|B|>|M| .
$$

We may therefore suppose that $\left|B_{3}\right|>\varepsilon\left|B_{X X Y Y}\right|>(\varepsilon / 2)|B|$. We may also assume that no edge of $B$ lies in at least $(1-\varepsilon) c\left(n, P_{4}\right)$ copies of $P_{4}$, otherwise we are done. Using this observation we conclude that we have at least $(\varepsilon / 4) n 4$-tuples in $M$. To see this we pick an edge $e \in B$ and consider potential copies of $P_{4}$ containing $e$. We know that at least $(\varepsilon / 2) c\left(n, P_{4}\right)$ of these potential copies have a 4-tuple from $M$, for otherwise $e$ lies in at least $(1-\varepsilon) c\left(n, P_{4}\right)$ copies of $P_{4}$. Each such 4-tuple is counted at $\operatorname{most} \max \left\{\left(\begin{array}{c}|X| \\ 2\end{array}\right),\left(\begin{array}{c}|Y| \\ 2\end{array}\right)\right\}$ times. So we may assume that

$$
|B|>|M|>(\varepsilon / 4) n
$$


Now pick an edge $e=a b c d \in B_{3}$. By definition of $B_{3}$, there exists $e^{\prime}=b c d \subset e$ with $d_{M}(b c d) \leq(1-\varepsilon) n / 2$. Assume wlog that $b \in X, c, d \in Y$. Then there is a set $Y^{\prime} \subset Y$ such that $b c d y \in \mathcal{H}$ for every $y \in Y^{\prime}$ and

$$
\left|Y^{\prime}\right| \geq|Y|-2-d_{M}(b c d) \geq\left(1-o_{\delta}(1)\right)(n / 2)-(1-\varepsilon)(n / 2)>(\varepsilon / 4) n
$$

By the Claim and $\varepsilon_{1} \leq \varepsilon^{3} / 10^{3}$, we know that the number of $\left\{y_{1}, y_{2}, y_{3}\right\} \in\left(\begin{array}{c}Y^{\prime} \\ 3\end{array}\right)$ with $a y_{1} y_{2} y_{3} \in$ $\mathcal{H}$ is at least

$$
\left(\begin{array}{c}
\left|Y^{\prime}\right| \\
3
\end{array}\right)-d_{M}(a)>\left(\begin{array}{c}
(\varepsilon / 4) n \\
3
\end{array}\right)-\varepsilon_{1} n^{3}>2 \varepsilon_{1} n^{3}-\varepsilon_{1} n^{3}=\varepsilon_{1} n^{3}
$$

Each such $\left\{y_{1}, y_{2}, y_{3}\right\} \in\left(\begin{array}{c}Y^{\prime} \\ 3\end{array}\right)$ yields the $P_{4}$ given by

$$
b c d y_{1}, \quad b c d y_{2}, \quad b c d y_{3}, \quad e, \quad a y_{1} y_{2} y_{3} .
$$

We have argued above that for each $e \in B_{3}$ there are at least $\varepsilon_{1} n^{3}$ copies of $P_{4}$ containing $e$. Each such copy of $P_{4}$ contains a unique edge of $B_{3}$. Consequently, we obtain

$$
\# P_{4} \geq\left|B_{3}\right|\left(\varepsilon_{1} n^{3}\right)>(\varepsilon / 2)|B|\left(\varepsilon_{1} n^{3}\right)>\left(\varepsilon^{2} \varepsilon_{1} / 8\right) n^{4}>\delta n^{4} .
$$

This contradiction completes the proof of the theorem.

\subsection{Counting Expanded triangles}

Recall that $c\left(n, C_{3}\right)=3(n / 2)^{2}+\Theta(n)$. Theorem 10 follows from the following result.

Theorem 28. For every $\varepsilon>0$ there exists $\delta>0$ and $n_{0}$ such that the following holds for $n>n_{0}$. Every $n$ vertex 4 -graph with $b^{4}(n)+1$ edges contains either

- an edge that lies in at least $(3-\varepsilon)(n / 2)^{2}$ copies of $C_{3}$, or

- at least $\delta n^{4}$ copies of $C_{3}$.

Proof of Theorem 10. Remove $q-1$ edges from $\mathcal{H}$ and apply Theorem 28 . If we find $\delta n^{4}$ copies of $C_{3}$, then since $q<\delta n^{2}$, the number of copies is much larger than $q(1-\varepsilon) c\left(n, C_{3}\right)$ and we are done. Consequently, we find an edge $e_{1}$ in at least $(3-\varepsilon)(n / 2)^{2}>(1-\varepsilon) c\left(n, C_{3}\right)$ copies of $C_{3}$. Now remove $q-2$ edges from $\mathcal{H}-e_{1}$ and repeat this argument to obtain $e_{2}$. In this way we obtain edges $e_{1}, \ldots, e_{q}$ as required. 
Sharpness follows by the following construction: Add a collection of $q$ 4-tuples to $B^{4}(n)$ within one of the parts (say $X$ ) such that every two 4-tuples have at most one point in common. It is well-known that such quadruple-systems exist of size $\delta n^{2}$ (in fact such Steiner systems also exist for an appropriate congruence class of $n$ ). It is easy to see that each added 4-tuple lies in at most $3(n / 2)^{2}$ copies of $C_{3}$, since there are three ways to partition the edge into two disjoint pairs, and for each of these ways, there are at most $(n / 2)^{2}$ copies of $C_{3}$ using this partition. Moreover, no two added edges lie in a copy of $C_{3}$ since they share at most one point. Consequently, the number of copies of $C_{3}$ is at most $3 q(n / 2)^{2}$.

We need the following stability result proved by Keevash and Sudakov [15].

Theorem 29. ( $C_{3}$ stability [15]) Let $\mathcal{H}$ be a 4-graph with $n$ vertices and $b^{4}(n)-o\left(n^{4}\right)$ edges that contains no copy of $C_{3}$. Then there is a partition of the vertex set of $\mathcal{H}$ into $X \cup Y$ so that the number of edges that intersect $X$ or $Y$ in an even number of points is o( $\left.n^{4}\right)$. In other words, $\mathcal{H}$ can be obtained from $B^{4}(n)$ by adding and deleting a set of o $\left(n^{4}\right)$ edges.

Proof of Theorem 28. Given $\varepsilon$ let $0<\delta \ll \varepsilon$. Write $o_{\delta}(1)$ for any function that approaches zero as $\delta$ approaches zero and moreover, $o_{\delta}(1) \ll \varepsilon$. Let $n$ be sufficiently large and let $\mathcal{H}$ be an $n$ vertex 4 -graph with $b^{4}(n)+1$ edges. Write $\# C_{3}$ for the number of copies of $C_{3}$ in $\mathcal{H}$.

As in the proof of Theorem 22 (just replacing $3 / 32$ by $1 / 2$ ), we may assume that $\mathcal{H}$ has minimum degree at least $d=\left(1 / 2-o_{\delta}(1)\right)\left(\begin{array}{l}n \\ 3\end{array}\right)$.

If $\# C_{3} \geq \delta n^{4}$, then we are done so assume that $\# C_{3}<\delta n^{4}=\left(\delta / n^{2}\right) n^{6}$. Then by the Removal lemma, there is a set of at most $\delta n^{4}$ edges of $\mathcal{H}$ whose removal results in a 4 -graph $\mathcal{H}^{\prime}$ with no copies of $C_{3}$. Since $\left|\mathcal{H}^{\prime}\right|>b^{4}(n)-\delta n^{4}$, by Theorem 29, we conclude that there is a partition of $\mathcal{H}^{\prime}$ (and also of $\mathcal{H}$ ) into two parts such that the number of edges intersecting some part in an even number of points is $o_{\delta}\left(n^{4}\right)$. Now pick a partition $X \cup Y$ of $\mathcal{H}$ that maximizes $e(X, Y)$, the number of edges that intersect each part in an odd number of points. We know that $e(X, Y) \geq b^{4}(n)-o_{\delta}\left(n^{4}\right)$, and an easy calculation also shows that each of $X, Y$ has size $n / 2 \pm o_{\delta}(n)$.

Let $B$ be the set of edges of $\mathcal{H}$ that intersect one (and therefore both) of $X, Y$ in an even number of points. Let $G=\mathcal{H}-B$ be the set of edges of $\mathcal{H}$ that intersect both $X, Y$ in an odd number of points. Let $M$ be the set of 4-tuples which intersect both parts in an odd number of points and are not edges of $\mathcal{H}$. Then $\mathcal{H}-B \cup M=G \cup M$ is an odd 4-graph 
with partition $X, Y$, so it has at most $b^{4}(n)$ edges. We conclude that

$$
|M|<|B|<o_{\delta}\left(n^{4}\right)
$$

in particular $B \neq \emptyset$.

Given vertices $a, b$ and hypergraph $F$, write $d_{F}(a b)$ for the number of edges of $F$ containing both $a$ and $b$. The rest of the proof has many similarities (modulo technical changes) to the proof of the exact Turán result for $C_{3}$ in [15]. Let $\varepsilon_{1}=\varepsilon / 10^{5}$.

Claim 1. For every two vertices $a, b \in V:=X \cup Y$, either $d_{G}(a b)<\varepsilon_{1} n^{2}$ or $d_{B}(a b)<\varepsilon_{1} n^{2}$. Proof. Suppose, for contradiction, that both $d_{G}(a b)$ and $d_{B}(a b)$ are at least $\varepsilon_{1} n^{2}$. Pick $e \in B$ and $f \in G$ with $e \cap f=\{a, b\}$. Note that in all cases $g_{e, f}=e \cup f-\{a, b\} \in M \cup G$, i.e., $g_{e, f}$ intersects both parts in an odd number of points. The number of such pairs $e, f$ is at least $\left(\varepsilon_{1} n^{2}\right)^{2} / 2>2 \delta n^{4}$ (the factor of 2 is to ensure that $e \cap f=\{a, b\}$ ). If at least $\delta n^{4}$ of these pairs form a copy of $C_{3}$, then we are done, so we may assume that at least $\left(\varepsilon_{1} n^{2}\right)^{2} / 4$ of these pairs satisfy $g_{e, f} \in M$. This contradicts (12) and completes the proof of the Claim.

Claim 2. $d_{B}(v)<\left(\varepsilon / 10^{3}\right) n^{3}$ for every $v \in V$.

Proof. Let us fix a vertex $v \in V, \varepsilon^{\prime}=\varepsilon / 10^{3}>24 \varepsilon_{1}$, and assume for contradiction that $d_{B}(v) \geq \varepsilon^{\prime} n^{3}$. Call vertex $w \in V-\{v\} \operatorname{good}$ if $d_{B}(v w)<\varepsilon_{1} n^{2}$, otherwise say that $w$ is bad. Claim 1 implies that if $w$ is bad, then $d_{G}(v w)<\varepsilon_{1} n^{2}$. Moreover, the number of bad vertices is at least $\varepsilon^{\prime} n$ for otherwise we obtain the contradiction

$$
d_{B}(v) \leq \sum_{w b a d} d_{B}(v w)+\sum_{w g o o d} d_{B}(v w)<\varepsilon^{\prime} n\left(\begin{array}{l}
n \\
2
\end{array}\right)+n \varepsilon_{1} n^{2}<\varepsilon^{\prime} n^{3} .
$$

Next we observe that $d_{G}(v) \geq d_{B}(v)$ for otherwise we could move $v$ to the other part and contradict the choice of $X, Y$. This implies that $d_{G}(v) \geq\left(1 / 4-o_{\delta}(1)\right)\left(\begin{array}{l}n \\ 3\end{array}\right)$. If the number of good vertices is less than $n / 18$, then

$$
d_{G}(v) \leq \sum_{w \text { good }} d_{G}(v w)+\sum_{w b a d} d_{G}(v w)<\frac{n}{18}\left(\begin{array}{l}
n \\
2
\end{array}\right)+n\left(\varepsilon_{1} n^{2}\right)<\left(\frac{1}{6}+7 \varepsilon_{1}\right)\left(\begin{array}{l}
n \\
3
\end{array}\right) .
$$

This contradicts the lower bound on $d_{G}(v)$. We may therefore assume that the number of good vertices is $\alpha n$, where

$$
1 / 18 \leq \alpha \leq 1-\varepsilon^{\prime}
$$


Write $d_{\mathcal{H}}(v)=d_{G}(v)+d_{B}(v)$ and let us estimate these two terms separately. The number of edges of $G$ containing $v$ and a bad vertex is at most $((1-\alpha) n+1) \varepsilon_{1} n^{2}<\varepsilon_{1} n^{3}$. The number of edges of $G$ containing $v$ and no bad vertex is at most

$$
\frac{\alpha n(\alpha n-1)}{6}\left(\frac{1}{2}+o_{\delta}(1)\right) n \leq\left(\frac{\alpha^{2}}{2}+o_{\delta}(1)\right)\left(\begin{array}{l}
n \\
3
\end{array}\right) .
$$

The bound above is obtained by picking two good vertices which then restricts the edge being counted to one of the parts. This procedure counts each edge six times. We conclude that $d_{G}(v)<\left(\alpha^{2} / 2+6 \varepsilon_{1}\right)\left(\begin{array}{l}n \\ 3\end{array}\right)$.

The number of edges of $B$ containing $v$ and a good vertex is at most $\varepsilon_{1} n^{3}$. Using a similar argument to that used above, the number of edges of $B$ containing $v$ and no good vertex is at most $\left((1-\alpha)^{2} / 2+6 \varepsilon_{1}\right)\left(\begin{array}{l}n \\ 3\end{array}\right)$. We conclude that

$$
d_{\mathcal{H}}(v) \leq\left(\frac{\alpha^{2}+(1-\alpha)^{2}}{2}+12 \varepsilon_{1}\right)\left(\begin{array}{l}
n \\
3
\end{array}\right) .
$$

Using (13) and $\varepsilon_{1}<\varepsilon^{\prime} / 24$, we observe that

$$
\frac{\alpha^{2}+(1-\alpha)^{2}}{2}+12 \varepsilon_{1}=\frac{1}{2}+\alpha^{2}-\alpha+12 \varepsilon_{1}<\frac{1}{2}+\left(1-\varepsilon^{\prime}\right)^{2}-\left(1-\varepsilon^{\prime}\right)+12 \varepsilon_{1}<\frac{1}{2}-\frac{\varepsilon^{\prime}}{2} .
$$

Consequently, $d_{\mathcal{H}}(v)<\left(1 / 2-\varepsilon^{\prime} / 2\right)\left(\begin{array}{l}n \\ 3\end{array}\right)$. This contradicts the fact that $d_{\mathcal{H}}(v) \geq(1 / 2-$ $\left.o_{\delta}(1)\right)\left(\begin{array}{l}n \\ 3\end{array}\right)$ and completes the proof of the Claim.

The rest of the proof is devoted to showing that $d_{B}(v) \geq\left(\varepsilon / 10^{3}\right) n^{3}$ for some vertex $v$ and this contradicts Claim 2. Note that for every edge $e \in B$, there are at least $\left(3-o_{\delta}(1)\right)(n / 2)^{2}$ copies of $C_{3}$ containing $e$ where the other two edges in the copy are in $G$. Indeed, this is why $c\left(n, C_{3}\right)=(3+o(1))(n / 2)^{2}$. This requires some case analysis, for example, if $e=$ $\{a, b, c, d\} \subset X$, then for every choice of $(x, y) \in(X-e) \times Y$, and for every partition of $e$ into two disjoint pairs $p, q$, the three edges $e, p \cup\{x, y\}, q \cup\{x, y\}$ form a copy of $C_{3}$ and $p \cup\{x, y\}, q \cup\{x, y\} \in G$. The number of such copies is therefore the number of $(x, y)$ times the number of pairs $p, q$ and this is $\left(3-o_{\delta}(1)\right)(n / 2)^{2}$. The case $a, b \in X, c, d \in Y$ is similar except that the argument further breaks into two cases depending on the choice of $p, q$. We omit these details.

Claim 3. There is a pair of vertices $a, b$ with $d_{B}(a b)>(\varepsilon / 48) n^{2}$

Proof. For every $e \in B$, at least $(\varepsilon / 2)(n / 2)^{2}$ of the copies of $C_{3}$ using $e, f, g$ with $f, g \in G \cup M$ must have at $f \in M$ or $g \in M$. Otherwise, there are at least $\left(3-o_{\delta}(1)-\varepsilon / 2\right)(n / 2)^{2}>$ 
$(3-\varepsilon)(n / 2)^{2}$ copies of $C_{3}$ containing $e$ and we are done. The edge in $M$ is counted precisely once, since a copy of $C_{3}$ is uniquely determined by two of its edges. We conclude that for each $e \in B$, there are at least $(\varepsilon / 2)(n / 2)^{2}$ edges of $M$ that intersect $e$ is exactly two points. Now form a bipartite graph with parts $B, M$ where $e \in B$ is adjacent to $f \in M$ if $|e \cap f|=2$. Since $|M|<|B|$, and each $e \in B$ is adjacent to at least $(\varepsilon / 2)(n / 2)^{2}$ different $f \in M$, we conclude that there exists $f \in M$ that is adjacent to more than $(\varepsilon / 2)(n / 2)^{2}$ different $e \in B$. At least $1 / 6$ of these $e$ intersect $f$ in the same pair of points $a, b$. Consequently, $d_{B}(a b)>(\varepsilon / 12)(n / 2)^{2}=(\varepsilon / 48) n^{2}$ and the Claim is proved.

Let us fix $a, b$ from Claim 3. For each edge $e=a b c d \in B$, there are at least $n^{2} / 5$ pairs $r, s \in V$ such that the three sets e, acrs, bdrs form a copy of $C_{3}$. By Claim 3, the number of such potential copies of $C_{3}$ is at least $(\varepsilon / 240) n^{4}>2 \delta n^{4}$, so for at least half of them, either acr $\in M$ or bdrs $\in M$. Each such 4-tuple of $M$ is counted at most $n$ times, since $a, b, r, s$ are fixed. This gives us at least $(\varepsilon / 480) n^{3} 4$-tuples of $M$ containing either $a$ or $b$. At least $(\varepsilon / 960) n^{3}$ must contain the same point, say $a$. Consequently, $d_{M}(a) \geq(\varepsilon / 960) n^{3}$. We know that $d_{\mathcal{H}}(a) \geq\left(1 / 2-o_{\delta}(1)\right)\left(\begin{array}{l}n \\ 3\end{array}\right)$, and the above argument shows that $d_{G}(a) \leq$ $\left(1 / 2+o_{\delta}(1)-\varepsilon / 960\right)\left(\begin{array}{l}n \\ 3\end{array}\right)$. We conclude that $d_{B}(a)>\left(\varepsilon / 10^{3}\right) n^{3}$ which contradicts Claim 2 and completes the proof. 